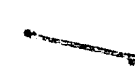

WSRC-TR--92-397

DE93 006565

SECOND QUARTER 1992

\title{
METALLURGICAL LABORATORY HAZARDOUS WASTE MANAGEMENT FACILITY GROUNDWATER MONITORING REPORT (U)
}

KEY WORDS

AMB wells tetrachloroethylene total alpha-emitting radium total organic halogens trichloroethylene

PUBLICATION DATE: SEPTEMBER 1992

Authorized Derivative Classifier:

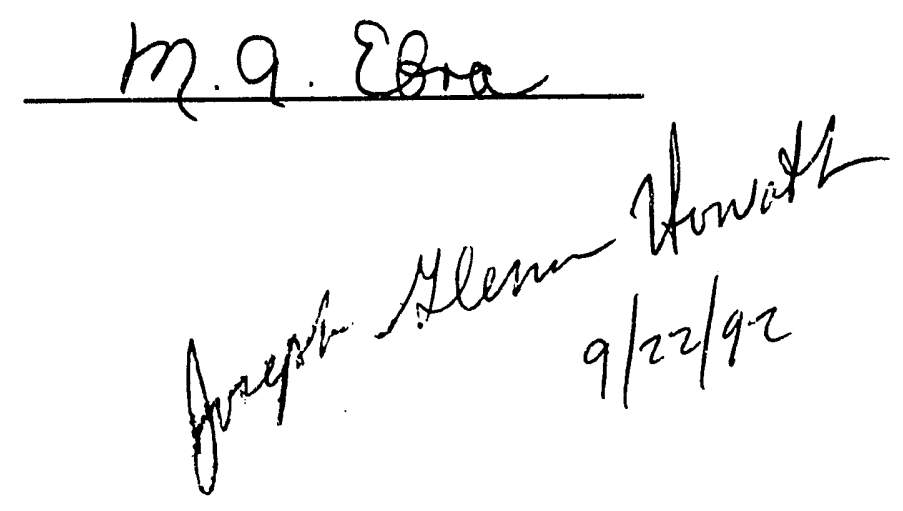


All copies of thi groundwater quality assessment report transmitted to the regulatory agencies are stamped by a registered professional geologist (P.G.) of South Carolina. Copies of the report retained at the Savannah River Site for reference do not include the registered P.G. stamp. 


\section{Abstract}

During second quarter 1992, 18 groundwater monitoring wells of the AMB series at the Metallurgical Laboratory Hazardous Waste Management Facility (Met Lab HWMF) were visited for sampling. Groundwater samples were analyzed for certain heavy metals, indicator parameters, radionuclides, volatile organic compounds, and other constituents. This report describes the results that exceeded the U.S. Environmental Protection Agency Primary Drinking Water Standards (PDWS) and the Savannah River Site flagging criteria during the quarter.

Tetrachloroethylene exceeded the PDWS in wells AMB 4A, 4D, 5, and 7A; trichloroethylene was elevated in wells $\mathrm{AMB} 4 \mathrm{~A}, 4 \mathrm{~B}, 4 \mathrm{D}, 5$, and $7 \mathrm{~A}$; and total alpha-emitting radium (radium224 and radium-226) activity exceeded the PDWS in well AMB 5 . Total organic halogens exceeded the Flag 2 criterion in wells AMB 4A, 4D, 5, 7A, and 7B; manganese exceeded the Flag 2 criterion in well AMB 4D; iron exceeded the Flag 2 criterion in well AMB 10DD; and $\mathrm{pH}$ exceeded the Flag 2 criterion in well AMB 10A.

Elevated constituents were not found in the upgradient water-table wells. However, downgradient well AMB 4D contzined elevated levels of manganese, tetrachloroethylene, total organic halogens, and trichloroethylene; and downgradient well AMB 5 contained elevated levels of tetrachloroethylene, total organic halogens, total alpha-emitting radium, and trichloroethylene. Downgradient Congaree wells did not contain elevated constituents. 


\section{Contents}

Page

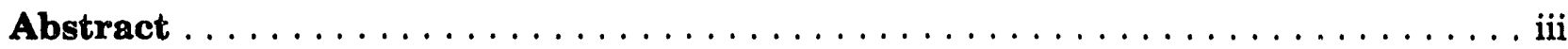

List of Figures $\ldots \ldots \ldots \ldots \ldots \ldots \ldots \ldots \ldots \ldots \ldots \ldots \ldots \ldots \ldots \ldots$

List of Tables $\ldots \ldots \ldots \ldots \ldots \ldots \ldots \ldots \ldots \ldots \ldots \ldots \ldots \ldots \ldots$

Executive $\operatorname{Summary} \ldots \ldots \ldots \ldots \ldots \ldots \ldots \ldots \ldots \ldots \ldots \ldots$

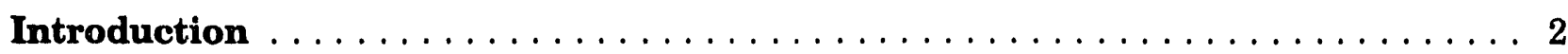

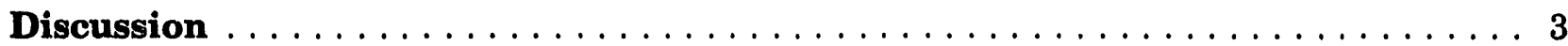

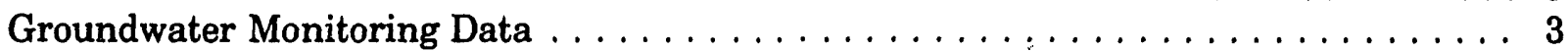

Groundwater Monitoring Results $\ldots \ldots \ldots \ldots \ldots \ldots \ldots \ldots \ldots \ldots$

Time Series Plots . . . . . . . . . . . . . . . . . . . . . . . . . . 4

Groundwater Gradient, Flow Direction, and Flow Rates $\ldots \ldots \ldots \ldots \ldots \ldots \ldots \ldots$

Results for Upgradient vs. Downgradient Wells $\ldots \ldots \ldots \ldots \ldots \ldots \ldots \ldots \ldots \ldots \ldots$

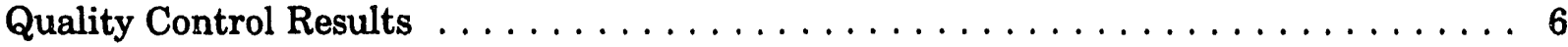

Conclusions $\ldots \ldots \ldots \ldots \ldots \ldots \ldots \ldots \ldots \ldots \ldots \ldots \ldots \ldots \ldots \ldots \ldots$

Errata $\ldots \ldots \ldots \ldots \ldots \ldots \ldots \ldots \ldots \ldots \ldots \ldots \ldots \ldots \ldots \ldots \ldots \ldots$

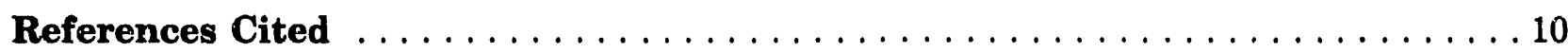

Appendix A-Primary Drinking Water Standards $\ldots \ldots \ldots \ldots \ldots \ldots \ldots$

Appendix B-Flagging Criteria $\ldots \ldots \ldots \ldots \ldots \ldots \ldots \ldots \ldots \ldots \ldots \ldots$

Appendix C-Figures $\ldots \ldots \ldots \ldots \ldots \ldots \ldots \ldots \ldots \ldots \ldots \ldots \ldots \ldots \ldots \ldots$

Appendix D-Groundwater Monitoring Results Tables $\ldots \ldots \ldots \ldots \ldots \ldots$ D-1

Appendix E-Time Series Plots $\ldots \ldots \ldots \ldots \ldots \ldots \ldots \ldots \ldots \ldots \ldots \ldots$

Appendix F-Hydrographs $\ldots \ldots \ldots \ldots \ldots \ldots \ldots \ldots \ldots \ldots \ldots \ldots \ldots$

Appendix G-Water-Elevation Contour Maps $\ldots \ldots \ldots \ldots \ldots \ldots \ldots \ldots$ G-1 


\section{List of Figures}

Page

1. Location of the Metallurgical Laboratory HWMF at the Savannah River Site. . . . . C-2

2. Location of the Metallurgical Laboratory HWMF in A and M Areas. . . . . . . . . C-3

3. Water-Elevation Contour Map of the Water Table at the Metallurgical Laboratory HWMF and Locations of the Water-Table Wells. . . . . . . . . . . . . . . C-4

4. Potentiometric Surface Map of the Upper Section of the Congaree Aquifer at the Metallurgical Laboratory HWMF and Locations of Wells that Monitor the Upper Sectioni of the Congaree $\ldots \ldots \ldots \ldots \ldots \ldots \ldots \ldots \ldots \ldots$

5. Potentiometric Surface Map of the Lower Section of the Congaree Aquifer at the Metallurgical Laboratory HWMF and Locations of Wells that Monitor the Lower Section of the Congaree $\ldots \ldots \ldots \ldots \ldots \ldots \ldots \ldots \ldots$

6. Water-Elevation Contour Map of the Water Table in A and M Areas . . . . . . . . C-7

\section{List of Tables}

1. Constituents Exceeding the Primary Drinking Water Standards $\ldots \ldots \ldots \ldots$ D-3

2. Constituents Exceeding Half the Primary Drinking Water Standards

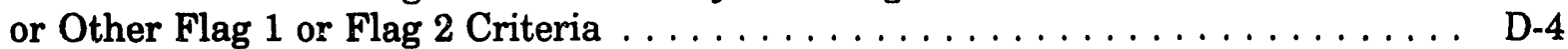

3. Groundwater Monitoring Results for Individual Wells $\ldots \ldots \ldots \ldots \ldots \ldots \ldots$ 


\section{Executive Summary}

The monitoring wells at the Metallurgical Laboratory Hazardous Waste Management Facility (Met Lab HWMF) are sampled quarterly as part of the Savannah River Site (SRS) Groundwater Monitoring Program to comply with South Carolina Hazardous Waste Management Regulations.

During second quarter 1992,18 wells were monitored at the facility. Nine wells monitor the water-table uni (wells AMB 4D, 5, 6, 7, 8D, 9D, 10D, 11D, and 12D); eight wells (AMB 4A, $4 \mathrm{~B}, 7 \mathrm{~A}, 7 \mathrm{~B}, 10 \mathrm{~A}, 10 \mathrm{~B}, 11 \mathrm{~B}$, and 13AR) monitor the upper and lower sections of the Congaree aquifer; and well AMB 10DD monitors a perched groundwater zone beneath the facility. Samples from these wells were analyzed for alkalinity, herbicides, major ions, pesticides, $\mathrm{pH}$, radionuclides, specific conductance, total dissolved solids, total organic carbon, total organic halogens, toxic metals, volatile organic compounds, and other constituents. This report describes the results that exceeded the U.S. Environmental Protection Agency Primary Drinking Water Standards (PDWS) and the SRS flagging criteria.

Tetrachloroethylene exceeded the PDWS in wells AMB 4A, 4D, 5, and 7A; and trichloroethylene was elevated in wells AMB 4A, 4B, 4D, 5, and 7A. The highest concentrations of tetrachloroethylene and trichloroethylene appeared in well ALB $4 \mathrm{~A}$, located in the lower part of the Congaree, at $44 \mu \mathrm{g} / \mathrm{L}$ and $812 \mu \mathrm{g} / \mathrm{L}$, respectively. Total alpha-emitting radium (radium-224 and radium-226) activity exceeded the PDWS in well $\mathrm{AMB} 5$ at $6.3 \mathrm{E}+00 \mathrm{pCi} / \mathrm{L}$.

Total organic halogens exceeded the Flag 2 criterion in wells AMB 4A, 4D, 5, 7A, and 7B, with a maximum value of $174 \mu \mathrm{g} / \mathrm{L}$ in well AMB 7A. Manganese exceeded the Flag 2 criterion in well AMB $4 \mathrm{D}$ at $60 \mu \mathrm{g} / \mathrm{L}$; iron exceeded the Flag 2 criterion in well AMB $10 \mathrm{DD}$ at $5,840 \mu \mathrm{g} / \mathrm{L}$; and $\mathrm{pH}$ exceeded the Flag 2 alkaline criterion in well AMB $10 \mathrm{~A}$ at $12 \mathrm{pH}$ units.

Elevated constituents were not found in the upgradient water-table wells AMB $11 \mathrm{D}$ and 12D or in downgradient water-table wells $\mathrm{AMB} 6,7,8 \mathrm{D}, 9 \mathrm{D}$, and 10D. However, downgradient water-table well AMB $4 \mathrm{D}$ contained elevated levels of manganese, tetrachloroethylene, total organic halogens, and trichloroethylene; and downgradient water-table well AMB 5 contained elevated levels of tetrachloroethylene, total organic halogens, total alpha-emitting radium, and trichloroethylene. Downgradient Congaree wells did not contain elevated constituents.

The estimate of the flow rate for groundwater in the water-table unit beneath the Met Lab HWMF is $0.4 \mathrm{ft} /$ day or $150 \mathrm{ft} /$ year, and the flow rate estimate for groundwater in the upper section of the Congaree is $0.01 \mathrm{ft} /$ day or $3.7 \mathrm{ft} /$ year. The horizontal gradient in the lower section of the Congaree was too low to calculate reliably the flow rate in this hydrostratigraphic unit. 


\section{Introduction}

The Metallurgical Laboratory Hazardous Waste Management Facility (Met Lab HWMF) is located in the eastern portion of A Area at the Savannah River Site (SRS). The facility consists of the process sewer line leading to the Metallurgical Laboratory Basin, the Metallurgical Laboratory Basin, the drainage outfall to the nearby Carolina bay, and the Carolina bay. The basin received waste water effluent from the Metallurgical Laboratory Building from 1956 until 1985. Waste water released to the basin consisted of laboratory wastes from metallographic sample preparation and corrosion testing of stainless steel and nickel-based alloys. The quantity of waste water discharged to the basin was small, averaging approximately 1,000 gallons/day (Heffner and Exploration Resources, 1991).

The Met Lab HWMF was named in Section III of the Natural Resources Defense Council et al. Consent Decree (May 26, 1988) and is subject to the requirements of Subtitle C of the Resource Conservation and Recovery Act (RCRA). A quarterly report describing the results of the assessment monitoring at the Met Lab HWMF is required to meet South Carolina Hazardous Waste Management Regulations (R.61-79.265, Subpart F, Groundwater Monitoring).

Eighteen wells monitor the water-table unit (wells AMB 4D, 5, 6, 7, 8D, 9D, 10D, 11D, and 12D), the upper portion of the Congaree aquifer (wells AMB 4B, 7B, 10B, and 11B), the lower portion of the Congsire aquifer (wells AMB 4A, 7A, 10A, and 13AR), and a perched groundwater zone (well AMB 10OD) beneath the Met Lab HWMF. These wells are sampled quarterly, and the results are reported to the South Carolina Department of Health and Environmental Control (SCDHEC). The wells that monitor the upper and lower sections of the Congaree aquifer were proposed in association with the RCRA Part B Post-Closure Care Permit Application and the Groundwater Quality Assessment Plan (Jerome, 1990) for the facility and were installed and monitored for the first time during third quarter 1991. 


\section{Discussion}

\section{Groundwater Monitoring Data}

Eighteen wells were visited for sampling at the Met Lab HWMF during second quarter 1992. The samples were analyzed for alkalinity, herbicides, major ions, pesticides, $\mathrm{pH}$, radionuclides, specific conductance, total dissolved solids, total organic carbon, total organic halogens, toxic metals, volatile organic compounds, and other constituents. The laboratory that conducted the analyses was certified by SCDHEC to analyze for these specific parameters. This report describes monitoring results that exceeded the U.S. Environmental Protection Agency Primary Drinking Water Standards (PDWS) (Appendix A) and the SRS flagging criteria (Appendix B).

In general, the flagging criteria established by the Environmental Monitoring Section of the Environmental Protection Department at SRS for identifying constituents in high concentrations in groundwater are as follows:

- Flag 2 criteria are based on the PDWS; for constituents that do not have a PDWS, Flag 2 criteria are based on the Secondary Drinking Water Standard (SDWS) or 10 times the method detection limit (MDL) as described in Appendix B. Constituent levels that equal or exceed Flag 2 criteria are described as elevated in this report.

- Flag 1 criteria are based on half the PDWS; for constituents that do not have a PDWS, Flag 1 criteria are based on half the SDWS or 5 times the MDL as described in Appendix B. Flag 1 levels may reflect analytical error; however, this level may indicate the initial detection of a contaminant. Thus, constituents exceeding Flag 1 criteria are included in this report and are described as slightly elevated.

- Flag 0 criteria are based on constituent levels below Flag 1 criteria or below the sample detection limits.

Illustrations of the monitored waste management unit at SRS (Figure 1), the location of the Met Lab HWMF within A and M areas (Figure 2), the flow directions of the groundwater beneath the facility (Figures 3, 4, and 5), and a water-elevation contour map of the water table in $A$ and $M$ areas (Figure 6) are in Appendix $C$. Monitoring results and analyses that exceeded holding times are presented in Appendix D; time series plots for $\mathrm{pH}$, specific conductance, tetrachloroethylene, total organic carbon, total organic halogens, total alpha-emitting radium (radium-224 and radium-226), and trichloroethylene are presented in Appendix E; hydrographs for the wells are in Appendix F; and large-scale water-elevation and potentiometric maps of the water-table and upper and lower sections of the Congaree hydrostratigraphic units are in Appendix G. 


\section{Groundwater Monitoring Results}

Results for analytes that exceeded the PDWS (see Appendix A) during second quarter 1992 are summarized in Table 1 (Appendix D). Tetrachloroethylene exceeded the PDWS in wells AMB 4A, 4D, 5, and 7A. The highest concentration of tetrachloroethylene, $44 \mu \mathrm{g} / \mathrm{L}$, appeared in well AMB $4 \mathrm{~A}$, which monitors the lower section of the Congaree. Trichloroethylene was elevated in wells AMB $4 \mathrm{~A}, 4 \mathrm{~B}, 4 \mathrm{D}, 5$, and $7 \mathrm{~A}$. The highest trichloroethylene value, $812 \mu \mathrm{g} / \mathrm{L}$, was found in well AMB 4A. Total alpha-emitting radium activity exceeded the PDWS in well $\mathrm{AMB} 5$ at $6.3 \mathrm{E}+00 \mathrm{pCi} / \mathrm{L}$.

Constituents that exceeded other Flag 2 criteria (see Appendix B) during second quarter 1392 are summarized in Table 2 (Appendix D). Total organic halogens exceeded the Flag 2 criterion in wells $A M B ~ 4 A, 4 D, 5,7 \AA$, and $7 B$, with a maximum value of $174 \mu \mathrm{g} / \mathrm{L}$ in well AMB 7A. Manganese exceeded the Flag 2 criterion in well AMB $4 \mathrm{D}$ at $60 \mu \mathrm{g} / \mathrm{L}$; iron exceeded the Flag 2 criterion in well AMB $10 \mathrm{DD}$ at $5,840 \mu \mathrm{g} / \mathrm{L}$; and $\mathrm{pH}$ exceeded the Flag 2 alkaline criterion in well AMB $10 \mathrm{~A}$ at $12 \mathrm{pH}$ units.

All results for individual wells are presented in Table 3 (Appendix D). Table 3 also indicates those analyses that exceeded holding times. Resampling of monitoring wells where sample holding times have been exceeded will be addressed by EPD/EMS.

The standard practice at SRS is to purge four we wlumes from each well and ensure that the field parameters have stabilized before sample callestion. A well that goes dry before purging is completed is allowed to recover and is sampled without purging within 24 hours. Table 3 (Appendix D) lists the amount of water purged from each well. During second quarter 1992, wells AMB 7 and 10A did not yield four well volumes prior to sampling. Well AMB 7 has a history of low yield and recovery. Well AMB 4 was abandoned September 30, 1991 , because it was close to the edge of the basin and would be covered by the cap during basin closure.

\section{Time Series Plots}

Time series plots of field $\mathrm{pH}$, field specific conductance, tetrachloroethylene, total organic carbon, total organic halogens, total alpha-emitting radium, and trichloroethylene were constructed for wells AMB 4A, 4B, 4D, 5, 6, 7, 7A, 7B, 8D, 9D, 10A, 10B, 10D, 10DD, 11B, 11D, 12D, and 13AR (Appendix E). Trends for these indicator parameters are as follows:

pH: Compared to the previous quarter, field pH values for wells $\mathrm{AMB} 5,6,7,7 \mathrm{~A}, 10 \mathrm{~B}$, $11 \mathrm{~B}$, and 12D decreased; values for wells AMB 4A, 4B, 4D, 8D, 9D, 10A, 10DD, 11D, and 13AR increased; and values for wells $A M B ~ 7 B$ and 10D did not change. The $\mathrm{pH}$ in well AMB 10A exceeded the SRS Flag 2 alkaline criterion at $\mathrm{pH} 12.6$. The values for the remaining wells ranged between approximately $\mathrm{pH} 5$ and 7 .

Specific conductance: Compared to the previous quarter, field specific conductance for wells AMB 4A, 4B, 4D, 5, 7, 7A, 9D, 10B, and 10D decreased; values for wells AMB 6, 7B, 8D, 10A, 10DD, 11B, 11D, 12D, and 13AR increased. Specific conductance in well AMB $10 \mathrm{~A}$ increased from $216 \mu \mathrm{S} / \mathrm{cm}$ to $2,290 \mu \mathrm{S} / \mathrm{cm}$; the latter value exceeds the SRS Flag 2 criterion. 
Tetrachloroethylene: Compared to the previous quarter, tetrachloroethylene concentrations in well AMB 7A decreased; concentrations in wells AMB 4A, 4B, and 4D increased; concentrations in well AMB 5 remained unchanged; and concentrations in the remaining wells remained at or below the MDL. Tetrachloroethylene concentrations in wells $A M B$ AA, 4D, 5, and 7A exceeded the PDWS.

Total alpha-emitting radium: Compared to the previous quarter, total alpha-emitting radium activities in wells $A M B$ DD and 7 decreased; activities in wells AMB 6, 9D, 10D, $11 B$, and 13AR increased; the activity in well AMB 5 remained unchanged; and activities in the remaining wells remained at or below the MDL. Total alpha-emitting radium activity in well AMB 5 exceednd the PDWS.

Total organic carbon: Compared to the previous quarter, total organic carbon concentrations in well AMB 10DD decreased; concentrations in wells AMB 8D and $10 \mathrm{~A}$ increased; and concentrations in the remaining wells remained at or below the MDL.

Total organic halogens: Compared to the previous quarter, total organic halogens in wells AMB 4A, 4B, 5, 6, 8D, 9D, 10A, 10D, 10DD, and 12D decreased; concentrations in wells AMB 4D, 7, 7A, 7B, and 11B increased; and concentrations in the remaining wells remained at or below the MDL. Concentrations of total organic halogens in well AMB 4A, 4 i, 7A, and 7B exceeded the SRS Flag 2 criterion.

Trichloroethylene: Compared to the previous quarter, trichloroethylene concentrations in wells AMB 7, 7A, and 13AR decreased; concentrations in wells AMB 4A, 4B, 4D, 5, and 6 increased; and concentrations in the remaining wells remained at or below the MDL. This constituent continued to exceed the PDWS in wells AMB 4A, 4B, 4D, 5, and 7A.

\section{Groundwater Gradient, Flow Direction, and Flow Rates}

The water-table unit beneath the Met Lab HWMF slopes to the northwest and has an average gradient of $\backslash .0039$. Water-elevation contours of the water-table unit and the groundwater flow direction beneath the facility are shown in Figure 3 (Appendix C), and hydrographs for the wells are presented in Appendix F. Historically, the horizontal groundwater flow in the upper and lower sections of the Congaree aquifer is to the south (Figures 4 and 5, Appendix C). During second quarter 1992, horizontal flow in the upper section of the Congaree was to the southwest; the horizontal gradient in the lower section of the Congaree was too low to calculate horizontal flow direction reliably. Flow directions in this report are presented according to SRS grid coordinates.

The groundwater flow rate beneath the Met Lab HWMF is estimated using the following equation:

$$
\text { Flow (ft/day) }=\frac{\text { Hydraulic Conductivity (ft/day) }}{\text { Porosity (unitless) }} \times \frac{d h(f t)}{d l(f t)}
$$

Hydraulic conductivity constants estimated for the water-table unit, the upper section of the Congaree, and the lower section of the Congaree are $27,1.05$, and $1.48 \mathrm{ft} /$ day, respectively (WSRC, 1991). The effective porosity values estimated for the water-table unit, the upper 
section of the Congaree, and the lower section of the Congaree are $20 \%, 30 \%$, and $30 \%$, respectively. The value $d h$ is the difference in head, and $d l$ is the iength of the flow path. Flow rate estimations vary depending on the vertical gradient between wells, the size of the area under consideration, and the number of data points. The values presented here are useful as order of magnitude estimations only.

The flow rate estimate for groundwater in the water-table unit beneath the Met Lab HWMF is as follows (see Figure 3, Appendix C):

$$
\begin{aligned}
& \frac{27}{0.20} \times \frac{1.5}{480}=0.4 \mathrm{ft} / \text { day } \\
& 0.4 \mathrm{ft} / \text { day } \times 365 \text { days }=150 \mathrm{ft} / \text { year }
\end{aligned}
$$

The flow rate estimate for groundwater in the upper section of the Congaree beneath the Met Lab HWMF is as follows (see Figure 4, Appendix C):

$$
\begin{aligned}
& \frac{1.05}{0.30} \times \frac{1}{330}=0.01 \mathrm{ft} / \text { day } \\
& 0.01 \mathrm{ft} / \text { day } \times 365 \text { days }=3.7 \mathrm{ft} / \mathrm{year}
\end{aligned}
$$

The flow rate estimate for groundwater in the lower section of the Congaree beneath the Met Lab HWMF was not calculated for second quarter 1992 because of the low horizontal gradient.

\section{Results for Upgradient vs. Downgradient Wells}

Wells AMB 11D and 12D are designated upgradient water-table wells, and wells AMB 4D, 5, $6,7,8 \mathrm{D}, 9 \mathrm{D}$, and $10 \mathrm{D}$ are designated downgradient water-table wells relative to the Met Lab HWMF. During second quarter 1992, no elevated constituents occurred in the upgradient wells or in downgradient wells AMB 6, 7, 8D, 9D, and 10D. Downgradient well AMB 4D contained elevated levels of manganese, tetrachloroethylene, total organic halogens, and trichloroethylene; downgradient well AMB 5 contained elevated levels of tetrachloroethylene, total organic halogens, total alçaki *emitting radium, and trichloroethylene.

No wells have been designated as upgradient wells in the Congaree. Well AMB 11B is the downgradient well in the upper section of the Congaree, and well 13AR is the downgradient well in the lower section of the Congaree. Neither downgradient well contained elevated constituents.

\section{Quality Control Results}

Replicate analysis was performed for well AMB 8D during second quarter 1992. The results are provided in Table 3, Appendix D. 


\section{Conclusions}

During second quarter 1992, tetrachloroethylene exceeded the PDWS in wells AMB 4A, 4D, 5, and 7A. The highest concentration of tetrachloroethylene appeared in well AMB $4 \mathrm{~A}$ at $\Delta 4 \mu \mathrm{g} / \mathrm{L}$. Trichloroethylene was elevated in wells AMB $4 \mathrm{~A}, 4 \mathrm{~B}, 4 \mathrm{D}, 5$, and $7 \mathrm{~A}$. The highest trichloroethylene value, $812 \mu \mathrm{g} / \mathrm{L}$, occurred in well AMB 4A. Total alpha-emitting radium activity exceeded the PDWS in well AMB 5 at $6.3 \mathrm{E}+00 \mathrm{pCi} / \mathrm{L}$.

Total organic halogens exceeded the Flag 2 criterion in wells AMB 4A, 4D, 5, 7A, and 7B, with a maximum value of $174 \mu \mathrm{g} / \mathrm{L}$ in well AMB 7A. Manganese exceeded the Flag 2 criterion in well AMB $4 \mathrm{D}$ at $60 \mu \mathrm{g} / \mathrm{L}$; iron exceeded the Flag 2 criterion in well AMB $10 \mathrm{DD}$ at $5,840 \mu \mathrm{g} / \mathrm{L}$; and $\mathrm{pH}$ exceeded the Flag 2 alkaline criterion in well AMB $10 \mathrm{~A}$ at $12 \mathrm{pH}$ units.

Elevated constituents were not found in the upgradient water-table wells AMB $11 \mathrm{D}$ and $12 \mathrm{D}$ or in downgradient water-table wells AMB 6, 7, 8D, 9D, and 10D. However, downgradient water-table well AMB 4D contained elevated levels of manganese, tetrachloroethylene, total organic halogens, and trichloroethylene; and downgradient water-table well AMB 5 contained elevated levels of tetrachloroethylene, total organic halogens, total alpha-emitting radium, and trichloroethylene. Generally, constituents found in downgradient wells but not in upgradient wells at a waste management unit are considered products of the waste management unit. Downgradient Congaree wells did not contain elevated constituents.

The flow rate estimate for groundwater in the water-table unit beneath the Met Lab HWMF is $0.4 \mathrm{ft} /$ day or $150 \mathrm{ft} /$ year, and the flow rate estimate for groundwater in the upper section of the Congaree is $0.01 \mathrm{ft} /$ day or $3.7 \mathrm{ft} /$ year. These estimated groundwater flow rates represent the assumed maximum contaminant migration rates. The horizontal gradient in the lower section of the Congaree was too low to calculate reliably the flow rate in this hydrostratigraphic unit.

The RCRA Part B Post-Closure Care Permit Application for the Met Lab HWMF, submitted to SCDHEC on December 16, 1991, indicates that a small, isolated plume of trichloroethylene and tetrachloroethylene that exists in the water-table unit near the Met Lab HWMF is from the Met Lab basin (WSRC, 1991). The permit application also indicates that the primary source of the contamination in the deeper units near the Met Lab HWMF is the extensive organic halogens plume resulting from M-Area operations. SRS is addressing the effects of organic halogens near the Met Lab HWMF under the provisions of Section J, Volume III, of the SRS RCRA Part B Permit (M-Area HWMF Post-Closure Permit, Corrective Action Program) (WSRC, 1987).

The source of the total alpha-emitting radium is unknown; however, radium is a naturally occurring radionuclide at SRS, and its presence in groundwater may not necessarily be associated with waste management unit activities. 


\section{Errata}

Third Quarter 1991:

- Errors in the computer program used to mark analyses that exceed holding times caused a number of analyses to be incorrectly flagged as exceeding holding time.

- The only analyses performed by General Engineering on samples from the AMB well series during third quarter 1991 that exceeded holding times were the following:

Total organic halogen analyses for AMB 9D and 12D

Total phosphates (as P) for AMB 7A, 7B, 10B, and 13AR

Extraction for dioxins and furans for AMB 5, 6, 7, 8D, 9D, 10D, 10DD, and 12D

2-Chloroethyl vinyl ether for AMB 5, 6, 7, 8D, 9D, 10D, 10DD, 11D, 12D

(This analysis was not requested for any of these wells.)

Nitrate as nitrogen analysis for AMB 4, 6, and 7

Fifty-four Appendix IX analyses (all of the volatiles suite and chlorobenzilate from the BNAs) for AMB 9D (These were late by only one day and were reanalyzed due to surrogate failure on the first run.)

The error in the computer program was corrected prior to the fourth quarter 1991 reporting period.

Fourth Quarter 1991 and 1991 Summary:

- Page D-2, Holding Time: The statement concerning holding time for $\mathrm{pH}$ is incorrect. The statement should read "South Carolina Department of Health and Environmental Control allows only 15 minutes to elapse between sample collection and analysis for $\mathrm{pH}$. Thus, all laboratory $\mathrm{pH}$ analyses exceed holding time."

- The results of analyses performed using EPA Method 900.1 have been incorrectly referred to in the past as total radium results and have been inappropriately evaluated against the drinking water standard for combined radium-226 and radium-228. EPA Method 900.1 should be considered a gross radium alpha screening procedure; it may be used to screen drinking water for the necessity of performing a specific radium-226 analysis, but it gives no indication of the presence or quantity of radium-228 in the sample. This analysis will be referred to in the future as total alpha-emitting radium. 
First Quarter 1992:

- Page 6, Results for Upgradient vs. Downgradient Wells, paragraph 2, line 1: The first sentence is incorrect; the sentence should read, "No wells have been designated as upgradient wells in the Congaree."

- Page 7, paragraph 4, line 4: The sentence should read, "These estimated groundwater flow rates represent the assumed maximum contamination migration rates." 


\section{References Cited}

Heffner, J. D., and Exploration Resources, Inc., 1991. Technical Summary of Groundwater Quality Protection Program at the Savanrah River Site (1952-1986), Volume I-Site Geohydrology and Waste Sites, DPSP-88-1002. Westinghouse Savannah River Company, Aiken, SC.

Jerome, K. M., 1990. Groundwater Quality Assessment Plan for the Metallurgical Laboratory Hazardous Waste Management Facility, WSRC-RP-90-1185. Westinghouse Savannah River Company, Aiken, SC.

WSRC (Westinghouse Savannah River Company), 1987. Application for a PostClosure Permit, M-Area Hazardous Waste Management Facility, Volume III, Book 1, Rev. 2. Westinghouse Savannah River Company, Aiken, SC.

WSRC (Westinghouse Savannah River Company), 1991. Application for a Hazardous Waste Part B Post-Closure Care Permit, Rev. 1, Dec. 1991. Westinghouse Savannah River Company, Aiken, SC. 


\section{Primary Drinking Water Standards}

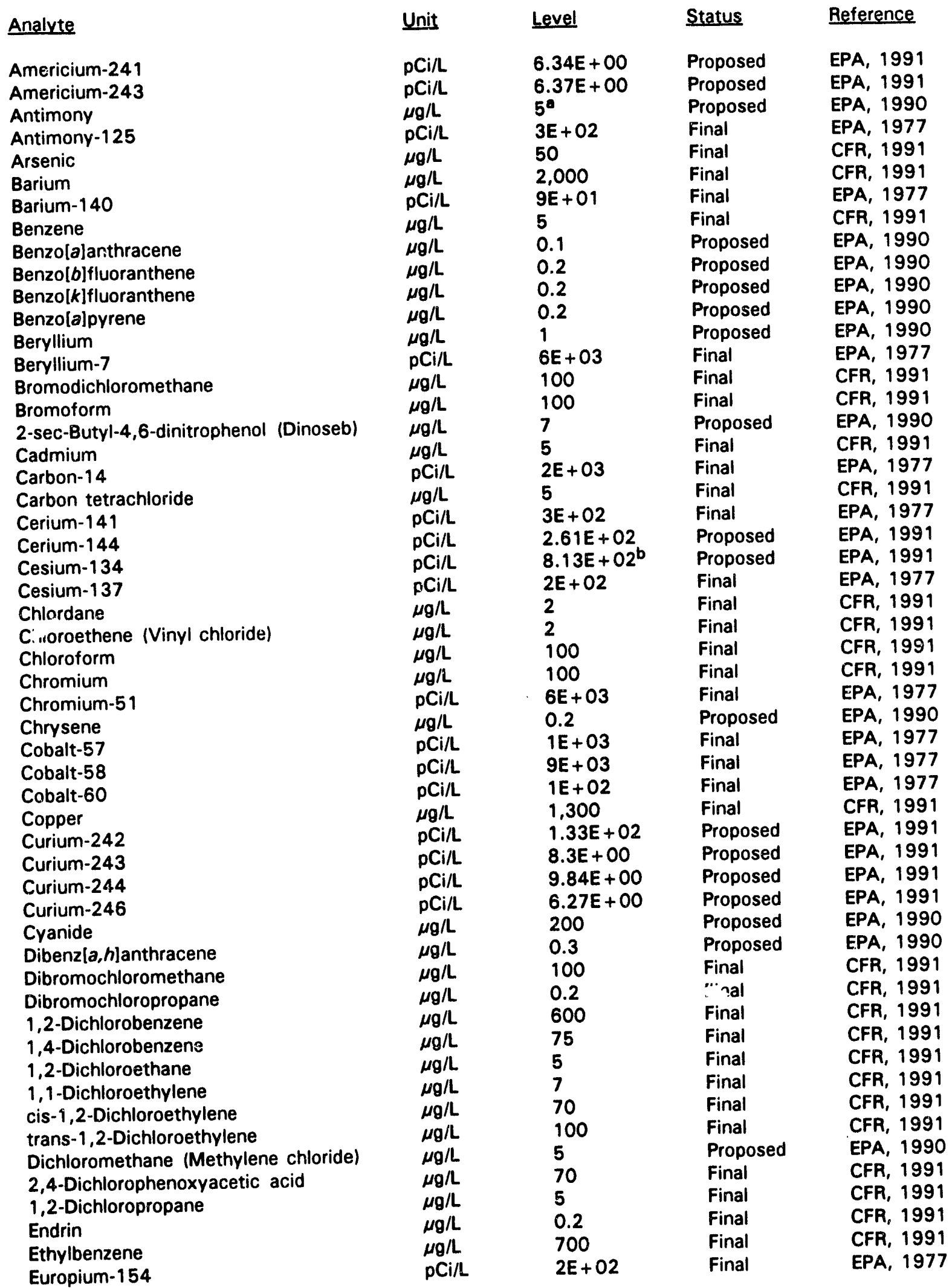




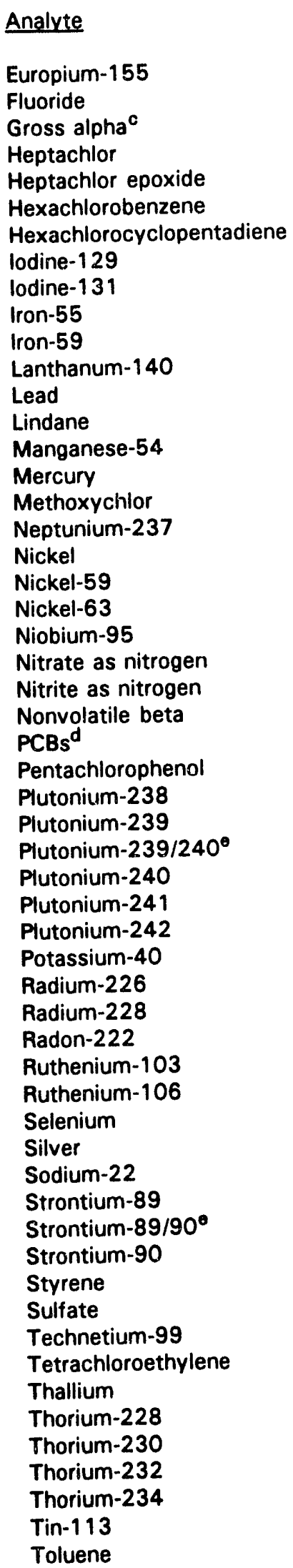

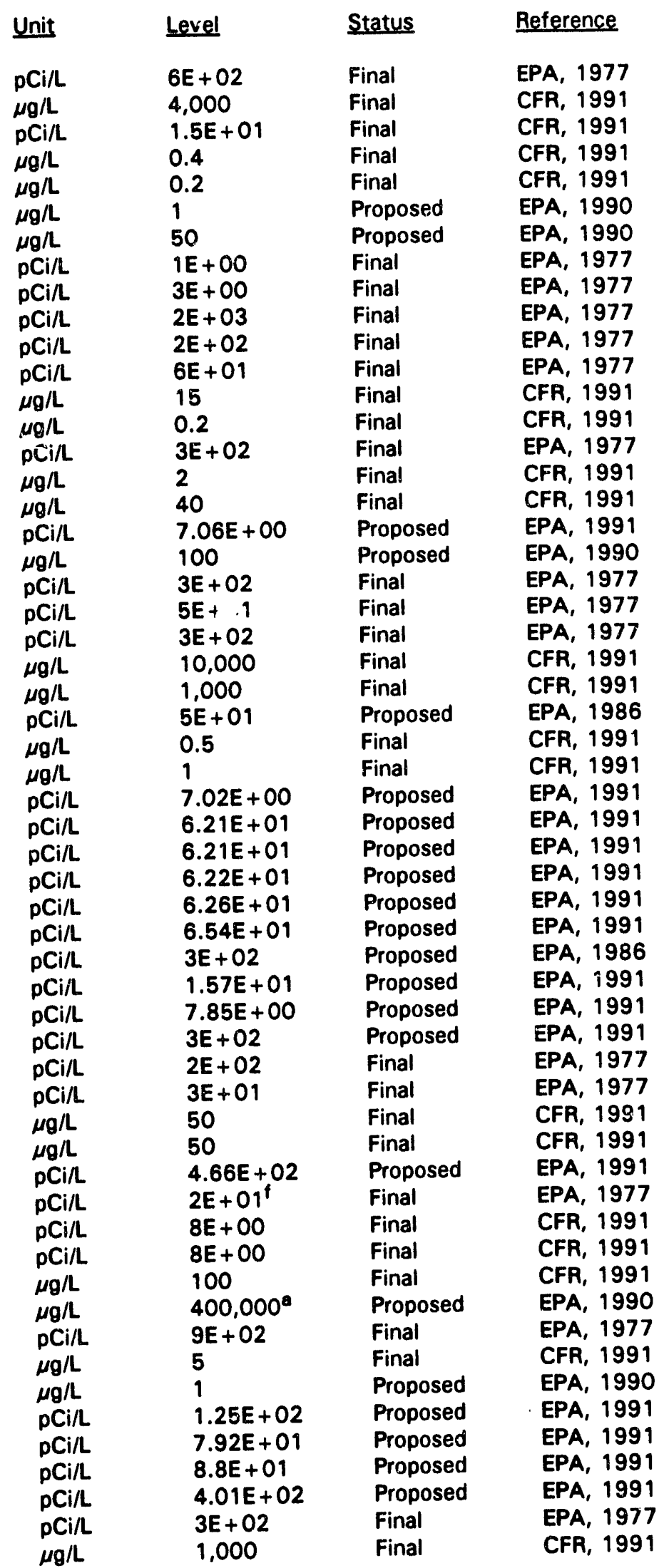




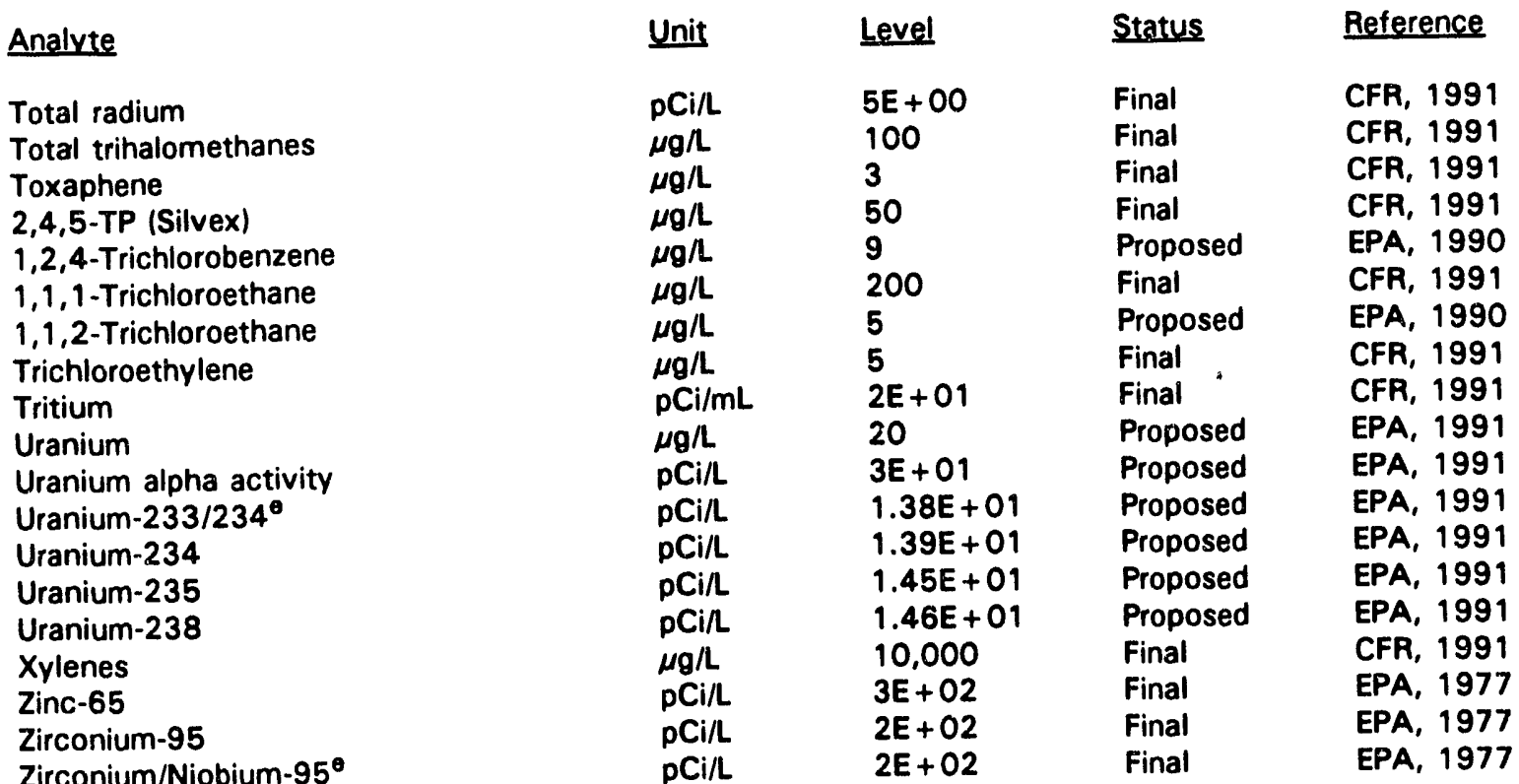

Note: Drinking water standards set by EPA (1977) correspond to the level at which each radionuclide contributes $4 \mathrm{mrem} / \mathrm{yr}$ of dose to an individual consuming $2 \mathrm{~L}$ of contaminated liquid a day. See EPA (1977) for details.

- This value is the lower of two proposed levels.

b EPD/EMS uses the proposed standard because it is a lower value; the final PDWS in 1977 may have been in error.

c The standard given is for gross alpha including radium-226 but excluding radon and uranium.

d Analyses were conducted in 1992 for the following: PCB 1016, PCB 1221, PCB 1232, PCB 1242, PCB 1248, PCB 1254, and PCB 1260.

- For double radionuclide analyses where each separate radionuclide has its own standard, the more stringent standard is used.

f This value is the lower of two levels given for strontium-89.

\section{References}

CFR (Code of Federal Regulations), 1991. National Primary Drinking Water Regulations, 40 CFR, Part 141, pp. 578-715. Washington, DC.

EPA (U.S. Environmental Protection Agency), 1977. National Interim Primary Drinking Water Regulations, EPA-570/9-76-003. Washington, DC.

EPA (U.S. Environmental Protection Agency), 1986. Water Pollution Control; National Primary Drinking Water Regulations, Radionuclides (Proposed), Federal Register, September 30, 1986, pp. 34835-34862. Washington, DC.

EPA (U.S. Environmental Protection Agency), 1990. National Primary and Secondary Drinking Water Regulations; Synthetic Organic Chemicals and Inorganic Chemicals; Proposed Rule, Federal Register, July 25, 1990, pp. 30369-30448. Washington, DC.

EPA (U.S. Environmental Protection Agency), 1991. National Primary Drinking Water Regulations; Radionuclides; Proposed Rule, Federal Register, July 18, 1991, pp. 33052-33127. Washington, DC. 


\section{- $\quad$ Appendix B - Flagging Criteria}




\section{Flagging Criteria}

Beginning in 1991, the Environmental Monitoring Section of the Environmental Protection Department (EPD/EMS) modified its guidelines for flagging constituents in the Groundwater Monitoring Program. These flagging cri eria are as follows:

- Flag 2 criteria for constituents equal the U.S. Environmental Protection Agency (EPA) Primary Drinking Water Standard, the EPA proposed Primary Drinking Water standard, or the EPA Secondary Drinking Water Standard. If a constituent does not have a drinking wuter standard, the Flag 2 criterion equals 10 times the method detection limit (MDL) calculated as the 90 th percentile detection limit obtained recently by one of the primary analytical laboratories.

- Flag 1 criteria for constituents equal one-half of the EPA Primary Drinking Water Standard, one-half the EPA proposed Primary Drinking Water Standard, or one-half the EPA Secondary Drinking Water Standard. If a constituent does not have an EPA drinking water standard, the Flag 1 criterion equals 5 times the MDL calculated as the 90 th percentile detection limit obtained recently by one of the primary analytical laboratories.

- Analytical results for constituents below Flag 1 or below sample detection limits are classified as Flag 0.

The following parameters are not assigned flagging criteria: alkalinity, calcium, carbonate, color, corrosivity, magnesium, odor, potassium, Eh, silica, sodium, total dissolved solids, total phosphorus, total phosphates (as P), and turbidity. In addition, common laboratory contaminants and cleaners including phthalates, methylene chloride, ketones, and toluene are not assigned flagging criteria.

Analyte
Acenaphthene
Acenaphthylene
Acetone
Acetonitrile (Methyl cyanide)
Acetophenone
2-Acetylaminofluorene
Acrolein
Acrylonitrile
Aldrin
Alkalinity (as $\mathrm{CaCO}_{3}$ )
Allyl chloride
Aluminum
Americium-241
Americium-243
$4-$ Aminobiphenyl
Ammonia
Ammonia nitrogen
Aniline
Anthracene
Antimony
Antimony-125

$\begin{array}{llll}\text { Unit } & & \text { Flag 1 } & \text { Flag 2 } \\ \mu \mathrm{g} / \mathrm{L} & 50 & 100 \\ \mu \mathrm{g} / \mathrm{L} & 50 & 100 \\ \mu \mathrm{g} / \mathrm{L} & 50 & 100 \\ \mu \mathrm{g} / \mathrm{L} & 500 & 1,000 \\ \mu \mathrm{g} / \mathrm{L} & 50 & 100 \\ \mu \mathrm{g} / \mathrm{L} & 50 & 100 \\ \mu \mathrm{g} / \mathrm{L} & 100 & 200 \\ \mu \mathrm{g} / \mathrm{L} & 100 & 200 \\ \mu \mathrm{g} / \mathrm{L} & 2.5 & 5 \\ & \mathrm{No} \mathrm{flag} & \mathrm{Noflag} \\ \mu \mathrm{g} / \mathrm{L} & 250 & 500 \\ \mu \mathrm{g} / \mathrm{L} & 100 & 200 \\ \mathrm{pCi} / \mathrm{L} & 3.17 \mathrm{E}+00 & 6.34 \mathrm{E}+00 \\ \mathrm{pCi} / \mathrm{L} & 3.19 \mathrm{E}+00 & 6.37 \mathrm{E}+00 \\ \mu \mathrm{g} / \mathrm{L} & 50 & 100 \\ \mu \mathrm{g} / \mathrm{L} & 500 & 1,000 \\ \mu \mathrm{g} / \mathrm{L} & 50 & 100 \\ \mu \mathrm{g} / \mathrm{L} & 50 & 100 \\ \mu \mathrm{g} / \mathrm{L} & 50 & 100 \\ \mu \mathrm{g} / \mathrm{L} & 2.5 & 5 \\ \mathrm{pCi} / \mathrm{L} & 1.5 \mathrm{E}+02 & 3 \mathrm{E}+02\end{array}$

\section{Source}

EPA Method 8270 EPA Method 8270 EPA Method 8240 EPA Method 8240 EPA Method 8270 EPA Method 8270 EPA Method 8240 EPA Method 8240 EPA Method 8080 Set by EPD/EMS EPA Method 8240 EP.A Method 6010 Proposed DWS (EPA, 1991) Proposed DWS (EPA, 1991) EPA Method 8270 APHA Method 417B EPA Method 350.1 EPA Method 8270 EPA Method 8270 Proposed DWS (EPA, 1990) Final DWS (EPA, 1977) 


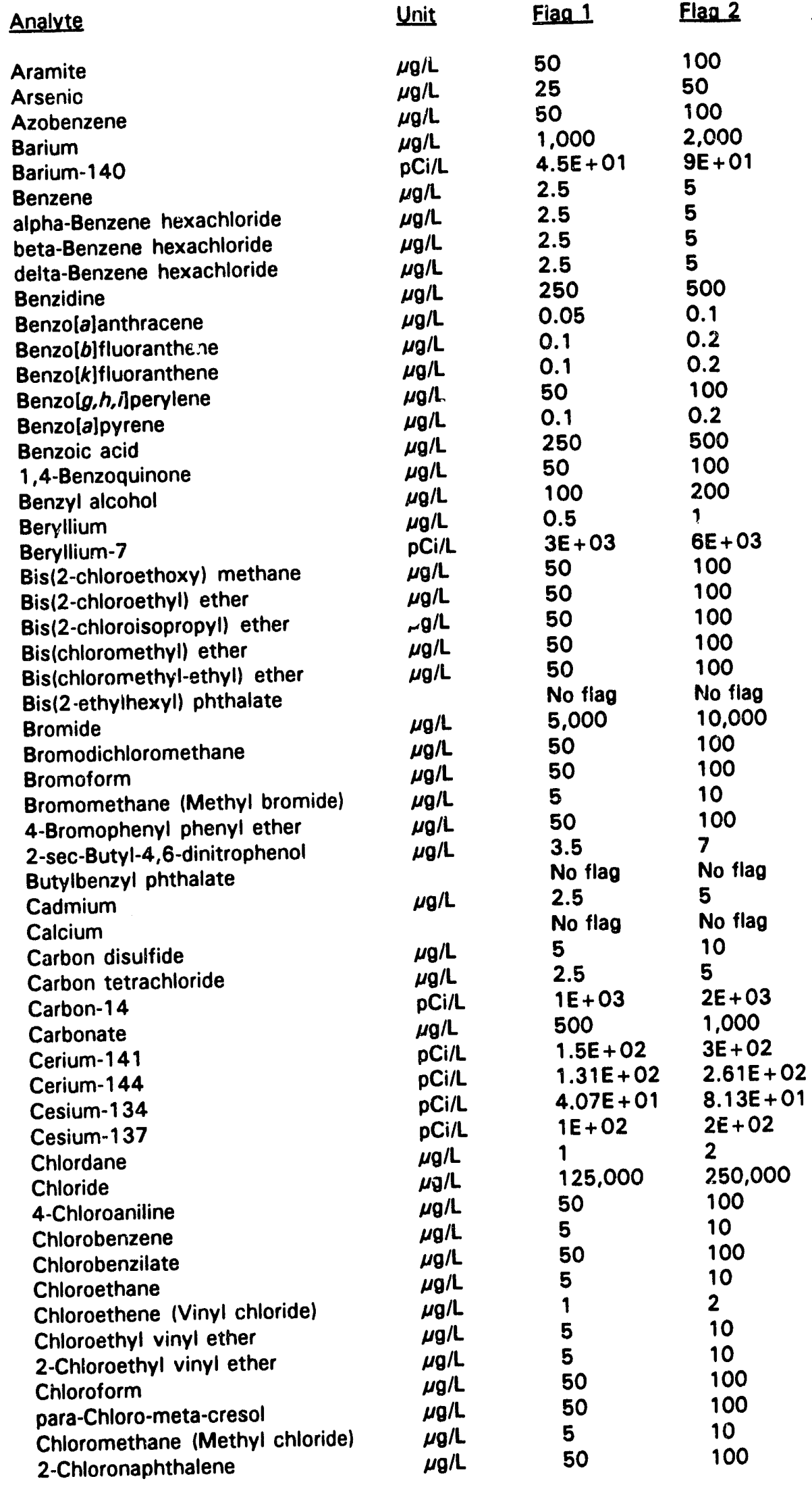

\section{Source}

EPA Method 8270

Final DWS (CFR, 1991a)

EPA Method 625

Final DWS (CFR, 1991a)

Final DWS (EPA, 1977)

Final DWS (CFR, 1991a)

EPA Method 8080

EPA Method 8080

EPA Mathod 8080

EPA Method 8270

Proposed DWS (EPA, 1990)

Proposed DWS (EPA, 1990)

Proposed DWS (EPA, 1990)

EPA Method 8270

Proposed DWS (EPA, 1990)

EPA Method 8270

EPA Method 8270

EPA Method 8270

Proposed DWS (EPA, 1990)

Final DWS (EPA, 1977)

EPA Method 8270

EPA Method 8270

EPA Method 8270

EPA. Method 8270

EFA Method 8270

Set by EPD/EMS

EPA Method 300.0

Final DWS (CFR, 1991a)

Final DWS (CFR, 1991a)

EPA Method 8240

EPA Method 8270

Proposed DWS (EPA, 1990)

Set by EPD/EMS

Final DWS (CFR, 1991a)

Set by EPD/EMS

EPA Method 8240

Final DWS (CFR, 1991a)

Final DWS (EPA, 1977)

EPA Method 310.1

Final DWS (EPA, 1977)

Proposed DWS (EPA, 1991)

Proposed DWS (EPA, 1991)

Final DWS (EPA, 1977)

Final DWS (CFR, 1991a)

Secondary DWS (CFR, 1991b)

EPA Method 8270

EPA Method 8240

EPA Method 8270

EPA Method 8240

Final DWS (CFR, 1991a)

EPA Method 8240

EPA Method 8240

Final DWS (CFR, 1991a)

EPA Method 8270

EPA Method 8240

EPA Method 8240 
Analyte

2-Chlorophenol

4-Chlorophenyl phenyl ether

Chloroprene

Chromium

Chromium-51

Chrysene

Cobalt

Cobalt-57

Cobalt-58

Cobalt-60

Color

Copper

Corrosivity

m-Cresol (3-Methylphenol)

o-Cresol (2-Methylphenol)

p-Cresol (4-Methylphenol)

Curium-242

Curium-243

Curium-244

Curium-246

Cyanide

$\hat{p}, p^{\prime}-D D D$

$p, p^{\prime}-D D E$

$p, p^{\prime}$-DDT

Di-n-butyl phthalate

Di-n-octyl phthalate

Diallate

Dibenzla,h]anthracene

Dibenzofuran

Dibromochloromethane

Dibromochloropropane

1,2-Dibromo-3-chlorcpropane

1,2-Dibromoethane

Dibromomethane

(Methylene bromide)

1,2-Dichlorobenzene

1,3-Dichlorobenzene

1,4-Dichlorobenzene

3,3'-Dichlorobenzidine

trans-1,4-Dichloro- -butene

Dichlorodifluorometirane

1,1-Dichloroethane

1,2-Dichloroethane

cis-1,2-Dichloroethene

1,1-Dichloroethylene

1,2-Dichloroethylene

trans-1,2-Dichloroethylene

Dichloromethane

(Methylene chloride)

2,4-Dichlorophenol

2,6-Dichlorophenol

2,4-Dichlorophenoxyacetic acid

1,2-Dichloropropane

cis-1,3-Dichloropropene

trans-1,3-Dichloropropene

Dieldrin

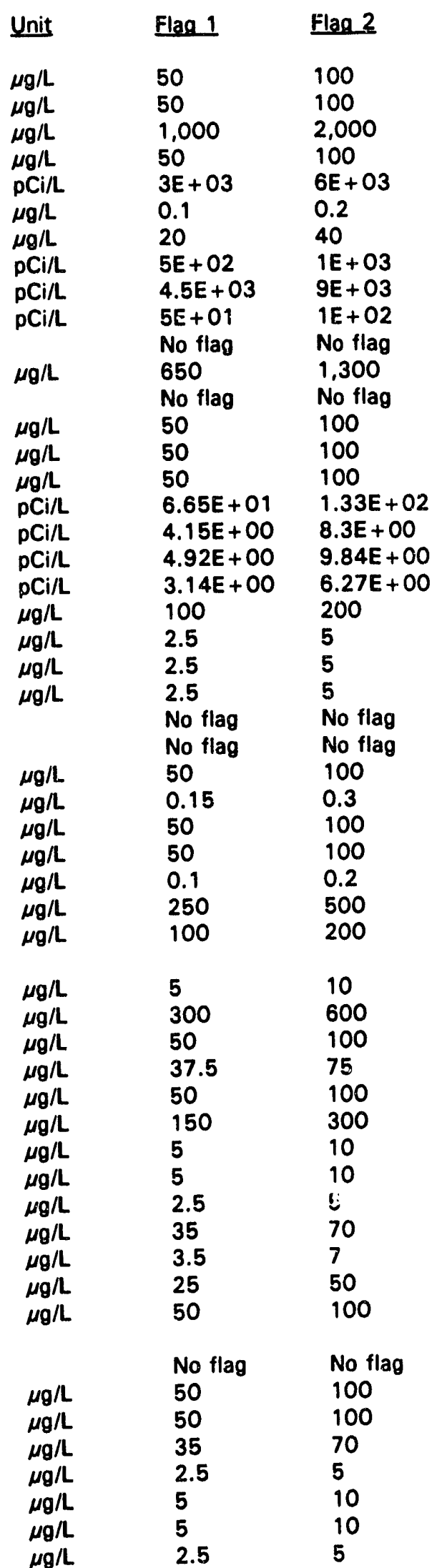

Source

EPA Method 8270

EPA Method 8270

EPA Method 8240

Final DWS (CFR, 1991a)

Final DWS (EPA, 1977)

Proposed DWS (EPA, 1990)

EPA Method 6010

Final DWS (EPA, 1977)

Final DWS (EPA, 1977)

Finai DWS (EPA, 1977)

Set by EPD/EMS

Final DWS (CFR, 1991a)

Set by EPD/EMS

EPA Method 8270

EPA Method 8270

EPA Method 8270

Proposed DWS (EPA, 1991)

Proposed DWS (EPA, 1991)

Proposed DWS (EPA, 1991)

Proposed DWS (EPA, 1991)

Proposed DW/S (EPA, 1990)

EPA Method 8080

EPA Method 8080

EPA Method 8080

Set by EPD/EMS

Set by EPD/EMS

EPA Method 8270

Proposed DWS (EPA, 1990i

EPA Method 8270

Final DWS (CFR, 1991a)

Final DWS (CFR, 1991a)

EPA Method 8240

EPA Method 8240

EPA Method 8240

Final DWS (CFR, 1991a)

EPA Method 8270

Final DWS (CFR, 1991a)

EPA Methiod 8270

EPA Method 8240

EPA Method 8240

EPA Method 8240

Final DWS (CFR, 1991a)

Final DWS (CFR, 1991a)

Final DWS (CFR, 1991a)

EPA Method 8240

Final DWS (CFR, 1991a)

Set by EPD/EMS

EPA Method 8270

EPA Method 8270

Final DWS (CFR, 1991a)

Final DWS ;CFR, 1991a)

EPA Method 3240

EPA Method 8240

EPA Method 8080 


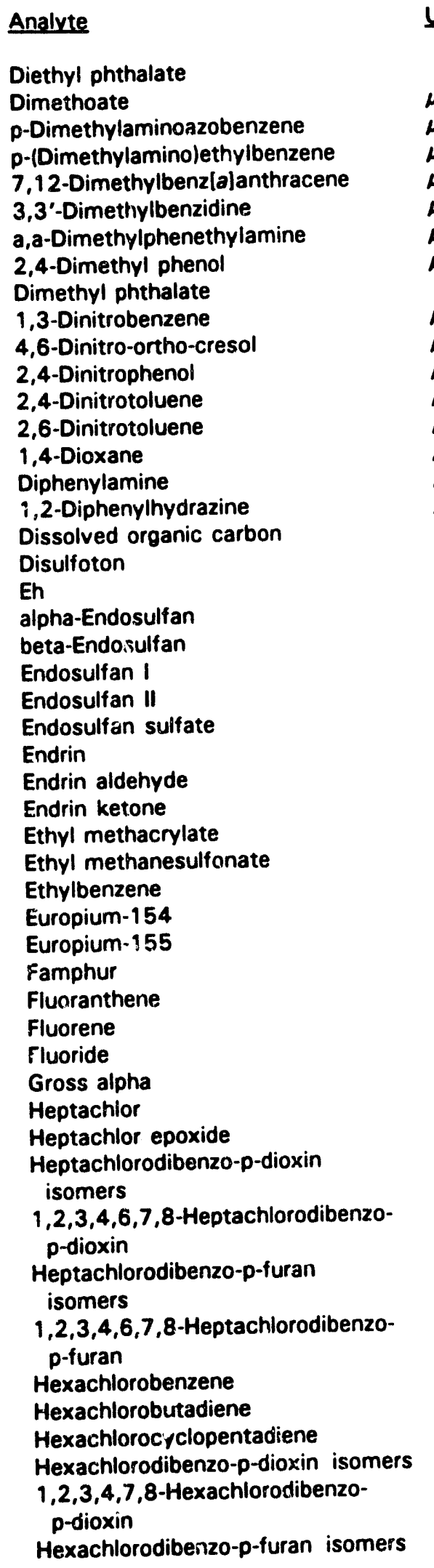

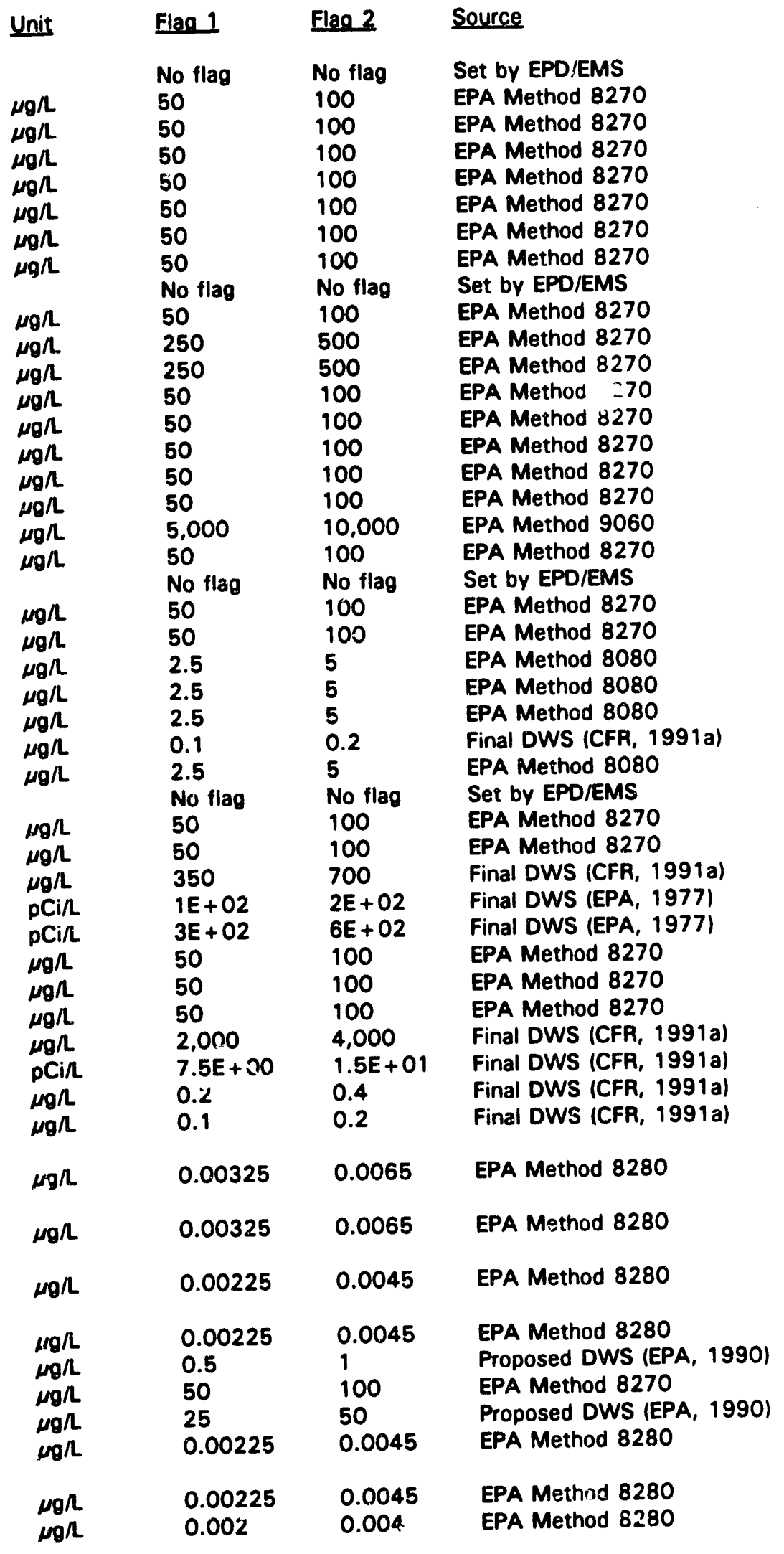


Analvte

$1,2,3,4,7,8-H$ exachlorodibenzop-furan

Hexachloroethane

Hexachlorophene

Hexachloropropene

2-Hexanone

Indenol1,2,3-c, olpyrene

lodine

lodine-129

lodine-131

lodomethane (Methyl iodide)

Iron

Iron-55

Iron-59

Isobutyl alcohol

Isodrin

Isophorone

Isosafrole

Kepone

Lanthanum-140

Lead

Lindane

Lithium

Magnesium

Manganese

Manganese-54

Mercury

Methacrylonitrile

Methapyrilene

Methoxychlor

3-Methylcholanthrene

2-Methyl-4,6-dinitrophenol

Methyl ethyl ketone

Methyl isobutyl ketone

Methyl methacrylate

Methyl methanesulfonate

2-Methylnaphthalene

Molybdenum

Naphthalene

1,4-Naphthoquinone

1-Naphthylamine

2-Naphthylamine

Neptunium-237

Nickel

Nickel-59

Nickel-63

Niobium-95

Nitrate as nitrogen

Nitrite as nitrogen

2-Nitroaniline

3-Nitroaniline

4-Nitroaniline

Nitrobenzene

Nitrogen by Kjeldahl method

2-Nitrophenol

4-Nitrophenol
Unit

\section{Flag 1}

Flag 2

Source

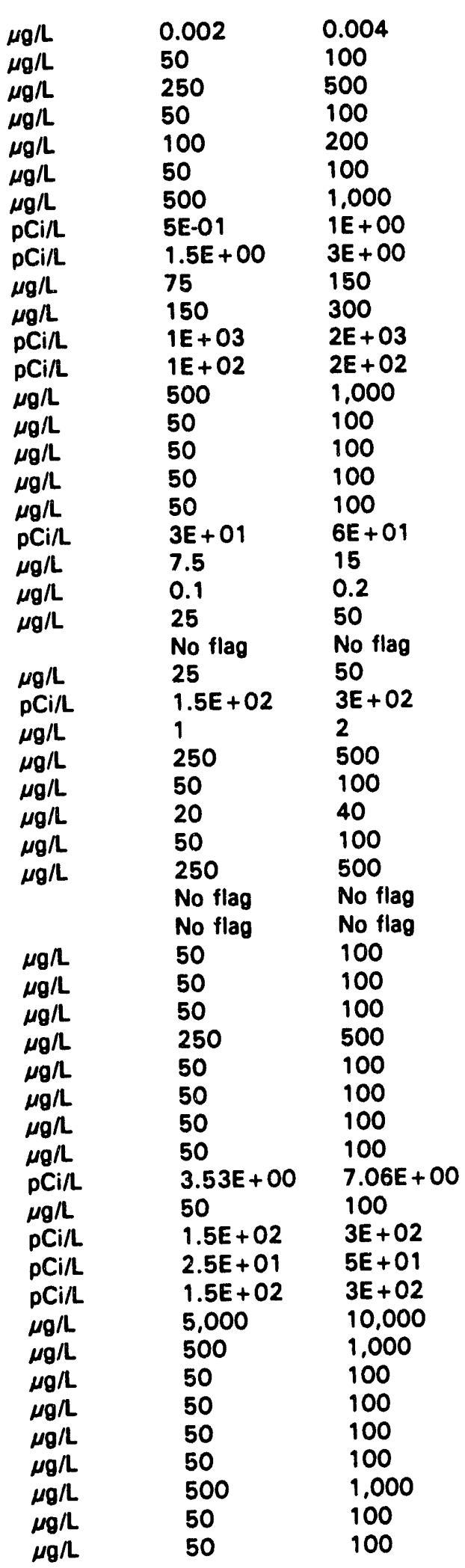

EPA Method 8280

EPA Method 8270

EPA Method 8270

EPA Method 8270

EPA Method 8240

EPA Method 8270

EPA Method 415

Final DWS (EPA, 1977)

Final DWS (EPA, 1977)

SPA Method 8240

Secondary DWS (CFR, 1991b)

Final DWS (EPA, 1977)

Final DWS (EPA, 1977)

EPA Method 8240

EPA Method 8270

EPA Method 8270

EPA Method 8270

EPA Method 8270

Final DWS (EPA, 1977)

Final DWS (CFR, 1991a)

Final DWS (CFR, 1991a)

EPA Method 6010

Set by EPD/EMS

Secondary DWS (CFR, 1991b)

Final DWS (EPA, 1977)

Final DWS (CFR, 1991a)

EPA Method 8240

EPA Method 8270

Final DWS (CFR, 1991a)

EPA Method 8270

EPA Method 8270

Set by EPD/EMS

Set by EPD/EMS

EPA Method 8270

EPA Method 8270

EPA Method 8270

EPA Method 6010

EPA Method 8270

EPA Method 8270

EPA Method 8270

EPA Method 8270

Proposed DWS (EPA, 1991)

Proposed DWS (EPA, 1990)

Final DWS (EPA, 1977)

Final DWS (EPA, 1977)

Final DWS (EPA, 1977)

Final DWS (CFR, 1991a)

Final DWS (CFR, 1991a)

EPA Method 8270

EPA Method 8270

EPA Method 8270

EPA Method 8270

EPA Method 351.2

EPA Method 8270

EPA Method 8270 


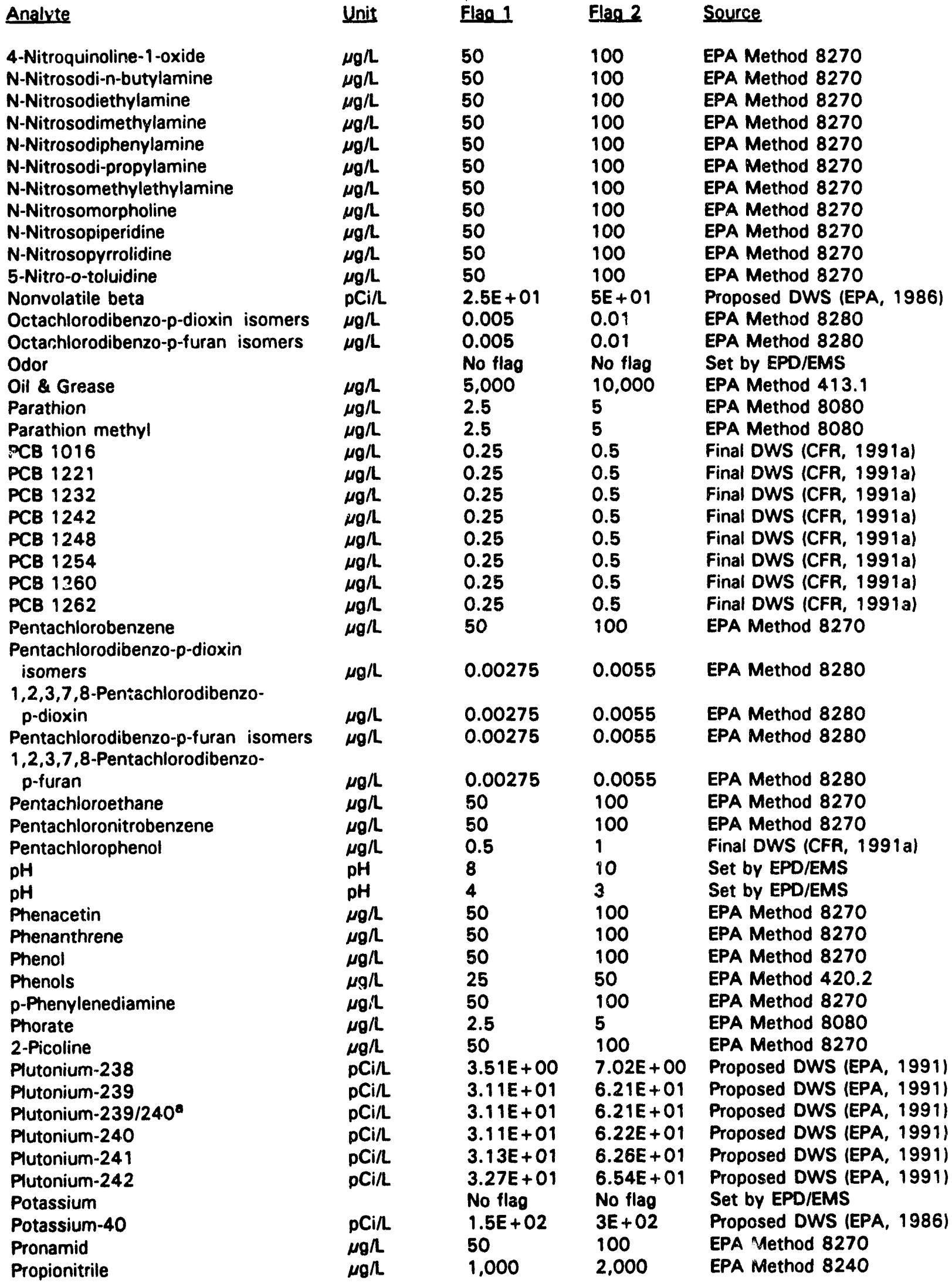




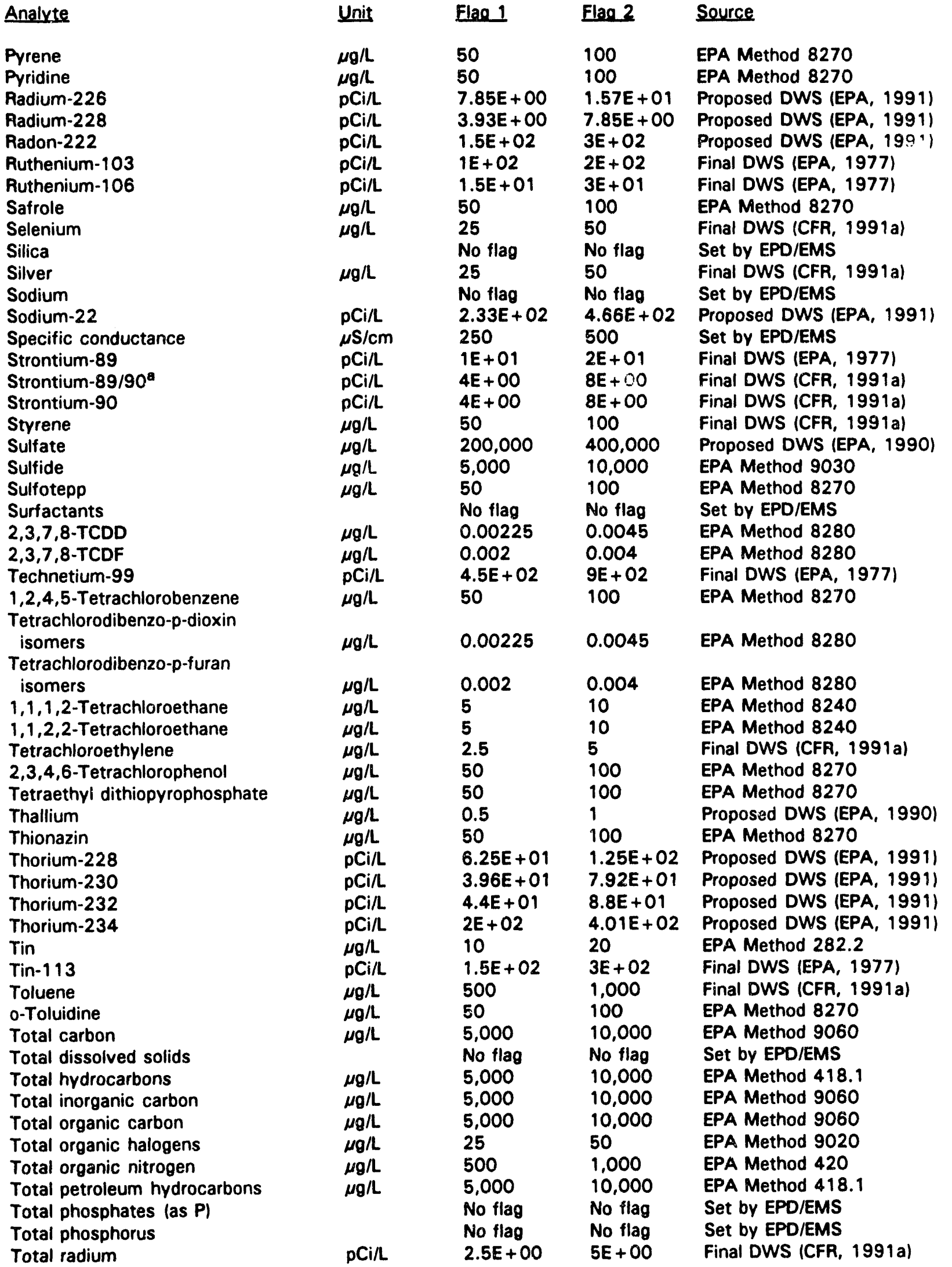




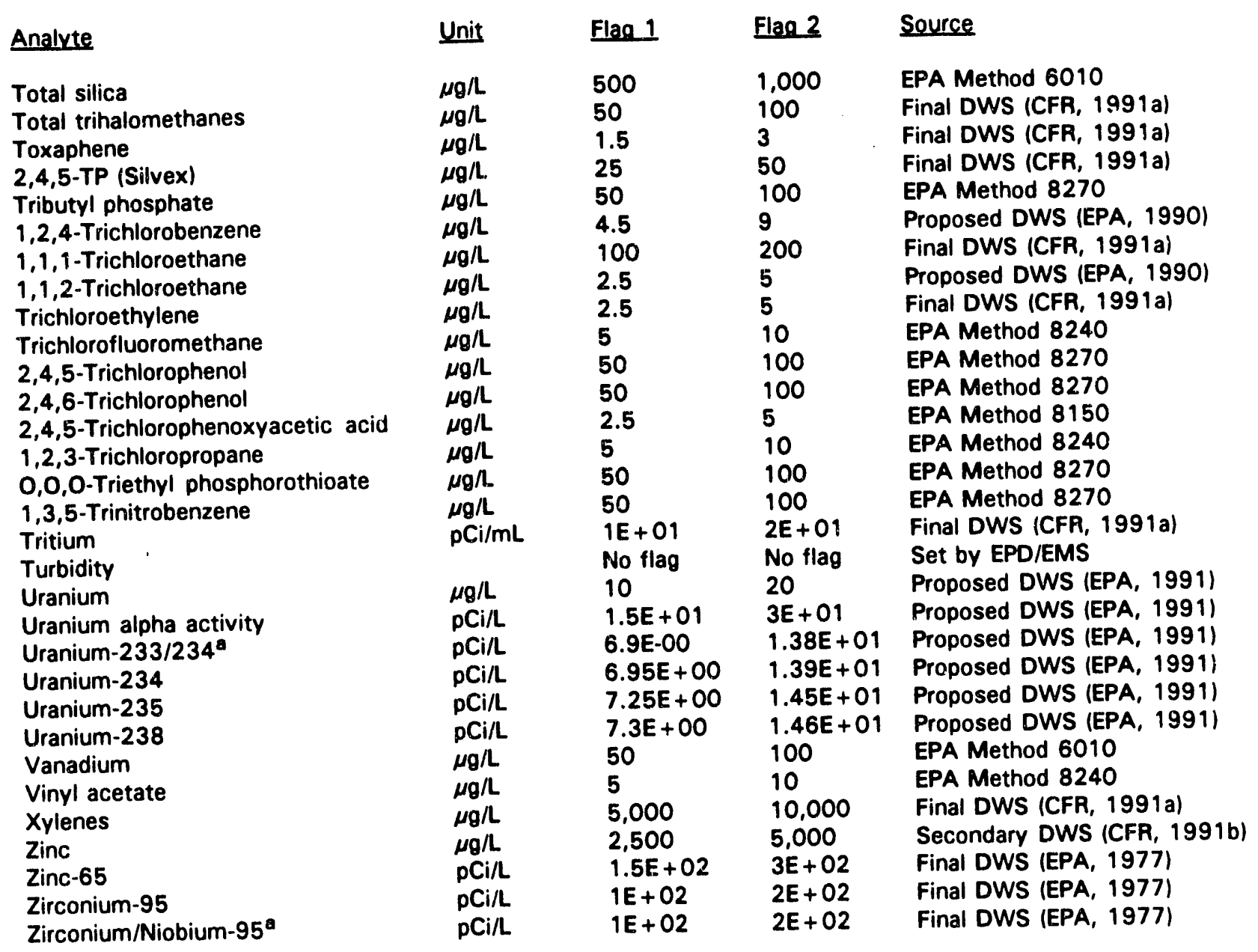

a For double radionuclide analyses where each separate radionuclide has its own standard, the more stringent standard is used.

\section{References}

APHA (American Public Health Association, American Water Works Association, and Water Pollution Control Federation), 1985. Standard Mothods for the Examination of Water and Wastewater, 16th edition. Washington, DC.

CFR (Code of Federal Regulations), 1991a. National Primary Drinking Water Regulations, 40 CFR, Part 141, pp. 578-715. Washington, DC.

CFR (Code of Federal Regulations), 1991b. National Secondary Drinking Water Regulations, 40 CFR, Part 143, pp. 758-762. Washington, DC.

EPA (U.S. Environmental Protection Agency), 1977. National Interim Primary Drinking Water Regulations, EPA-570/9-76-003. Washington, DC. 
EPA (U.S. Environmental Protection Agency), 1986. Water Pollution Control; National Primary Drinking Water Regulations, Radionuclides (Proposed). Federal Register, September 30, 1986, pp. 34836-34862. Washington, DC.

EPA (U.S. Environmental Protection Agency), 1990. National Primary and Secondary Drinking Water Regulations; Synthetic Organic Chemicals and Inorganic Chemicals (Proposed Rule). Federal Register, July 25, 1990. pp. 30369-30448. Washington, DC.

EPA (U.S. Environmental Protection Agency), 1991. National Primary Drinking Water Regulations; Radionuclides; Proposed Rule. Federal Register, July 18, 1991, pp. 33052-33127. Washington, DC. 


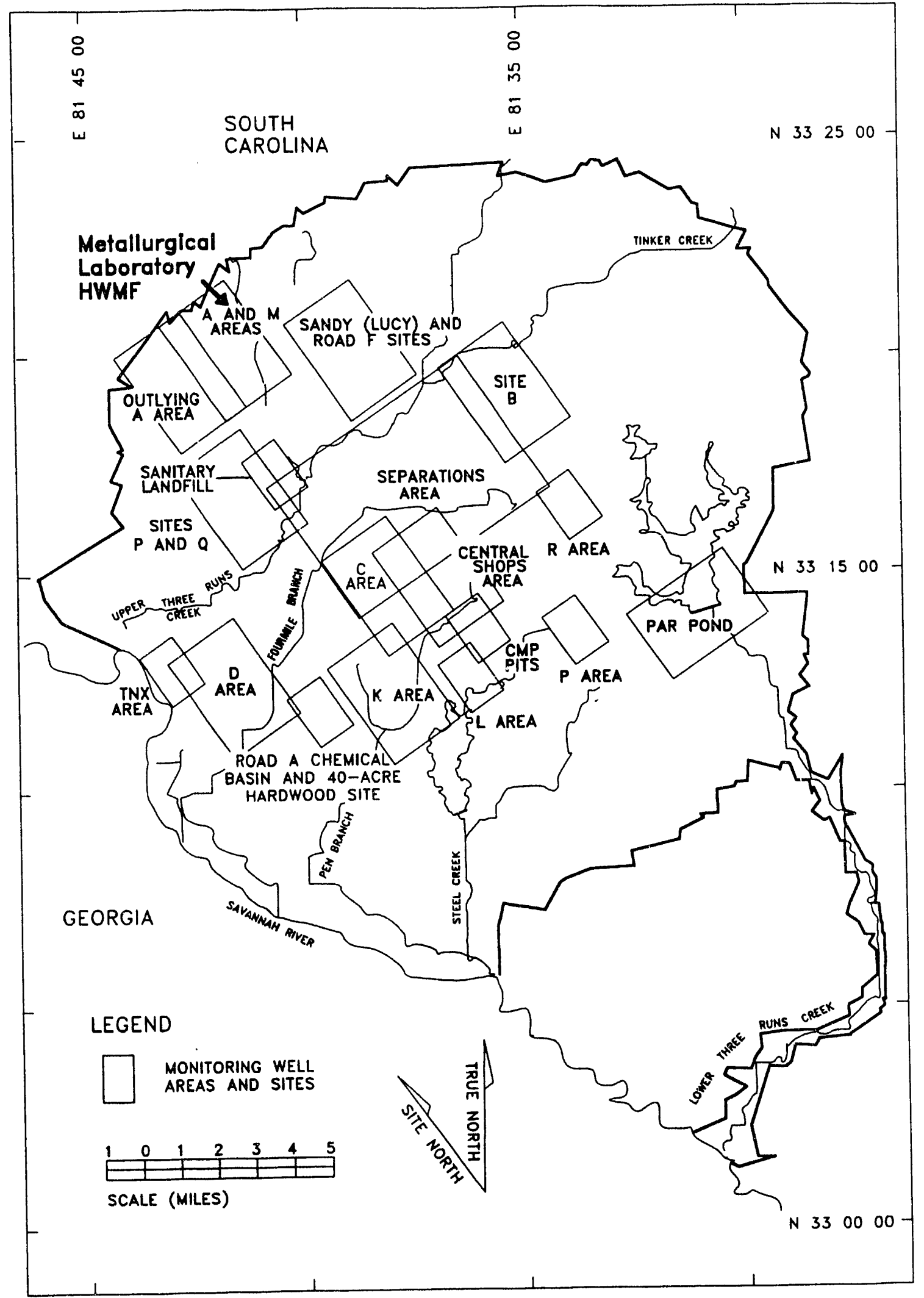

Figure 1. Location of the Metallurgical Laboratory HWMF at the Savannah River Site 


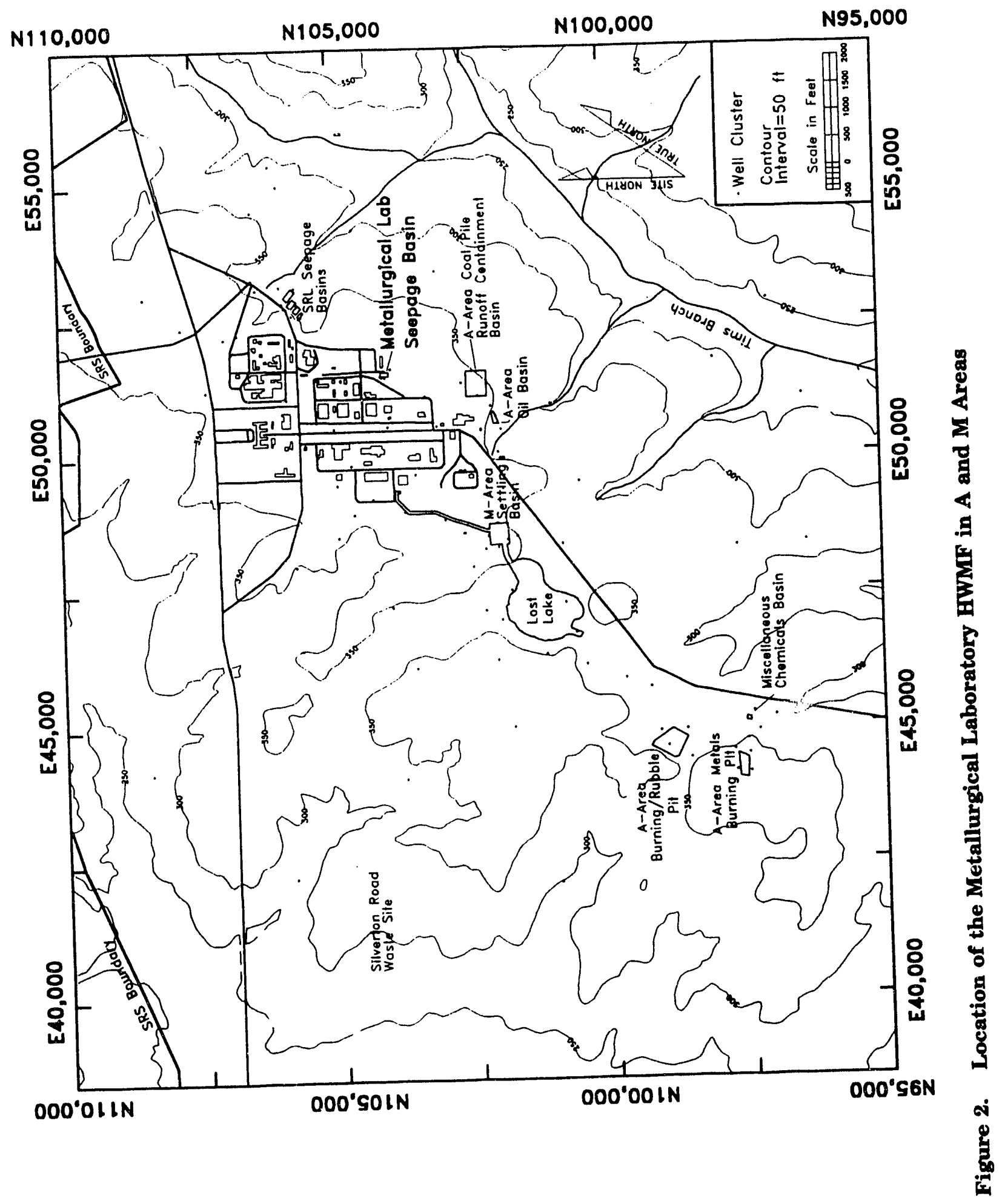




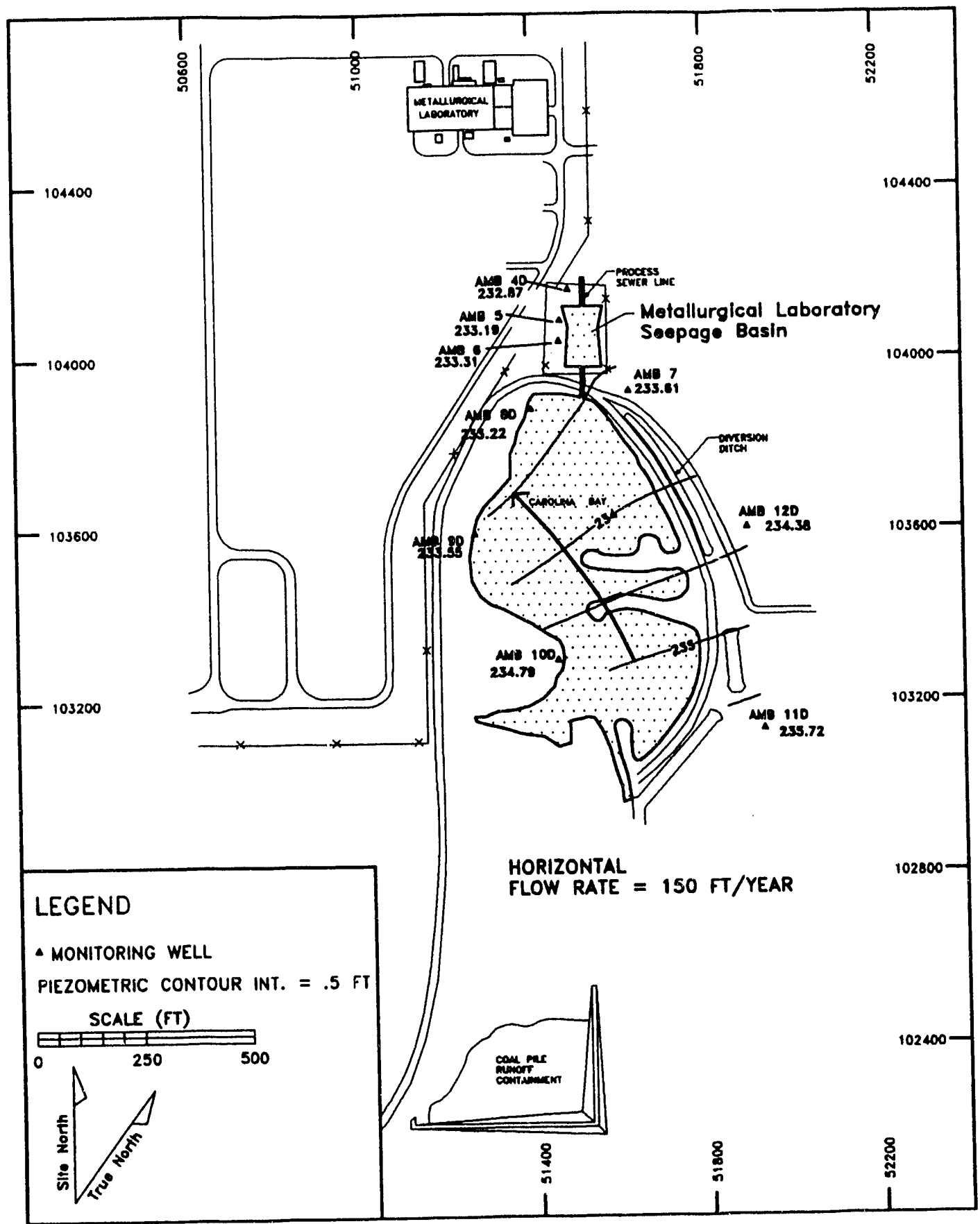

Figure 3. Water-Elevation Contour Map of the Water Table at the Metallurgical Laboratory HWMF and Locations of the Water-Table Wells 


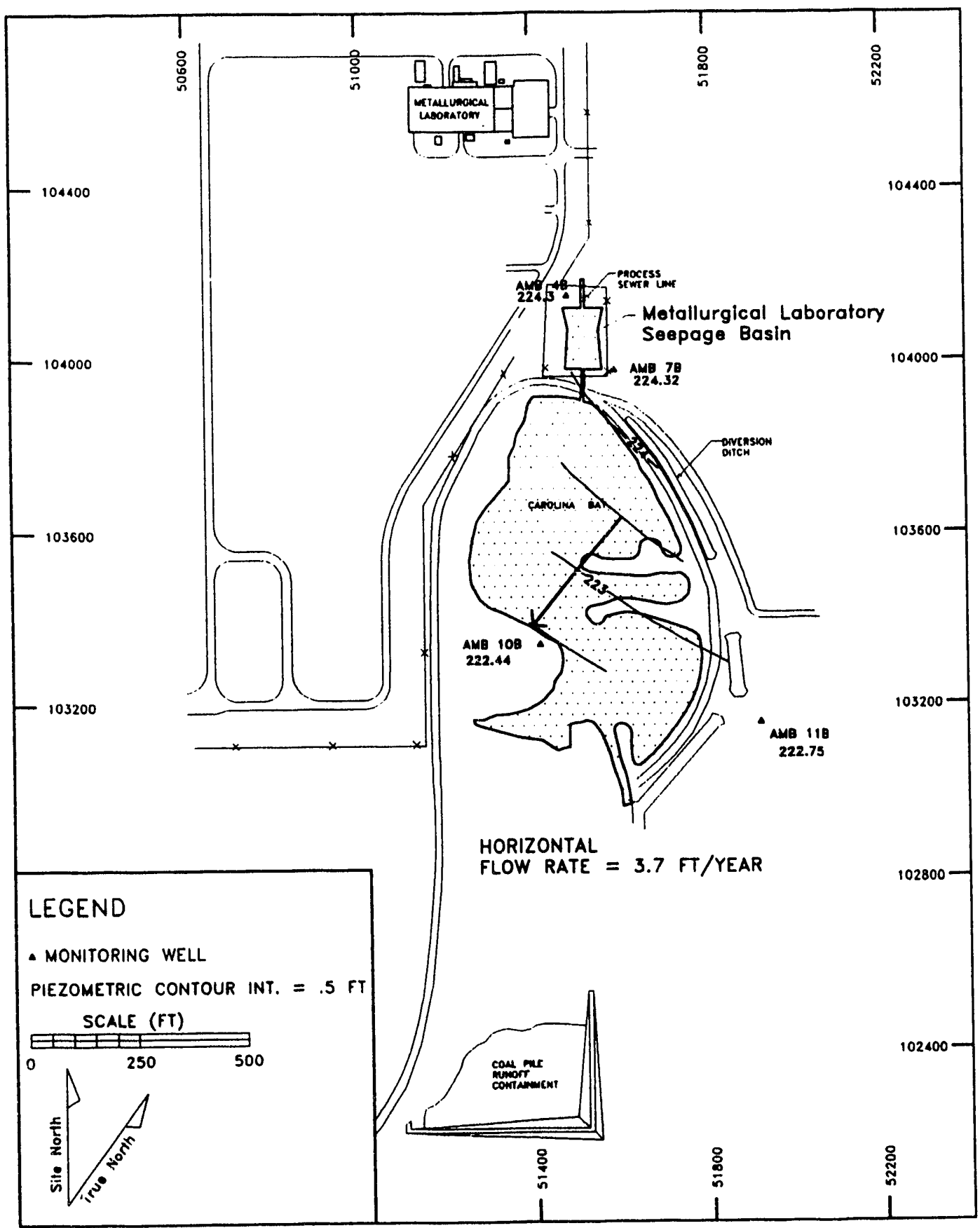

Figure 4. Potentiometric Surface Map of the Upper Section of the Congaree Aquifer at the Metallurgical Laboratory HWMF and Locations of Wells that Monitor the Upper Section of the Congaree 


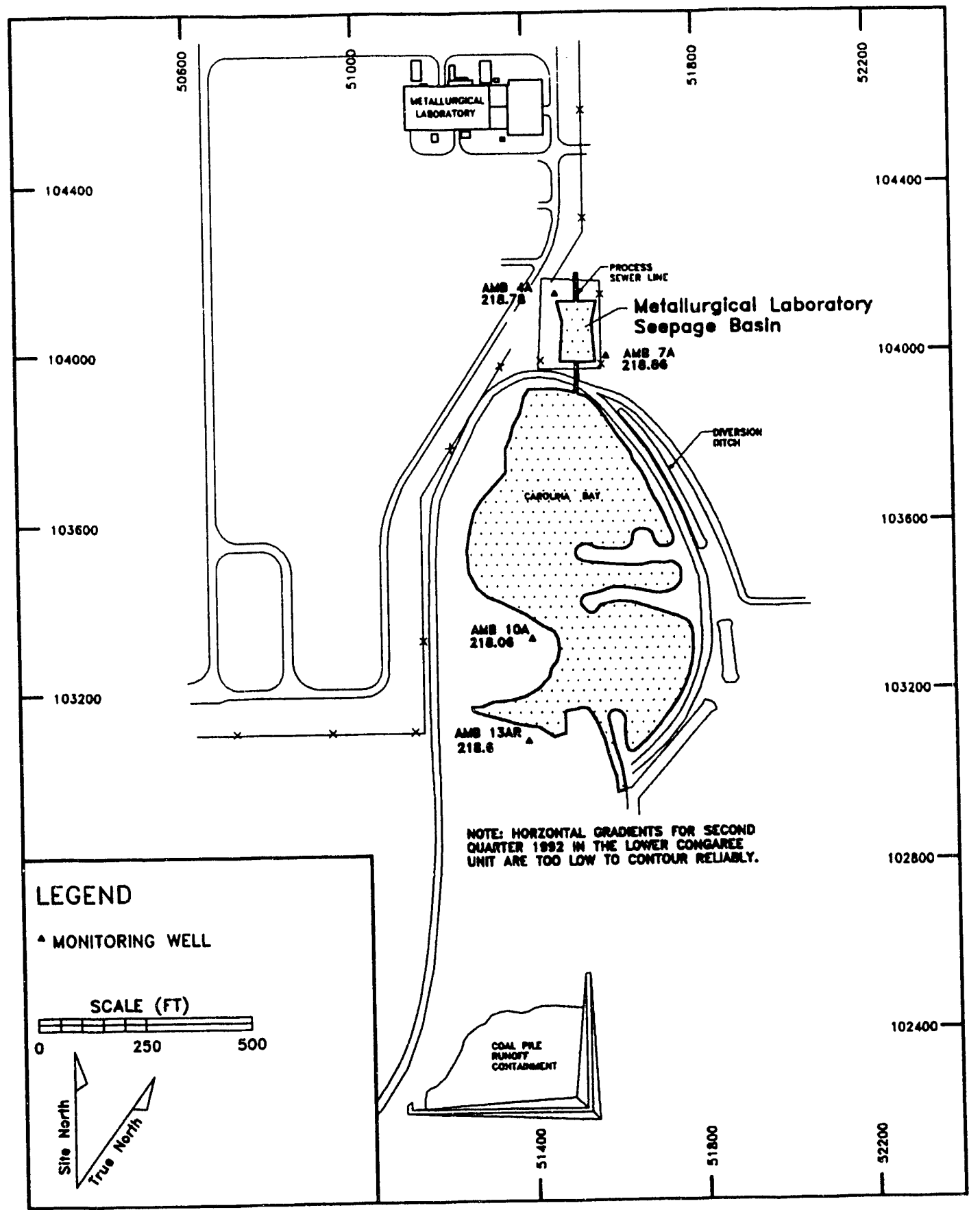

$\mathbf{C}$

$\mathbf{C}$

Figure 5. Potentiometric Surface Map of the Lower Section of the Congaree Aquifer at the Metallurgical Laboratory HWMF and Locations of Wells that Monitor the Lower Section of the Congaree 


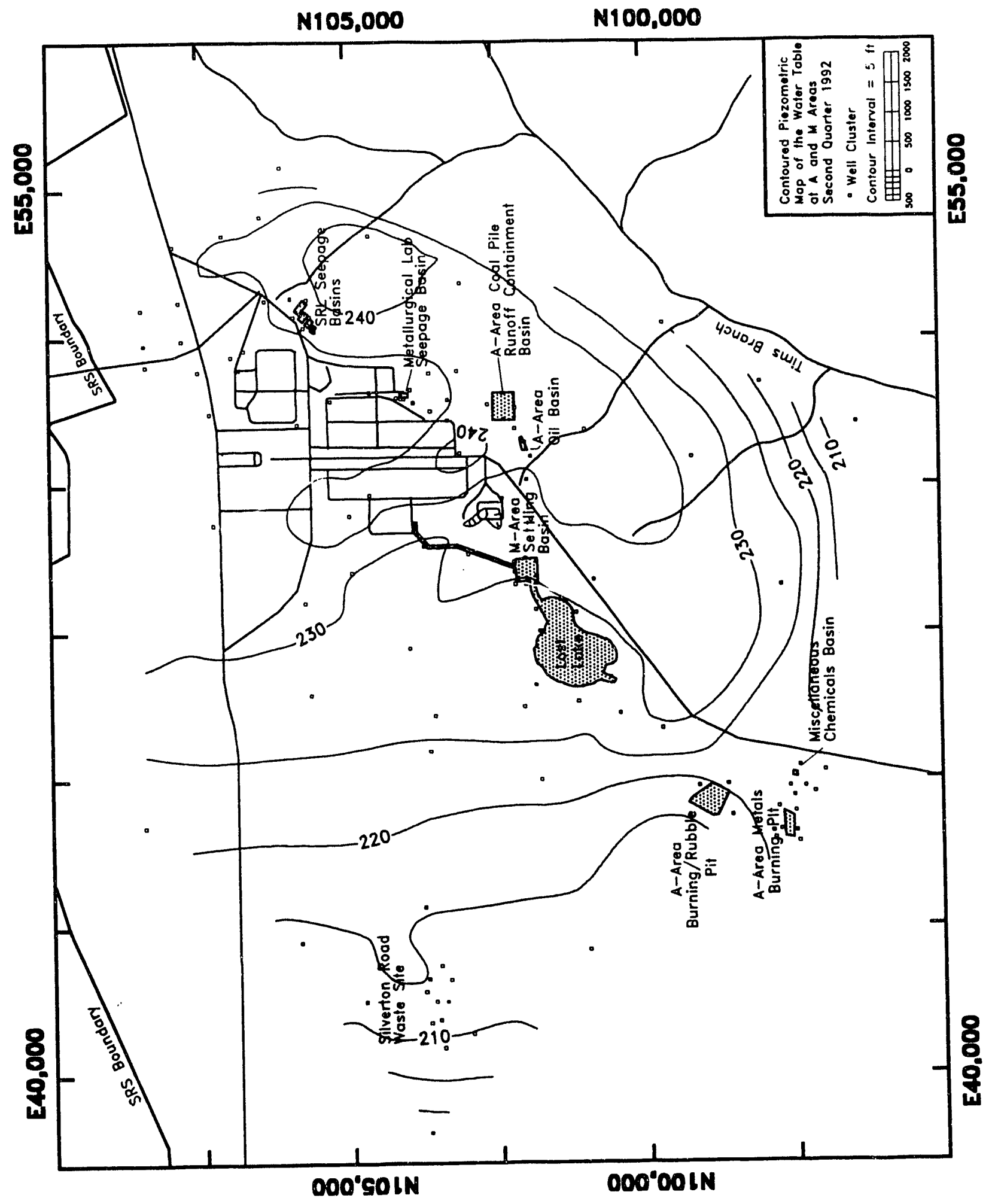

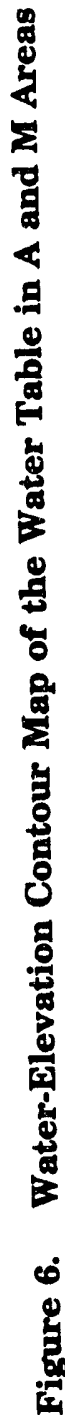


- Appendix D - Groundwater Monitoring Results Tables

-

- 


\section{Key to Reading the Tables}

The following abbreviations may appear in the tabular data:

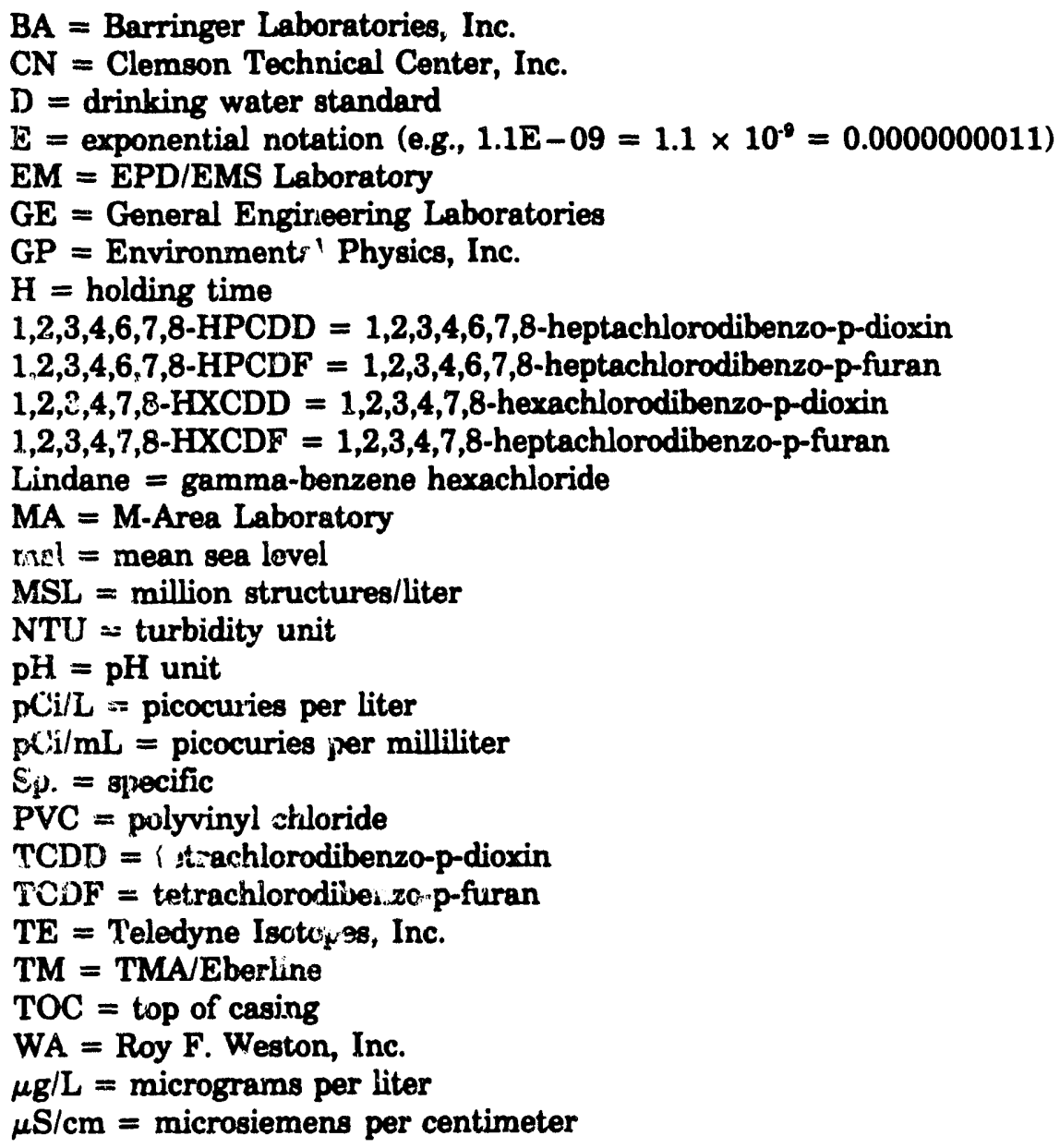

\section{Holding Times}

Standard analytical methods include a limit, called holding time, on the maximum elapsed time between sample collection and extraction or analysis by the laboratory. In the data tables, a large dot $(\bullet)$ in the $\mathrm{H}$ (holding time) column indicates that holding time was exceeded. Analyses performed beyond holding time may not yield valid results.

South Carolina Department of Health and Environmental Control (SCDHEC) allows only 15 minutes to elapse between sampling and analysis for $\mathrm{pH}$. Thus, laboratory $\mathrm{pH}$ analyses always exceed holding time.

Laboratory-initiated procedures for reducing the number of other analyses performed out of holding time include subcontracting analyses when difficulties with equipment, personnel, or work load would prevent timely analyses. Beginning fourth quarter 1991, SRS reduced the compensation to laboratories for analyses performed out of holding time. 
Table 1. Constituents Exceeding the Primary Drinking Water Standards

Aquifer Zone $\mathrm{IB}_{2}$ (Water Table)

\begin{tabular}{|c|c|c|c|c|c|}
\hline Well & & Constituent & Unit & 1092 & $\underline{2092}$ \\
\hline AMB & 4D & $\begin{array}{l}\text { Tetrachloroethylen? } \\
\text { Trichloroethylene }\end{array}$ & $\begin{array}{l}\mu g / L \\
\mu g / L\end{array}$ & $\begin{array}{l}-8 \\
67\end{array}$ & $\begin{array}{l}6.1 \\
86\end{array}$ \\
\hline AMB & 5 & $\begin{array}{l}\text { Tetrachloroethylene } \\
\text { Total alpha-emitting radiu:mb } \\
\text { Trichloroethylene }\end{array}$ & $\begin{array}{l}\mu g / L \\
\mathrm{pCi} / \mathrm{L} \\
\mu g / L\end{array}$ & $\begin{array}{l}8.4 \\
5.9 E+00 \\
140\end{array}$ & $\begin{array}{l}8.3 \\
6.3 E+00 \\
150\end{array}$ \\
\hline
\end{tabular}

Aquifer Unit ILA (upper section of Congaree)

$\begin{array}{lllll}\text { Well } & \text { Constituent } & \text { Unit } & 1092 & 2092 \\ \text { AMB 4B } & \text { Trichloroethylene } & \mu g / L & 7.7 & 9.9\end{array}$

Aquifer Unit lla (lower section of Congaree)

$\begin{array}{lllll}\text { Well } & \text { Constituent } & \text { Unit } & 1092 & \underline{2092} \\ \text { AMB 4A } & \begin{array}{l}\text { Tetrachloroethylene } \\ \text { Trichloroethylene } \\ \text { Trichloroethylene }\end{array} & \mu g / \mathrm{L} & 39 & 44 \\ & & \mu \mathrm{g} / \mathrm{L} & 462 & 812^{\circ} \\ & \mu g / \mathrm{L} & \mathrm{NA}^{\mathrm{d}} & 510^{\mathrm{C}} \\ \text { AMB 7A } & \begin{array}{l}\text { Tetrachloroethylene } \\ \text { Trichloroethylene }\end{array} & \mu \mathrm{g} / \mathrm{L} & 19 & 5.9 \\ & & \mu \mathrm{g} / \mathrm{L} & 450 & 104\end{array}$

a - = analyzed but not above the PDWS.

b The PDWS for total radium was applied to total alpha-emittinn radium.

c Duplicate samples of trichloroethylene.

d $N A=$ not analyzed. 
Table 2. Constituents Exceeding Half the Primary Drinking Water Standards or Other Flag 1 or Flag 2 Criteria

Aquifer Zone $\mathrm{IB}_{2}$ (Water Table)

\begin{tabular}{|c|c|c|c|c|c|}
\hline Well & & Constituent & Unit & $\underline{2092}$ & Flag \\
\hline AMB & 4D & $\begin{array}{l}\text { Manganese } \\
\text { Manganese } \\
\text { Total alpha-emitting radium } \\
\text { Total organic halogens }\end{array}$ & $\begin{array}{l}\mu g / L \\
\mu g / L \\
p C i / L \\
\mu g / L\end{array}$ & $\begin{array}{l}60^{a} \\
60^{a} \\
3.2 \\
57\end{array}$ & $\begin{array}{l}2 \\
2 \\
1 \\
2\end{array}$ \\
\hline AMB & 5 & Total organic halogens & $\mu g / L$ & 75 & 2 \\
\hline AMB & 6 & Trichloroethylene & $\mu g / L$ & 4.9 & 1 \\
\hline AMB & 10DD & Iron & $\mu g / L$ & 5,840 & 2 \\
\hline \multicolumn{6}{|c|}{ Aquifer Unit IIA (upper section of Congaree) } \\
\hline Well & & Constituent & Unit & 2092 & Flag \\
\hline AMB & 7B & Total organic halogens & $\mu g / L$ & 153 & 2 \\
\hline AMB & $11 \mathrm{~B}$ & $\begin{array}{l}\text { Antimony } \\
\text { Total alpha-emitting radium } \\
\text { Trichlorofluoromethane }\end{array}$ & $\begin{array}{l}\mu g / L \\
\mathrm{pCi} / \mathrm{L} \\
\mu g / L\end{array}$ & $\begin{array}{l}4.6 \\
3.4 \\
8.4\end{array}$ & $\begin{array}{l}1 \\
1 \\
1\end{array}$ \\
\hline
\end{tabular}

Aquifer Unit IIB (lower section of Congaree)

$\begin{array}{lllll}\text { Well } & \text { Constituent } & \text { Unit } & \underline{2092} & \underline{\text { Flag }} \\ \text { AMB 4A } & \text { Total organic halogens } & \mu g / L & 172 & 2 \\ \text { AMB 7A } & \text { Total organic halogens } & \mu g / L & 174 & 2 \\ \text { AMB 10A } & \begin{array}{l}\text { pH } \\ \text { Specific conductance }\end{array} & \begin{array}{l}\text { pH } \\ \mu S / c m\end{array} & 12 & 2\end{array}$

Note: Constituents exceeding half the PDWS appear italicized.

a Duplicate samples of manganese.

b The PDWS for total radium was applied to total alpha-emitting radium. 
Table 3. Groundwater Monitoring Results for Individual Wells

WELL AMB 4A

\begin{tabular}{|c|c|c|c|c|c|}
\hline sas coord. & Lat/Longitude & Screen Zone Elevation & Top of Casing & Casing & Formation \\
\hline $\begin{array}{l}\text { N104131.6 } \\
\text { E51469.8 }\end{array}$ & $\begin{array}{l}33.340041^{\circ} \mathrm{N} \\
81.733589{ }^{\circ} \mathrm{W}\end{array}$ & $126.3-121.3 \mathrm{ft} \mathrm{msl}$ & $380.5 \mathrm{ft} \mathrm{msl}$ & 4" PVC & Congaree (IIA) \\
\hline
\end{tabular}

MEASUREMENTS CONDUCTED IN THE FIELD

Sample date: $04 / 28 / 92$

Depth to water: $161.31 \mathrm{ft}(49.17 \mathrm{~m})$ below TOC

Water elevation: $219.19 \mathrm{ft}(66.81 \mathrm{~m}) \mathrm{ms} /$

Sp. conductance: $79 \mu \mathrm{S} / \mathrm{cm}$

Water evacuated before sampling: $257 \mathrm{gal}$

Time: $12: 20$

$\mathrm{pH}: 7.1$

Alkalinity: $26 \mathrm{mg} / \mathrm{L}$

Water temperature: $18.2^{\circ} \mathrm{C}$

\section{LABORATORY ANALYSES}

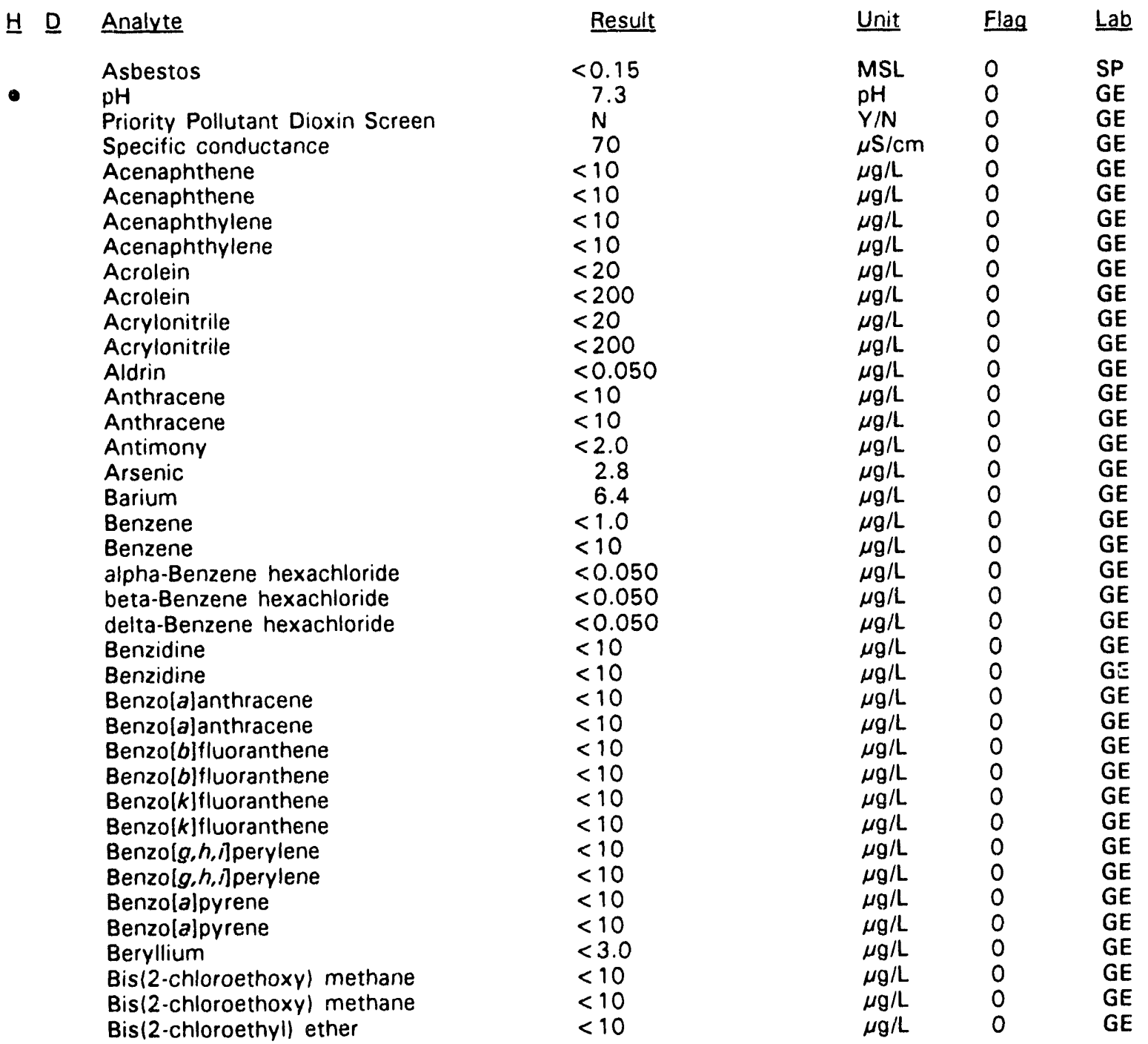

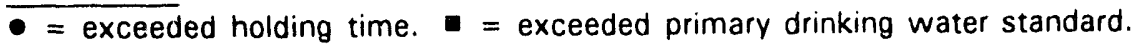


WELL AMB 4A collected on 04/28/92, laboratory analyses (cont.)

H D Analyte

Bis (2-chloroethyl) ether

Bis(2-chloroisopropyl) ether

Bis (2-chloroisopropyl) ether

Bis(2-ethylhexyl) phthalate

Bis(2-ethylhexyl) phthalate

Bromodichloromethane

Bromodichloromethane

Bromoform

Bromoform

Bromomethane (Methyl bromide)

Bromomethane (Methyl bromide)

4-Bromophenyl phenyl ether

4-Bromophenyl phenyl ether

Butylbenzyl phthalate

Butylbenzyl phthalate

Cadmium

Calcium

Carbon tetrachloride

Carbon tetrachloride

Chlordane

Chloride

Chlorobenzene

Chlorobenzene

para-Chloro-meta-cresol

para-Chluro-meta-cresol

Chloroethane

Chloroethane

Chloroethene (Vinyl chloride)

Chloroethene (Vinyl chloride)

2-Chloroethyl vinyl ether

2-Chloroethyl vinyl ether

Chloroform

Chloroform

Chloromethane (Methyl chloride)

Chloromethane (Methyl chloride)

2-Chloronaphthalene

2-Chloronaphthalene

2-Chlorophenol

2-Chlorophenol

4-Chlorophenyl phenyl ether

4-Chlorophenyl phenyl ether

Chromium

Chrysene

Chrysene

Copper

Cyanide

$p, p^{\prime}-D D D$

$p, p^{\prime}-D D E$

$p, p^{\prime}-D D T$

Dibenz[a, $h]$ anthracene

Dibenzla,h]anthracene

Dibromochloromethane

Dibromochloromethane

Di-n-butyl phthalate

Di-n-butyl phthalate

1,2-Dichlorobenzene
Result

$<10$

$<10$

$<10$

$<10$

$<10$

$<1.0$

$<10$

$<1.0$

$<10$

$<1.0$

$<10$

$<10$

$<10$

$<10$

$<10$

$<2.0$

7.640

$<1.0$

$<10$

$<0.50$

1.830

$<1.0$

$<10$

$<10$

$<10$

$<1.0$

$<10$

$<1.0$

$<10$

$<1.0$

$<10$

$<1.0$

$<10$

$<1.0$

$<10$

$<10$

$<10$

$<10$

$<10$

$<10$

$<10$

$<4.0$

$<10$

$<10$

$<4.0$

$<5.0$

$<0.10$

$<0.10$

$<0.10$

$<10$

$<10$

$<1.0$

$<10$

$<10$

$<10$

$<1.0$
Unit Flag Lab

$\mu g / L \quad 0 \quad$ GE

$\mu \mathrm{g} / \mathrm{L} \quad 0 \quad \mathrm{GE}$

$\mu \mathrm{g} / \mathrm{L} \quad \mathrm{O} \quad \mathrm{GE}$

$\mu \mathrm{g} / \mathrm{L} \quad 0 \quad \mathrm{GE}$

$\mu g / L \quad 0 \quad$ GE

$\mu g / L \quad 0 \quad G E$

$\mu g / L \quad 0 \quad$ GE

$\mu g / L \quad 0 \quad G E$

$\mu \mathrm{g} / \mathrm{L} \quad 0 \quad \mathrm{GE}$

$\mu g / L \quad 0 \quad$ GE

$\mu g / L \quad 0 \quad G E$

$\mu \mathrm{g} / \mathrm{L} \quad 0 \quad \mathrm{GE}$

$\mu \mathrm{g} / \mathrm{L} \quad \mathrm{O} \quad \mathrm{GE}$

$\mu \mathrm{g} / \mathrm{L} \quad 0 \quad \mathrm{GE}$

$\mu \mathrm{g} / \mathrm{L} \quad \mathrm{O} \quad \mathrm{GE}$

$\mu g / L \quad 0 \quad G E$

$\mu \mathrm{g} / \mathrm{L} \quad 0 \quad \mathrm{GE}$

$\mu \mathrm{g} / \mathrm{L} \quad 0 \quad \mathrm{GE}$

$\mu g / \mathrm{L} \quad 0 \quad \mathrm{GE}$

$\mu g / L \quad 0 \quad G E$

$\mu \mathrm{g} / \mathrm{L} \quad \mathrm{O} \quad \mathrm{GE}$

$\mu g / \mathrm{L} \quad 0 \quad \mathrm{GE}$

$\mu g / L \quad 0 \quad$ GE

$\mu \mathrm{g} / \mathrm{L} \quad 0 \quad \mathrm{GE}$

$\mu \mathrm{g} / \mathrm{L} \quad 0 \quad \mathrm{GE}$

$\mu g / L \quad 0 \quad G E$

$\mu \mathrm{g} / \mathrm{L} \quad 0 \quad \mathrm{GE}$

$\mu g / L \quad 0 \quad G E$

$\mu \mathrm{g} / \mathrm{L} \quad \mathrm{O} \quad \mathrm{GE}$

$\mu g / L \quad 0 \quad G E$

$\mu \mathrm{g} / \mathrm{L} \quad 0 \quad \mathrm{GE}$

$\mu g / L \quad 0 \quad G E$

$\mu \mathrm{g} / \mathrm{L} \quad \mathrm{O} \quad \mathrm{GE}$

$\mu g / L \quad 0 \quad G E$

$\mu g / L \quad 0 \quad G E$

$\mu \mathrm{g} / \mathrm{L} \quad 0 \quad \mathrm{GE}$

$\mu \mathrm{g} / \mathrm{L} \quad 0 \quad \mathrm{GE}$

$\mu g / L \quad 0 \quad$ GE

$\mu \mathrm{g} / \mathrm{L} \quad 0 \quad \mathrm{GE}$

$\mu \mathrm{g} / \mathrm{L} \quad \mathrm{O} \quad \mathrm{GE}$

$\mu \mathrm{g} / \mathrm{L} \quad \mathrm{O} \quad \mathrm{GE}$

$\mu \mathrm{g} / \mathrm{L} \quad \mathrm{O} \quad \mathrm{GE}$

$\mu \mathrm{g} / \mathrm{L} \quad \mathrm{O} \quad \mathrm{GE}$

$\mu \mathrm{g} / \mathrm{L} \quad \mathrm{O} \quad \mathrm{GE}$

$\mu g / L \quad 0 \quad G E$

$\mu g / L \quad 0 \quad G E$

$\mu g / L \quad 0 \quad G E$

$\mu g / L \quad 0 \quad G E$

$\mu \mathrm{g} / \mathrm{L} \quad 0 \quad \mathrm{GE}$

$\mu g / L \quad 0 \quad G E$

$\mu g / L \quad 0 \quad G E$

$\mu \mathrm{g} / \mathrm{L} \quad 0 \quad \mathrm{GE}$

$\mu g / L \quad 0 \quad G E$

$\mu \mathrm{g} / \mathrm{L} \quad 0$

$\mu \mathrm{g} / \mathrm{L} \quad 0 \quad \mathrm{GE}$

$\mu \mathrm{g} / \mathrm{L}$

- =xceeded holding time. = exceeded primary drinking water standard. 
WELL AMB $4 A$ collected on $04 / 28 / 92$, laboratory analyses (cont.)

H D Analyte

1,2-Dichlorobenzene

1,3-Dichlorobenzene

1,3-Dichlorobenzene

1,4-Dichlorobenzene

1,4-Dichlorobenzene

3, '3'-Dichlorobenzidine

3,3'-Dichlorobenzidine

Dichlorodifluoromethane

Dichlorodifluoromethane

1,1-Dichloroethane

1,1-Dichloroethane

1,2-Dichloroethane

1,2-Dichloroethane

1,1-Dichloroethylene

1,1-Dichloroethylene

trans-1,2-Dichloroethylene

trans-1,2-Dichloroethylene

Dichloromethane (Methylene chloride)

Dichloromethane (Methylene chloride)

2,4-Dichlorophenol

2,4-Dichlorophenol

1,2-Dichloropropane

1,2-Dichloropropane

cis-1,3-Dichloropropene

cis-1,3-Dichloropropene

trans-1,3-Dichloropropene

trans-1,3-Dichloropropene

Dieldrin

Diethyl phthalate

Diethyl phthalate

2,4-Dimethyl phenol

2,4-Dimethyl phenol

Dimethyl phthalate

Dimethyl phthalate

2,4-Dinitrophenol

2,4-Dinitrophenol

2,4-Dinitrotoluene

2,4-Dinitrotoluene

2,6-Dinitrotoluene

2,6-Dinitrotoluene

Di-n-octyl phthalate

Di-n-octyl phthalate

1,2-Diphenylhydrazine

1,2-Diphenylhydrazine

Endosulfan I

Endosulfan II

Endosulfan sulfate

Endrin

Endrin aldehyde

Ethylbenzene

Ethylbenzene

Fluoranthene

Fluoranthene

Fluorene

Fluorene

Fluoride
Result

$<10$

$<1.0$

$<10$

$<1.0$

$<10$

$<10$

$<10$

$<1.0$

$<10$

$<1.0$

$<10$

$<1.0$

$<10$

$<1.0$

$<10$

$<1.0$

$<10$

3.2

$<10$

$<10$

$<10$

$<1.0$

$<10$

$<1.0$

$<10$

$<1.0$

$<10$

$<0.50$

$<10$

$<10$

$<10$

$<10$

$<10$

$<10$

$<45$

$<45$

$<10$

$<10$

$<10$

$<10$

$<10$

$<10$

$<10$

$<10$

$<0.10$

$<0.10$

$<0.10$

$<0.0060$

$<0.10$

$<1.0$

$<10$

$<10$

$<10$

$<10$

$<10$

$<100$
Unit Flag Lab

$\mu \mathrm{g} / \mathrm{L}$

$\mu \mathrm{g} / \mathrm{L}$

$\mu \mathrm{g} / \mathrm{L}$

$\mu g / L$

$\mu \mathrm{g} / \mathrm{L}$

$\mu g / L$

$\mu g / L$

$\mu g / L$

$\mu g / L$

$\mu \mathrm{g} / \mathrm{L}$

$\mu g / L$

$\mu \mathrm{g} / \mathrm{L}$

$\mu \mathrm{g} / \mathrm{L}$

$\mu g / L$

$\mu g / L$

$\mu g / L$

$\mu g / L$

$\mu \mathrm{g} / \mathrm{L}$

$\mu g / L$

$\mu g / L$

$\mu g / L$

$\mu \mathrm{g} / \mathrm{L}$

$\mu g / L$

$\mu \mathrm{g} / \mathrm{L}$

$\mu g / L$

$\mu g / L$

$\mu g / L$

$\mu g / L$

$\mu \mathrm{g} / \mathrm{L}$

$\mu \mathrm{g} / \mathrm{L}$

$\mu g / L$

$\mu \mathrm{g} / \mathrm{L}$

$\mu \mathrm{g} / \mathrm{L}$

$\mu g / L$

$\mu \mathrm{g} / \mathrm{L}$

$\mu \mathrm{g} / \mathrm{L}$

$\mu \mathrm{g} / \mathrm{L}$

$\mu \mathrm{g} / \mathrm{L}$

$\mu \mathrm{g} / \mathrm{L}$

$\mu \mathrm{g} / \mathrm{L}$

$\mu \mathrm{g} / \mathrm{L}$

$\mu \mathrm{g} / \mathrm{L}$

$\mu g / L$

$\mu \mathrm{g} / \mathrm{L}$

$\mu g / L$

$\mu \mathrm{g} / \mathrm{L}$

$\mu g / L$

$\mu \mathrm{g} / \mathrm{L}$

$\mu g / L$

$\mu \mathrm{g} / \mathrm{L}$

$\mu \mathrm{g} / \mathrm{L}$

$\mu g / L$

$\mu \mathrm{g} / \mathrm{L}$

$\mu g / L$

$\mu \mathrm{g} / \mathrm{L}$

$\mu \mathrm{g} / \mathrm{L}$
GE

GE

GE

GE

$G E$

GE

GE

GE

GE

$G E$

GE

GE

GE

GE

GE

$\mathrm{GE}$

GE

GE

GE

GE

GE

GE

GE

GE

GE

GE

GE

GE

GE

GE

GE

GE

GE

GE

GE

GE

GE

GE

GE

GE

GE

GE

GE

GE

GE

GE

GE

GE

GE

GE

GE

GE

GE

GE

GE

- exceeded holding time. $\quad$ = exceeded primary drinking water standard. 
WELL AMB 4A collected on 04/28/92, laboratory analyses (cont.)

H D Analvte

Heptachlor
Heptachlor epoxide
Hexachlorobenzene
Hexachlorobenzene
Hexachlorobutadiene
Hexachlorobutadiene
Hexachlorocyclopentadiene
Hexachlorocyclopentadiene
Hexachloroethane
Hexachloroethane
Indenol1,2,3-C, dpyrene
Indenol1,2,3-C, dpyrene
Iron
Isophorone
Isophorone
Lead
Lindane
Magnesium
Manganese
Mercury
2-Methyl-4,6-dinitrophenol
2-Methyl-4,6-dinitrophenol
Naphthalene
Naphthalene
Nickel
Nitrate as nitrogen
Nitrate as nitrogen
Nitrobenzene
Nitrobenzene
2-Nitrophenol
2-Nitrophenol
4-Nitrophenol
4-Nitrophenol
N-Nitrosodimethylamine
N-Nitrosodimethylamine
N-Nitrosodiphenylamine
N-Nitrosodiphenylamine
N-Nitrosodipropylamine
N-Nitrosodipropylamine
PCB 1016
PCB 1221
PCB 1232
PCB 1242
PCB 1248
PCB 1254
PCB 1260
Pentachlorophenol
Pentachlorophenol
Phenanthrene
Phenanthrene
Phenol
Phenol
Phenols
Phenols
Potassium
Pyrene

Result

$<0.050$

$<0.050$

$<10$

$<10$

$<10$

$<10$

$<10$

$<10$

$<10$

$<10$

$<10$

$<10$

9.7

$<10$

$<10$

$<3.0$

$<0.0050$

307

7.4

$<0.20$

$<10$

$<10$

$<10$

$<10$

$<4.0$

970

950

$<10$

$<10$

$<10$

$<10$

$<10$

$<10$

$<10$

$<10$

$<10$

$<10$

$<10$

$<10$

$<0.50$

$<0.50$

$<0.50$

$<0.50$

$<0.50$

$<0.50$

$<0.50$

$<10$

$<10$

$<10$

$<10$

$<10$

$<10$

$<5.0$

$<5.0$

1,120 
WELL AMB 4A collected on 04/28/92, laboratory analyses (cont.)

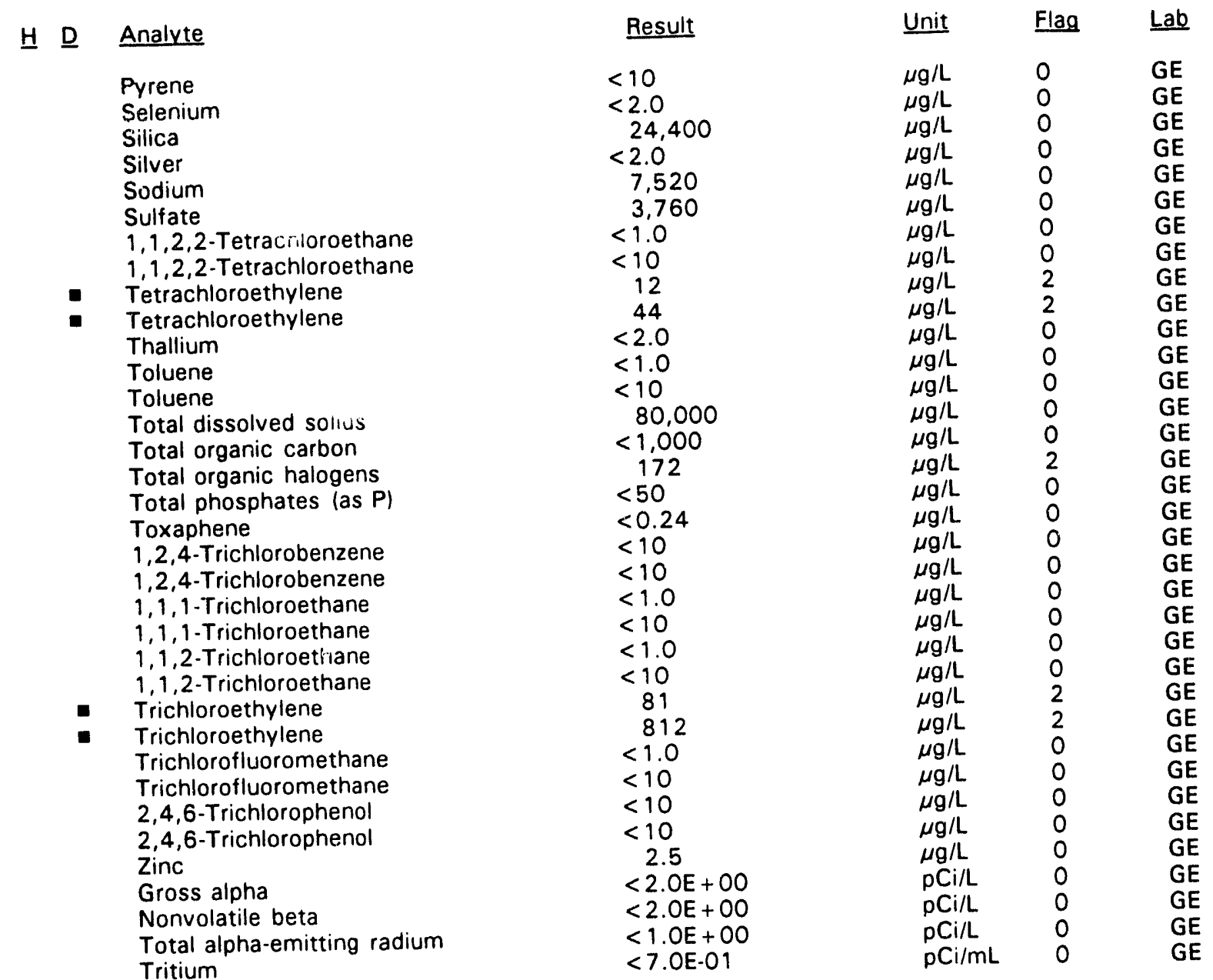

$\overline{\bullet=\text { exceeded holding time. }} \mathbf{-}=$ exceeded primary drinking water standard. 
WELL AMB 4B

\begin{tabular}{|c|c|c|c|c|c|}
\hline SRS Coord. & Lat/Longitude & Screen Zone Elevation & Top of Casing & Casing & Formation \\
\hline $\begin{array}{l}\text { N104145.6 } \\
\text { E5 } 1482.7\end{array}$ & $\begin{array}{l}33.340093^{\circ} \mathrm{N} \\
81.733582^{\circ} \mathrm{W}\end{array}$ & $157.3-152.3 \mathrm{ft} \mathrm{msl}$ & $380.4 \mathrm{ft} \mathrm{msl}$ & 4" PVC & ree (IIA) \\
\hline
\end{tabular}

MEASUREMENTS CONDUCTED IN THE FIELD

Sample date: 04/28/92

Depth to water: $155.90 \mathrm{ft}(47.52 \mathrm{~m})$ below TOC

Water elevation: $224.50 \mathrm{ft}(68.43 \mathrm{~m}) \mathrm{msl}$

Sp. conductance: $30 \mu \mathrm{S} / \mathrm{cm}$

Water evacuated before sampling: $189 \mathrm{gal}$

\section{LABORATORY ANALYSES}

H D Analyte

Asbestos

$\mathrm{pH}$

Priority Pollutant Dioxin Screen

Specific conductance

Acenaphthene

Acenaphthylene

Acrolein

Acrylonitrile

Aldrin

Aldrin

Anthracene

Antimony

Arsenic

Barium

Benzene

alpha-Benzene hexachloride

alpha-Benzene hexachloride

beta-Benzene hexachloride

beta-Benzene hexachloride

delta-Benzene hexachloride

delta-Benzene hexachloride

Benzidine

Benzolalanthracene

Benzolb]fluoranthene

Benzo[k]fluoranthene

Benzolg, $h, i]$ perylene

Benzola]pyrene

Beryllium

Bis(2-chloroethoxy) methane

Bis(2-chloroethyl) ether

Bis(2-chloroisopropyl) ether

Bis(2-ethylhexyl) phthalate

Bromodichloromethane

Bromoform

Bromomethane (Methyl bromide)

4-Bromophenyl phenyl ether

Butylbenzyl phthalate

Cadmium

Calcium
Time: $11: 35$

pH: 4.8

Alkalinity: $0 \mathrm{mg} / \mathrm{L}$

Water temperature: $17.5^{\circ} \mathrm{C}$

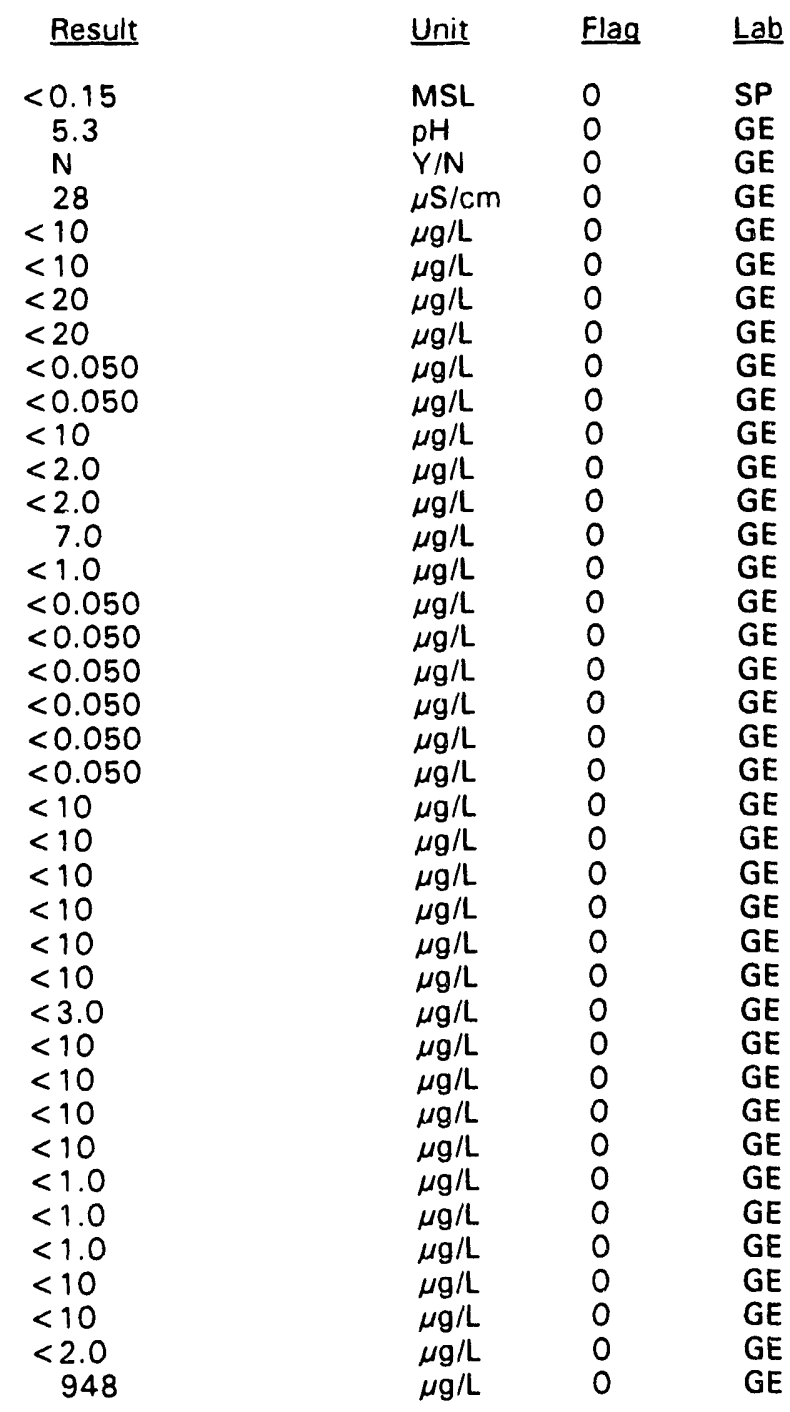

= exceeded holding time.

= exceeded primary drinking water standard.

Metallurgical Laboratory HWMF

D-10

Second Quarter 1992 
WELL AMB 4B collected on 04/28/92, laboratory analyses (cont.)

H D Analyte

Carbon tetrachloride
Chlordane

Chlordane

Chloride

Chloride

Chlorobenzene

para-Chloro-meta-cresol

Chloroethane

Chloroethene (Vinyl chlor:;e)

2-Chloroethyl vinyl ether

Chloroform

Chloromethane (Methyl chloride)

2-Chloronaphthalene

2-Chlorophenol

4-Chlorophenyl phenyl ether

Chromium

Chrysene

Copper

Cyanide

Cyanide

$p, p^{\prime}-D D D$

$p, p^{\prime}-D D D$

$p, p^{\prime}-D D E$

$p, p^{\prime}-D D E$

$p, p^{\prime}-D D T$

$p, p^{\prime}$-DDT

Dibenz $[a, h]$ anthracene

Dibromochloromethane

Di-n-butyl phthalate

1,2-Dichlorobenzene

1,3-Dichlorobenzene

1,4-Dichlorobenzene

3, 3'-Dichlorobenzidine

Dichlorodifluoromethane

1,1-Dichloroethane

1,2-Dichloroethane

1,1-Dichloroethylene

trans-1,2-Dichloroethylene

Dichloromethane (Methylene chloride)

2,4-Dichlorophenol

1,2-Dichloropropane

cis-1,3-Dichloropropene

trans-1,3-Dichloropropene

Dieldrin

Dieldrin

Diethyl phthalate

2,4-Dimethyl phenol

Dimethyl phthalate

2,4-Dinitrophenol

2,4-Dinitrotoluene

2,6-Dinitrotoluene

Di-n-octyl phthalate

1,2-Diphenylhydrazine

Endosulfan I

Endosulfan I

Endosulfan II
Result

$<1.0$

$<0.50$

$<0.50$

3,890

3,950

$<1.0$

$<10$

$<1.0$

$<1.0$

$<1.0$

$<1.0$

$<1.0$

$<10$

$<10$

$<10$

$<4.0$

$<10$

$<4.0$

$<5.0$

$<5.0$

$<0.10$

$<0.10$

$<0.10$

$<0.10$

$<0.10$

$<0.10$

$<10$

$<1.0$

$<10$

$<1.0$

$<1.0$

$<1.0$

$<10$

$<1.0$

$<1.0$

$<1.0$

$<1.0$

$<1.0$

$<1.0$

$<10$

$<1.0$

$<1.0$

$<1.0$

$<0.50$

$<0.50$

$<10$

$<10$

$<10$

$<45$

$<10$

$<10$

$<10$

$<10$

$<0.10$

$<0.10$

$<0.10$
Unit Flag Lab

$\mu \mathrm{g} / \mathrm{L} \quad \mathrm{O} \quad \mathrm{GE}$

$\mu g / L \quad 0 \quad$ GE

$\mu g / L \quad 0 \quad$ GE

$\mu g / L \quad 0 \quad G E$

$\mu g / L \quad 0 \quad G E$

$\mu \mathrm{g} / \mathrm{L} \quad \mathrm{O} \quad \mathrm{GE}$

$\mu g / L \quad 0 \quad$ GE

$\mu a / L \quad O \quad$ GE

$\begin{array}{lll}\mu_{\mathrm{g}} / \mathrm{L} & 0 & \mathrm{GE} \\ \mu_{\mathrm{g}} / \mathrm{L} & 0 & \mathrm{GE}\end{array}$

$\mu \mathrm{g} / \mathrm{L} \quad \mathrm{O} \quad \mathrm{GE}$

$\mu g / L \quad O \quad G E$

$\mu g / L \quad 0 \quad$ GE

$\mu \mathrm{g} / \mathrm{L} \quad \mathrm{O} \quad \mathrm{GE}$

$\mu \mathrm{g} / \mathrm{L} \quad \mathrm{O} \quad \mathrm{GE}$

$\mu \mathrm{g} / \mathrm{L} \quad 0 \quad \mathrm{GE}$

$\mu \mathrm{g} / \mathrm{L} \quad \mathrm{O} \quad \mathrm{GE}$

$\mu g / L \quad O \quad$ GE

$\mu \mathrm{g} / \mathrm{L} \quad \mathrm{O} \quad \mathrm{GE}$

$\mu \mathrm{g} / \mathrm{L} \quad \mathrm{O} \quad \mathrm{GE}$

$\mu \mathrm{g} / \mathrm{L} \quad \mathrm{O} \quad \mathrm{GE}$

$\mu g / L \quad O \quad G E$

$\mu \mathrm{g} / \mathrm{L} \quad \mathrm{O} \quad \mathrm{GE}$

$\mu \mathrm{g} / \mathrm{L} \quad \mathrm{O} \quad \mathrm{GE}$

$\mu \mathrm{g} / \mathrm{L} \quad \mathrm{O} \quad \mathrm{GE}$

$\mu g / L \quad 0 \quad G E$

$\mu \mathrm{g} / \mathrm{L} \quad \mathrm{O} \quad \mathrm{GE}$

$\mu \mathrm{g} / \mathrm{L} \quad \mathrm{O} \quad \mathrm{GE}$

$\mu g / L \quad O \quad G E$

$\mu \mathrm{g} / \mathrm{L} \quad 0 \quad \mathrm{GE}$

$\mu \mathrm{g} / \mathrm{L} \quad \mathrm{O} \quad \mathrm{GE}$

$\mu \mathrm{g} / \mathrm{L} \quad \mathrm{O} \quad \mathrm{GE}$

$\mu g / L \quad 0 \quad G E$

$\mu \mathrm{g} / \mathrm{L} \quad \mathrm{O} \quad \mathrm{GE}$

$\mu g / L \quad 0 \quad$ GE

$\mu g / L \quad 0 \quad$ GE

$\mu \mathrm{g} / \mathrm{L} \quad 0 \quad \mathrm{GE}$

$\mu \mathrm{g} / \mathrm{L} \quad \mathrm{O} \quad \mathrm{GE}$

$\mu \mathrm{g} / \mathrm{L} \quad \mathrm{O} \quad \mathrm{GE}$

$\mu g / L \quad 0 \quad G E$

$\mu \mathrm{g} / \mathrm{L} \quad \mathrm{O} \quad \mathrm{GE}$

$\mu \mathrm{g} / \mathrm{L} \quad 0$

$\mu \mathrm{g} / \mathrm{L} \quad 0 \quad \mathrm{GE}$

GE

$\mu \mathrm{g} / \mathrm{L} \quad 0 \quad \mathrm{GE}$

$\mu \mathrm{g} / \mathrm{L} \quad 0$

$\mu \mathrm{g} / \mathrm{L} \quad 0 \quad \mathrm{GE}$

$\mu g / L \quad O \quad G E$

$\mu g / L \quad 0 \quad G E$

$\mu \mathrm{g} / \mathrm{L} \quad \mathrm{O} \quad \mathrm{GE}$

$\mu \mathrm{g} / \mathrm{L} \quad \mathrm{O} \quad \mathrm{GE}$

$\mu \mathrm{g} / \mathrm{L} \quad 0 \quad \mathrm{GE}$

$\mu \mathrm{g} / \mathrm{L} \quad 0 \quad \mathrm{GE}$

$\mu \mathrm{g} / \mathrm{L} \quad 0 \quad \mathrm{GE}$

$\mu g / L \quad 0 \quad$ GE

= exceeded holding time. $=$ exceeded primary drinking water standard. 
WELL AMB 4B collected on 04/28/92, laboratory analyses (cont.)

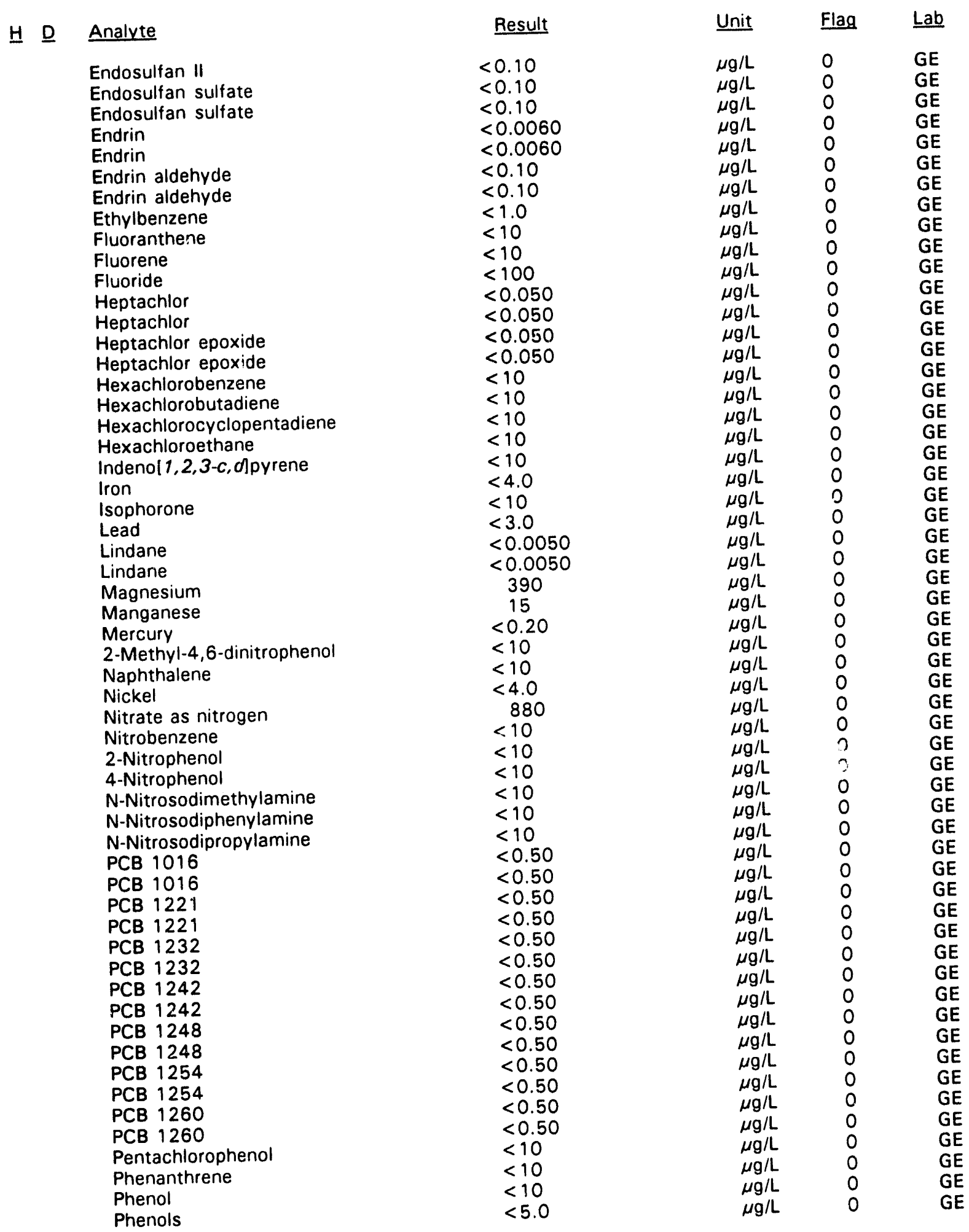

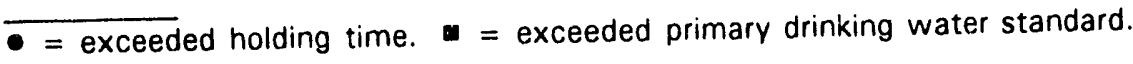


WELL AMB 4B collected on 04/28/92, laboratory analyses (cont.)

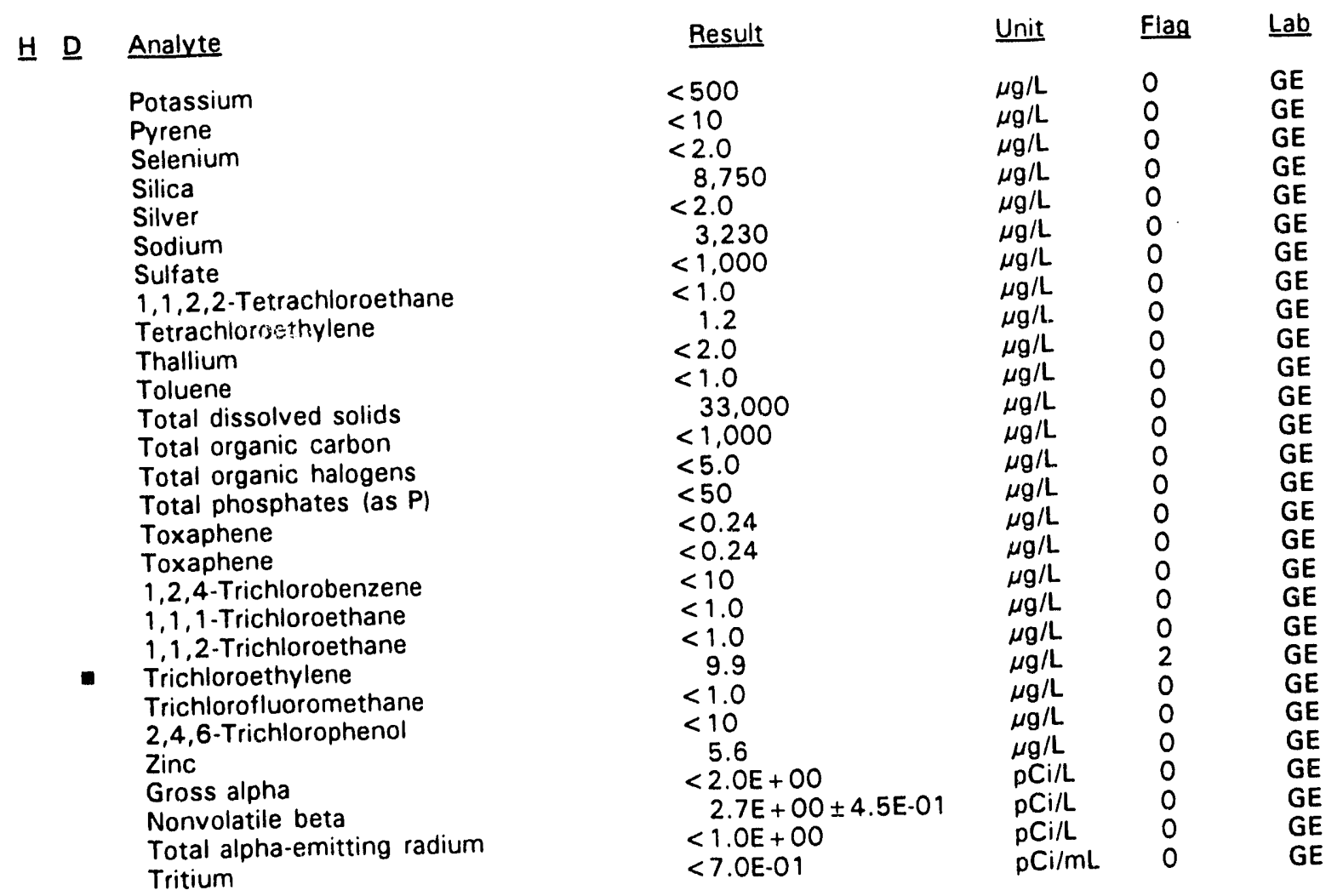

WELL AMB 4D

\begin{tabular}{|c|c|c|c|c|c|}
\hline SRS Coord. & Lat/Longitude & Screen Zone Elevation & Top of Casing & Casing & Formation \\
\hline $\begin{array}{l}\text { N104154.7 } \\
\text { E51489.0 }\end{array}$ & $\begin{array}{l}33.340124^{\circ} \mathrm{N} \\
81.733584^{\circ} \mathrm{W}\end{array}$ & $4-213.4 \mathrm{ft} \mathrm{msl}$ & $380.3 \mathrm{ft} \mathrm{msl}$ & 4" PVC & able $\left(I I B_{2}\right)$ \\
\hline
\end{tabular}

MEASUREMENTS CONDUCTED IN THE FIELD

Sample date: $04 / 28 / 92$

Depth to water: $146.91 \mathrm{ft}(44.78 \mathrm{~m})$ below TOC

Time: $14: 20$

$\mathrm{pH}: 5.5$

Alkalinity: $9 \mathrm{mg} / \mathrm{L}$

Water elevation: $233.39 \mathrm{ft}(71.14 \mathrm{~m}) \mathrm{ms}$

Water temperature: $18.0^{\circ} \mathrm{C}$

Sp. conductance: $44 \mu \mathrm{S} / \mathrm{cm}$
Water evacuated before sampling: $52 \mathrm{gal}$

\section{LABORATORY ANALYSES}

$\begin{array}{llllll}\text { H D Analyte } & \text { Result } & \underline{\text { Unit }} & \underline{\text { Flag }} & \underline{\text { Lab }} \\ \text { - Asbestos } & <0.15 & \mathrm{MSL} & 0 & \mathrm{SP} \\ \mathrm{pH} & 5.9 & \mathrm{pH} & 0 & \mathrm{GE} \\ \text { Priority Pollutant Dioxin Screen } & \mathrm{N} & \mathrm{Y} / \mathrm{N} & 0 & \mathrm{GE} \\ \text { Specific conductance } & 40 & \mu \mathrm{S} / \mathrm{cm} & 0 & \mathrm{GE} \\ \text { Acenaphthene } & <10 & \mu \mathrm{L} / \mathrm{L} & 0 & \mathrm{GE} \\ \text { Acenaphthylene } & <10 & \mu \mathrm{g} / \mathrm{L} & 0 . & \mathrm{GE}\end{array}$

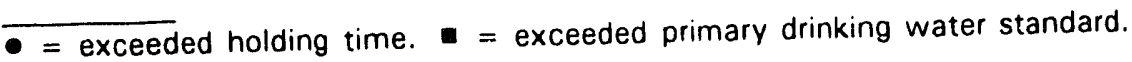


WELL AMB 4D collected on 04/28/92, laboratory analyses (cont.)

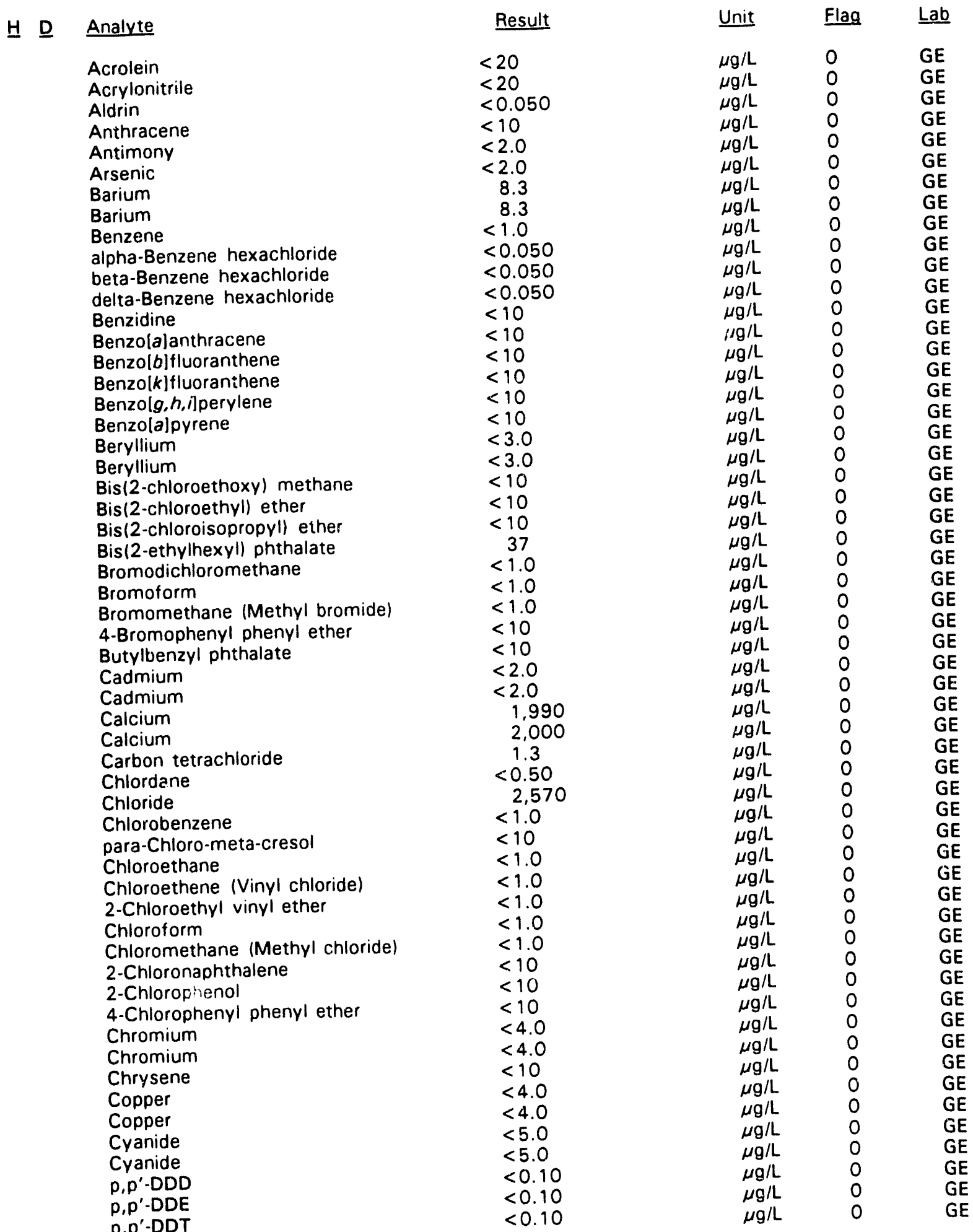

- =xceeded holding time. $\quad$ = exceeded primary drinking water standard.

Metallurgical Laboratory HWMF D-14

Second Quarter 1992 
WELL AMB 4D collected on 04/28/92, laboratory analyses (cont.)

H $\underline{\text { Analyte }}$

Dibenzla,hlanthracene

Dibromochloromethane

Di-n-butyl phthalate

1,2-Dichlorobenzene

1,3-Dichlorobenzene

1.4-Dichlorobenzene

3,3'-Dichlorobenzidine

Dichlorodifluoromethane

1,1-Dichloroethane

1,2-Dichloroethane

1,1-Dinhloroethylene

trans-1,2-Dichloroethylene

Dichloromethane (Methylene chloride)

2,4-Cichlorophenol

1,2 Dichloropropane

cis-1,3-Dichloropropene

trans-1,3-Dichloropropene

Dieldrin

Diethyl phthalate

2,4-Dimethyl phenol

Dimethyl phthalate

2,4-Dinitrophenol

2,4-Dinitrotoluene

2,6-Dinitrotoluene

Di-n-octyl phthalate

1,2-Diphenylhydrazine

Endosulfan I

Endosulfan II

Endosulfan sulfate

Endrin

Endrin aldehyde

Ethylbenzene

Fluoranthene

Fluorene

Fluoride

Fluoride

Heptachlor

Heptachlor epoxide

Hexachlorobenzene

Hexachlorobutadiene

Hexachlorocyclopentadiene

Hexachloroethane

Indenol 1,2,3-c, d]pyrene

Iron

Iron

Isophorone

Lead

Lindane

Magnesium

Magnesium

Manganese

Manganese

Mercury

2-Methyi-4,6-dinitrophenol

Naphthalene

Nickel

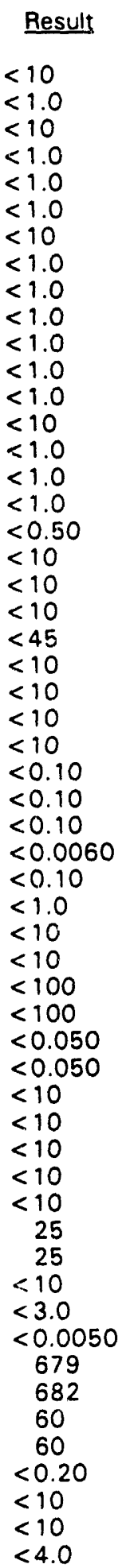

\begin{tabular}{|c|c|c|}
\hline Unit & Flag & Lab \\
\hline$\mu \mathrm{g} / \mathrm{L}$ & 0 & GE \\
\hline$\mu \mathrm{g} / \mathrm{L}$ & 0 & GE \\
\hline$\mu \mathrm{g} / \mathrm{L}$ & 0 & GE \\
\hline$\mu \mathrm{g} / \mathrm{L}$ & 0 & GE \\
\hline$\mu \mathrm{g} / \mathrm{L}$ & 0 & GE \\
\hline$\mu \mathrm{g} / \mathrm{L}$ & 0 & GE \\
\hline$\mu \mathrm{g} / \mathrm{L}$ & 0 & GE \\
\hline$\mu g / L$ & 0 & $\mathrm{GE}$ \\
\hline$\mu g / L$ & 0 & GE \\
\hline$\mu \mathrm{g} / \mathrm{L}$ & 0 & GE \\
\hline$\mu g / L$ & 0 & GE \\
\hline$\mu g / L$ & 0 & GE \\
\hline$\mu g / L$ & 0 & GE \\
\hline$\mu g / L$ & 0 & GE \\
\hline$\mu \mathrm{g} / \mathrm{L}$ & 0 & GE \\
\hline$\mu \mathrm{g} / \mathrm{L}$ & 0 & GE \\
\hline$\mu g / L$ & 0 & GE \\
\hline$\mu \mathrm{g} / \mathrm{L}$ & 0 & GE \\
\hline$\mu \mathrm{g} / \mathrm{L}$ & 0 & GE \\
\hline$\mu \mathrm{g} / \mathrm{L}$ & c & GE \\
\hline$\mu \mathrm{g} / \mathrm{L}$ & 0 & GE \\
\hline$\mu g / L$ & 0 & GE \\
\hline$\mu g / L$ & 0 & GE \\
\hline$\mu \mathrm{g} / \mathrm{L}$ & 0 & GE \\
\hline$\mu g / L$ & 0 & GE \\
\hline$\mu g / L$ & 0 & GE \\
\hline$\mu g / L$ & 0 & GE \\
\hline$\mu \mathrm{g} / \mathrm{L}$ & 0 & GE \\
\hline$\mu \mathrm{g} / \mathrm{L}$ & 0 & GE \\
\hline$\mu g / L$ & 0 & GE \\
\hline$\mu g / L$ & 0 & GE \\
\hline$\mu g / L$ & 0 & GE \\
\hline$\mu g / L$ & 0 & GE \\
\hline$\mu \mathrm{g} / \mathrm{L}$ & 0 & GE \\
\hline$\mu g / L$ & 0 & GE \\
\hline$\mu g / L$ & 0 & $\mathrm{GE}$ \\
\hline$\mu \mathrm{g} / \mathrm{L}$ & 0 & GE \\
\hline$\mu g / L$ & 0 & GE \\
\hline$\mu g / L$ & 0 & GE \\
\hline$\mu g / L$ & 0 & GE \\
\hline$\mu g / L$ & 0 & GE \\
\hline$\mu g / L$ & 0 & GE \\
\hline$\mu \mathrm{g} / \mathrm{L}$ & 0 & JE \\
\hline$\mu g / L$ & 0 & GE \\
\hline$\mu g / L$ & 0 & GE \\
\hline$\mu \mathrm{g} / \mathrm{L}$ & 0 & $\mathrm{GE}$ \\
\hline$\mu \mathrm{g} / \mathrm{L}$ & 0 & GE \\
\hline$\mu \mathrm{g} / \mathrm{L}$ & 0 & GE \\
\hline$\mu g / L$ & 0 & GE \\
\hline$\mu g / L$ & 0 & GE \\
\hline$\mu g / L$ & 2 & GE \\
\hline$\mu \mathrm{g} / \mathrm{L}$ & 2 & GE \\
\hline$\mu \mathrm{g} / \mathrm{L}$ & 0 & GE \\
\hline$\mu \mathrm{g} / \mathrm{L}$ & 0 & GE \\
\hline$g / L$ & 0 & GE \\
\hline$g / L$ & 0 & $F$ \\
\hline
\end{tabular}

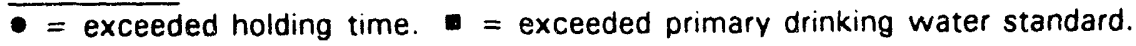


WELL AMB 4D collected on 04/28/92, laboratory analyses (cont.)

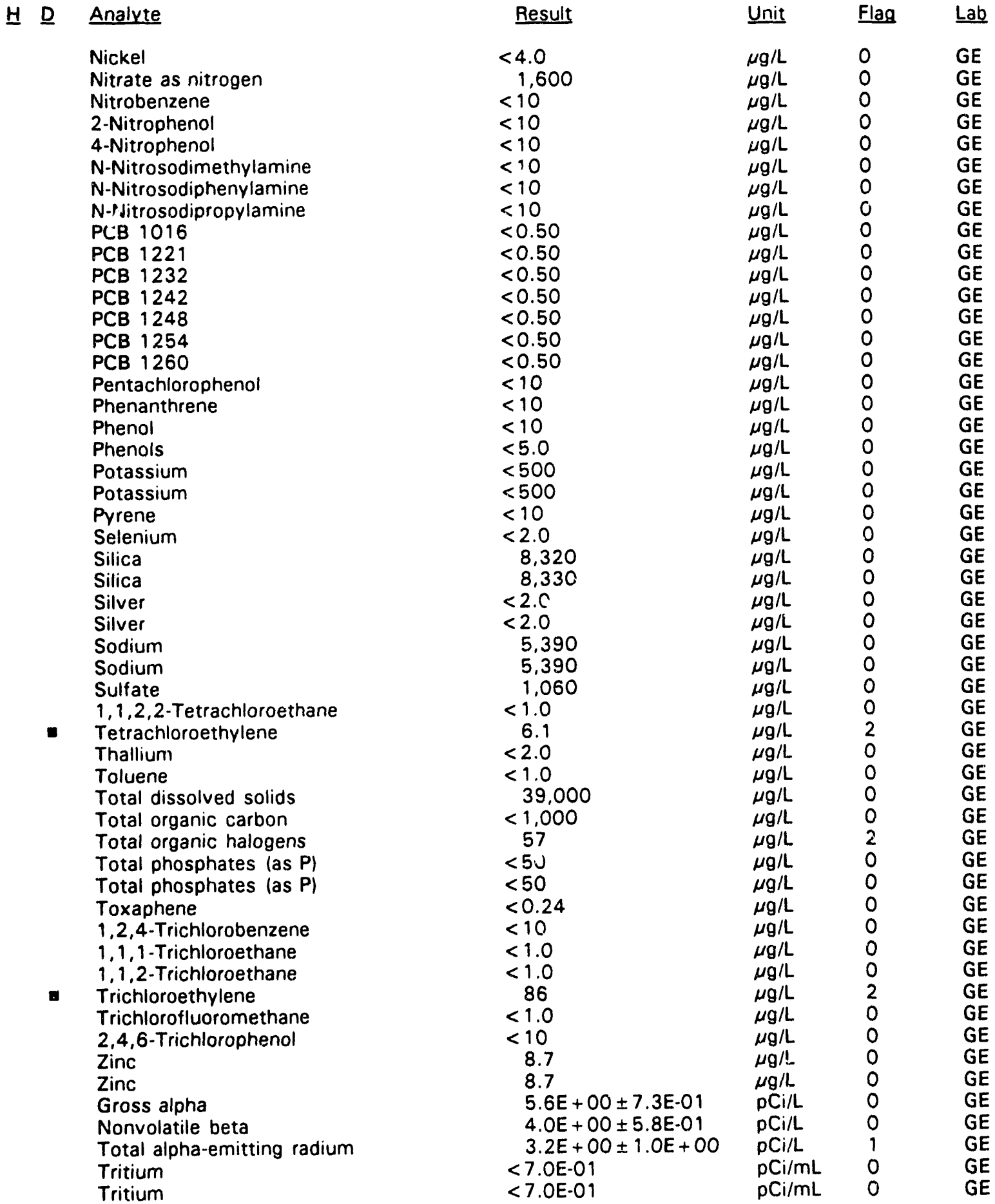

$\overline{-=\text { exceeded holding time. }}=$ exceeded primary drinking water standard. 
WELL AMB 5

\begin{tabular}{|c|c|c|c|c|c|}
\hline SRS Coord. & Lat/Longitude & Screen Zone Elevation & Top of Casing & Casing & Formation \\
\hline $\begin{array}{l}N 104083.4 \\
E 51467.2\end{array}$ & $\begin{array}{l}33.339930^{\circ} \mathrm{N} \\
81.733502^{\circ} \mathrm{W}\end{array}$ & $242.1-222.1 \mathrm{ft} \mathrm{msl}$ & $379.6 \mathrm{ft} \mathrm{msl}$ & 4" PVC & Water Table $\left(\| \mid B_{2}\right)$ \\
\hline
\end{tabular}

\section{MEASUREMENTS CONDUCTED IN THE FIELD}

Sample date: $04 / 28 / 92$

Depth to water: $145.84 \mathrm{ft}(44.45 \mathrm{~m})$ below TOC

Water elevation: $233.76 \mathrm{ft}(71.25 \mathrm{~m}) \mathrm{msl}$

Sp. conductance: $44 \mu \mathrm{S} / \mathrm{cm}$

Water evacuated before sampling: $31 \mathrm{gal}$

Time: $15: 15$

pH: 4.7

Alkalinity: $1 \mathrm{mg} / \mathrm{L}$

Water temperature: $18.0^{\circ} \mathrm{C}$

\section{LABORATORY ANALYSES}

H D Analyte

Carbon tetrachloride

Chloroform

Iron

Lead

Magnesium

Nickel

- Tetrachioroethylene

Total organic carbon

Total organic halogens

1,1,1-Trichloroethane

- Trichloroethylene

- Total alpha-emitting radium
Result

$$
2.2
$$

1.8

14

$<3.0$

598

$<4.0$

8.3

$<1,000$

75

$<1.0$

150

$6.3 E+00 \pm 1.4 E+00$
Unit

$\mu \mathrm{g} / \mathrm{L}$

$\mu \mathrm{g} / \mathrm{L}$

$\mu g / L$

$\mu g / L$

$\mu \mathrm{g} / \mathrm{L}$

$\mu \mathrm{g} / \mathrm{L}$

$\mu \mathrm{g} / \mathrm{L}$

$\mu g / L$

$\mu g / L$

$\mu \mathrm{g} / \mathrm{L}$

$\mu g / L$

$\mathrm{pCi/L}$

$\begin{array}{ll}\text { Flag } & \text { Lab } \\ 0 & \text { GE } \\ 0 & \text { GE } \\ 0 & \text { GE } \\ 0 & \text { GE } \\ 0 & \text { GE } \\ 0 & \text { GE } \\ 2 & \text { GE } \\ 0 & \text { GE } \\ 2 & \text { GE } \\ 0 & \text { GE } \\ 2 & \text { GE } \\ 2 & \text { GE }\end{array}$

WELL AMB 6

\begin{tabular}{|c|c|c|c|c|c|}
\hline SRS Coord. & Lat/Longitude & Screen Zone Elevation & Top of Casing & Casing & Formation \\
\hline $\begin{array}{l}N 104034.1 \\
E 51466.0\end{array}$ & $\begin{array}{l}33.339819^{\circ} \mathrm{N} \\
81.733410^{\circ} \mathrm{W}\end{array}$ & $242.6-222.6 \mathrm{ft} \mathrm{msl}$ & $377.2 \mathrm{ft} \mathrm{msl}$ & 4" PVC & Water Table $\left(\| \mathrm{B}_{2}\right)$ \\
\hline
\end{tabular}

\section{MEASUREMENTS CONDUCTED IN THE FIELD}

Sample date: $05 / 11 / 92$

Depth to water: $143.37 \mathrm{ft}(43.70 \mathrm{~m})$ below TOC

Water elevation: $233.83 \mathrm{ft}(71.27 \mathrm{~m}) \mathrm{msl}$

Sp. conductance: $34 \mu \mathrm{S} / \mathrm{cm}$

Water evacuated before sampling: $29 \mathrm{gal}$

\section{LABORATORY ANALYSES}

H D Analyte

- Carbon tetrachloride
Chloroform
Iron
Lead
Magnesium

$\overline{0 \text { exceeded holding time. }} \mathbf{-}=$ exceeded primary drinkir.g water standard.
Time: $11: 45$

pH: 5.1

Alkalinity: $7 \mathrm{mg} / \mathrm{L}$

Water temperature: $20.0^{\circ} \mathrm{C}$

\begin{tabular}{|c|c|}
\hline Unit & Flag \\
\hline $\begin{array}{l}\mu g / L \\
\mu g / L \\
\mu g / L \\
\mu g / L \\
\mu g / L\end{array}$ & $\begin{array}{l}0 \\
0 \\
0 \\
0 \\
0\end{array}$ \\
\hline
\end{tabular}


WELL AMB 6 collected on 05/11/92, laboratory analyses (cont.)

\begin{tabular}{|c|c|c|c|c|}
\hline$\underline{H} \underline{D}$ & Analyte & Result & Unit & Flag \\
\hline$\bullet$ & $\begin{array}{l}\text { Nickel } \\
\text { Tetrachloroethylene } \\
\text { Total organic carbon } \\
\text { Total organic halogens } \\
1,1,1 \text {-Trichloroethane } \\
\text { Trichloroethylene } \\
\text { Total alpha-emitting radium }\end{array}$ & $\begin{aligned}< & 4.0 \\
< & 1.0 \\
< & 1.000 \\
< & 5.0 \\
< & 1.0 \\
& 4.9 \\
& 2.0 E+00 \pm 1.2 E+00\end{aligned}$ & $\begin{array}{l}\mu \mathrm{g} / \mathrm{L} \\
\mu \mathrm{g} / \mathrm{L} \\
\mu \mathrm{g} / \mathrm{L} \\
\mu \mathrm{g} / \mathrm{L} \\
\mu \mathrm{g} / \mathrm{L} \\
\mu \mathrm{g} / \mathrm{L} \\
\mathrm{pCi} / \mathrm{L}\end{array}$ & $\begin{array}{l}0 \\
0 \\
0 \\
0 \\
0 \\
1 \\
0\end{array}$ \\
\hline
\end{tabular}

\section{WELL AMB 7}

\begin{tabular}{|c|c|c|c|c|c|}
\hline SRS coord. & Lat/Longitude & Screen Zone Elevation & Top of Casing & Casing & Formation \\
\hline $\begin{array}{l}\text { N103920.0 } \\
\text { E5 1624.9 }\end{array}$ & $\begin{array}{l}33.339827{ }^{\circ} \mathrm{N} \\
81.732769{ }^{\circ} \mathrm{W}\end{array}$ & $242.1-222.1 \mathrm{ft} \mathrm{msl}$ & $369.9 \mathrm{ft} \mathrm{msl}$ & 4" PVC & le $\left(\| B_{2}\right)$ \\
\hline
\end{tabular}

\section{MEASUREMENTS CONDUCTED IN THE FIELD}

Sample date: 05/12/92

Depth to water: $135.77 \mathrm{ft}(41.38 \mathrm{~m})$ below TOC

Water elevation: $234.13 \mathrm{ft}(71.36 \mathrm{~m} / \mathrm{msl}$

Sp. conductance: $91 \mu \mathrm{S} / \mathrm{cm}$

Water evacuated before sampling: $6 \mathrm{gal}$

The well went dry during purging.

\section{LABORATORY ANALYSES}

H D $\quad$ Analyte
- Carbon tetrachloride
Chloroform
Iron
Lead
Magnesium
Nickel
Tetrachloroethylene
Total organic carbon
Total organic halogens
1,1,1-Trichloroethane
Trichloroethylene
Total alpha-emitting radium

Time: $11: 20$

$\mathrm{pH}: 6.1$

Alkalinity: $37 \mathrm{mg} / \mathrm{L}$

Water temperature: $21.3^{\circ} \mathrm{C}$

$\begin{array}{cccc}\text { Result } & \text { Unit } & \text { Flag } & \text { Lab } \\ <1.0 & \mu \mathrm{g} / \mathrm{L} & 0 & \mathrm{GE} \\ <1.0 & \mu \mathrm{g} / \mathrm{L} & 0 & \mathrm{GE} \\ 118 & \mu \mathrm{g} / \mathrm{L} & 0 & \mathrm{GE} \\ <3.0 & \mu \mathrm{g} / \mathrm{L} & 0 & \mathrm{GE} \\ 271 & \mu \mathrm{g} / \mathrm{L} & 0 & \mathrm{GE} \\ 8.5 & \mu \mathrm{g} / \mathrm{L} & 0 & \mathrm{GE} \\ <1.0 & \mu \mathrm{g} / \mathrm{L} & 0 & \mathrm{GE} \\ <1.000 & \mu \mathrm{g} / \mathrm{L} & 0 & \mathrm{GE} \\ 12 & \mu \mathrm{g} / \mathrm{L} & 0 & \mathrm{GE} \\ <1.0 & \mu \mathrm{g} / \mathrm{L} & 0 & \mathrm{GE} \\ 3.6 & \mu \mathrm{g} / \mathrm{L} & 1 & \mathrm{GE} \\ <1.0 \mathrm{G}+00 & \mathrm{pCi} / \mathrm{L} & 0 & \mathrm{GE}\end{array}$

- exceeded holding time. = exceeded primary drinking water standard. 
WELL AMB 7A

\begin{tabular}{|c|c|c|c|c|c|}
\hline SRS Coord. & Lat/Longitude & Screen Zone Elevation & Top of Casing & Casing & Formation \\
\hline $\begin{array}{l}\text { N103987.1 } \\
\text { E51591.0 }\end{array}$ & $\begin{array}{l}33.339920^{\circ} \mathrm{N} \\
81.732989^{\circ} \mathrm{W}\end{array}$ & $125.6-115.6 \mathrm{ft} \mathrm{msl}$ & $373.6 \mathrm{ft} \mathrm{msl}$ & 4" PVC & (IIA) \\
\hline
\end{tabular}

MEASUREMENTS CONDUCTED IN THE FIELD

Sample date: 05/12/92

Depth to water: $154.10 \mathrm{ft}(46.97 \mathrm{~m})$ below TOC

Water elevation: $219.50 \mathrm{ft}(66.90 \mathrm{~m}) \mathrm{msl}$

Sp. conductance: $43 \mu \mathrm{S} / \mathrm{cm}$

Water evacuated before sampling: $273 \mathrm{gal}$

Time: 10:50

$\mathrm{pH}: 5.8$

Alkalinity: $13 \mathrm{mg} / \mathrm{L}$

Water temperature: $19.8^{\circ} \mathrm{C}$

\section{LABORATORY ANALYSES}

\section{H D Analyte}

Asbestos

Asbestos

$\mathrm{pH}$

$\mathrm{pH}$

Priority Pollutant Dioxin Screen

Specific conductance

Specific conductance

Acenaphthene

Acenaphthylene

- Acrolein

- Acrylonitrile

Aldrin

Anthraceme

Antimony

Arsenic

Barium

Barium

- Benzene

alpha-Benzene hexachloride

beta-Benzene hexachloride

delta-Benzene hexachloride

Benzidine

Benzolalanthracene

Benzolb]fluoranthene

Benzolk]fluoranthene

Benzolg, $h, i]$ perylene

Benzolajpyrene

Beryllium

Beryllium

Bis(2-chloroethoxy) methane

Bis(2-chloroethyl) ether

Bis (2-chloroisopropyl) ether

Bis(2-ethylhexyl) phthalate

Bromodichloromethane

Bromoform

Bromomethane (Methyl bromide)

4-Bromophenyl phenyl ether

Butylbenzyl phthalate

Cadmium

$\begin{array}{rl} & \text { Result } \\ & =0.15 \\ < & 0.15 \\ & 6.9 \\ & 6.8 \\ & N \\ & 45 \\ 45 & 4 \\ < & 10 \\ < & 10 \\ < & 20 \\ <20 \\ <0.050 \\ <10 \\ <2.0 \\ <2.0 \\ \quad 6.2 \\ 6.1 \\ <1.0 \\ <0.050 \\ <0.050 \\ <0.050 \\ <10 \\ <10 \\ <10 \\ <10 \\ <10 \\ <10 \\ <10 \\ <10 \\ <3.0 \\ <3.0 \\ <10 \\ <10 \\ <10 \\ <10 \\ <10 \\ <1.0 \\ <1.0 \\ <1.0 \\ <10 \\ <10 \\ <2.0 \\ <\end{array}$

Result

Unit Flag Lab

MSL $\quad 0 \quad S P$

MSL $\quad 0 \quad S P$

$\mathrm{pH} \quad \mathrm{O} \quad \mathrm{GE}$

$\mathrm{pH} \quad \mathrm{O} \quad \mathrm{GE}$

$Y / N \quad 0 \quad G E$

$\mu \mathrm{S} / \mathrm{cm} \quad \mathrm{O} \quad \mathrm{GE}$

$\mu S / c m$ GE

$\mu \mathrm{g} / \mathrm{L} \quad \mathrm{O} \quad \mathrm{GE}$

$\mu g / L \quad O \quad G E$

$\mu \mathrm{g} / \mathrm{L} \quad \mathrm{O} \quad \mathrm{GE}$

$\mu g / L \quad 0 \quad G E$

$\mu g / L \quad 0 \quad G E$

$\mu \mathrm{g} / \mathrm{L} \quad \mathrm{O} \quad \mathrm{GE}$

$\mu g / L \quad O \quad G E$

$\mu \mathrm{g} / \mathrm{L} \quad \mathrm{O} \quad \mathrm{GE}$

$\mu g / L \quad 0 \quad G E$

$\mu \mathrm{g} / \mathrm{L} \quad \mathrm{O} \quad \mathrm{GE}$

$\mu \mathrm{g} / \mathrm{L} \quad \mathrm{O} \quad \mathrm{GE}$

$\mu \mathrm{g} / \mathrm{L} \quad \mathrm{O} \quad \mathrm{GE}$

$\mu g / L \quad 0 \quad G E$

$\mu \mathrm{g} / \mathrm{L} \quad \mathrm{O} \quad \mathrm{GE}$

$\mu \mathrm{g} / \mathrm{L} \quad \mathrm{D} \quad \mathrm{GE}$

$\mu g / L \quad 0 \quad$ GE

$\mu \mathrm{g} / \mathrm{L} \quad \mathrm{O} \quad \mathrm{GE}$

$\mu g / L \quad 0 \quad$ GE

$\mu g / L \quad 0 \quad$ GE

$\mu \mathrm{g} / \mathrm{L} \quad \mathrm{O} \quad \mathrm{GE}$

$\mu g / L \quad 0 \quad G E$

$\mu g / L \quad 0 \quad G E$

$\mu \mathrm{g} / \mathrm{L} \quad \mathrm{O} \quad \mathrm{GE}$

$\mu g / L \quad 0 \quad$ GE

$\mu g / L \quad 0 \quad$ GE

$\mu \mathrm{g} / \mathrm{L} \quad 0 \quad \mathrm{GE}$

$\mu \mathrm{g} / \mathrm{L} \quad \mathrm{O} \quad \mathrm{GE}$

$\mu \mathrm{g} / \mathrm{L} . \quad 0 \quad \mathrm{GE}$

$\mu \mathrm{g} / \mathrm{L} \quad 0 \quad \mathrm{GE}$

$\mu g / L \quad 0 \quad$ GE

$\mu \mathrm{g} / \mathrm{L} \quad \mathrm{O} \quad \mathrm{GE}$

$\mu g / L$

GE

- = exceeded holding time. - =xceeded primary drinking water standard. 
WELL AMB 7A collected on 05/12/92, laboratory analyses (cont.)

\begin{tabular}{|c|c|c|c|c|c|}
\hline \multicolumn{6}{|c|}{ Result } \\
\hline $\begin{array}{l}\bullet \\
\bullet \\
\bullet \\
\bullet \\
\bullet \\
\bullet \\
\bullet \\
\bullet \\
\bullet\end{array}$ & 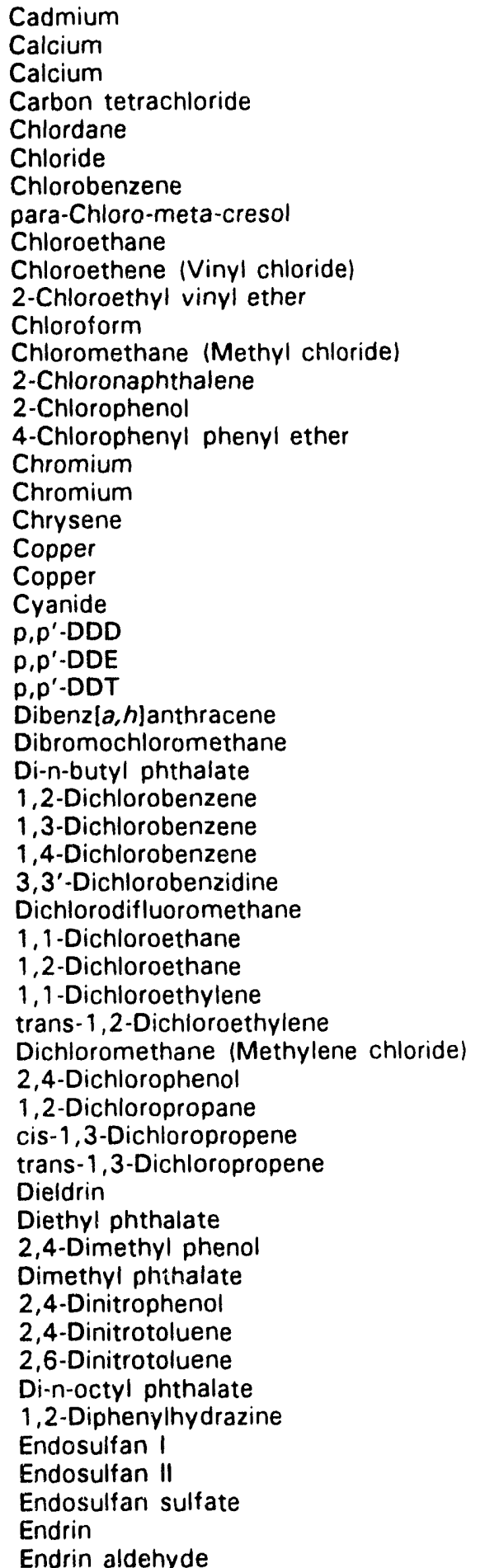 & $\begin{array}{rl}< & 2.0 \\
& 4.890 \\
& 4.820 \\
< & 1.0 \\
<0.50 & 1.760 \\
& <1.0 \\
< & 10 \\
<1.0 \\
<1.0 \\
<1.0 \\
<1.0 \\
<1.0 \\
<10 \\
<10 \\
<10 \\
<4.0 \\
<4.0 \\
<10 \\
<1.0 \\
<4.0 \\
<5.0 \\
<0.10 \\
<0.10 \\
<0.10 \\
<10 \\
<1.0 \\
<10 \\
<10 \\
<1.0 \\
<1.0 \\
<1.0 \\
<10 \\
<1.0 \\
<1.0 \\
<1.0 \\
<1.0 \\
<1.0 \\
<1.1 \\
<10 \\
<1.0 \\
<1.0 \\
<1.0 \\
<0.50 \\
<10 \\
<10 \\
<10 \\
<45 \\
<10 \\
<10 \\
<10 \\
<10 \\
<0.10 \\
<0.10 \\
<0.10 \\
<0.0060 \\
<0.10 \\
<10\end{array}$ & $\begin{array}{l}\mu \mathrm{g} / \mathrm{L} \\
\mu \mathrm{g} / \mathrm{L} \\
\mu \mathrm{g} / \mathrm{L} \\
\mu \mathrm{g} / \mathrm{L} \\
\mu \mathrm{g} / \mathrm{L} \\
\mu \mathrm{g} / \mathrm{L} \\
\mu \mathrm{g} / \mathrm{L} \\
\mu \mathrm{g} / \mathrm{L} \\
\mu \mathrm{g} / \mathrm{L} \\
\mu \mathrm{g} / \mathrm{L} \\
\mu \mathrm{g} / \mathrm{L} \\
\mu \mathrm{g} / \mathrm{L} \\
\mu \mathrm{g} / \mathrm{L} \\
\mu \mathrm{g} / \mathrm{L} \\
\mu \mathrm{g} / \mathrm{L} \\
\mu \mathrm{g} / \mathrm{L} \\
\mu \mathrm{g} / \mathrm{L} \\
\mu \mathrm{g} / \mathrm{L} \\
\mu \mathrm{g} / \mathrm{L} \\
\mu \mathrm{g} / \mathrm{L} \\
\mu \mathrm{g} / \mathrm{L} \\
\mu \mathrm{g} / \mathrm{L} \\
\mu \mathrm{g} / \mathrm{L} \\
\mu \mathrm{g} / \mathrm{L} \\
\mu \mathrm{g} / \mathrm{L} \\
\mu \mathrm{g} / \mathrm{L} \\
\mu \mathrm{g} / \mathrm{L} \\
\mu \mathrm{g} / \mathrm{L} \\
\mu \mathrm{g} / \mathrm{L} \\
\mu \mathrm{g} / \mathrm{L} \\
\mu \mathrm{g} / \mathrm{L} \\
\mu \mathrm{g} / \mathrm{L} \\
\mu \mathrm{g} / \mathrm{L} \\
\mu \mathrm{g} / \mathrm{L} \\
\mu \mathrm{g} / \mathrm{L} \\
\mu \mathrm{g} / \mathrm{L} \\
\mu \mathrm{g} / \mathrm{L} \\
\mu \mathrm{g} / \mathrm{L} \\
\mu \mathrm{g} / \mathrm{L} \\
\mu \mathrm{g} / \mathrm{L} \\
\mu \mathrm{g} / \mathrm{L} \\
\mu \mathrm{g} / \mathrm{L} \\
\mu \mathrm{g} / \mathrm{L} \\
\mu \mathrm{g} / \mathrm{L} \\
\mu \mathrm{g} / \mathrm{L} \\
\mu \mathrm{g} / \mathrm{L} \\
\mu \mathrm{g} / \mathrm{L} \\
\mu \mathrm{g} / \mathrm{L} \\
\mu \mathrm{g} / \mathrm{L} \\
\mu \mathrm{g} / \mathrm{L} \\
\mu \mathrm{g} / \mathrm{L} \\
\mu \mathrm{g} / \mathrm{L} \\
\mu \mathrm{g} / \mathrm{L} \\
\mu \mathrm{g} / \mathrm{L} \\
\mu \mathrm{g} / \mathrm{L} \\
\mu \mathrm{g} / \mathrm{L}\end{array}$ & $\begin{array}{l}0 \\
0 \\
0 \\
0 \\
0 \\
0 \\
0 \\
0 \\
0 \\
0 \\
0 \\
0 \\
0 \\
0 \\
0 \\
0 \\
0 \\
0 \\
0 \\
0 \\
0 \\
0 \\
0 \\
0 \\
0 \\
0 \\
0 \\
0 \\
0 \\
0 \\
0 \\
0 \\
0 \\
0 \\
0 \\
0 \\
0 \\
0 \\
0 \\
0 \\
0 \\
0 \\
0 \\
0 \\
0 \\
0 \\
0 \\
0 \\
0 \\
0 \\
0 \\
0 \\
0 \\
0 \\
0 \\
0\end{array}$ & $\begin{array}{l}\mathrm{GE} \\
\mathrm{GE} \\
\mathrm{GE} \\
\mathrm{GE} \\
\mathrm{GE} \\
\mathrm{GE} \\
\mathrm{GE} \\
\mathrm{GE} \\
\mathrm{GE} \\
\mathrm{GE} \\
\mathrm{GE} \\
\mathrm{GE} \\
\mathrm{GE} \\
\mathrm{GE} \\
\mathrm{GE} \\
\mathrm{GE} \\
\mathrm{GE} \\
\mathrm{GE} \\
\mathrm{GE} \\
\mathrm{GE} \\
\mathrm{GE} \\
\mathrm{GE} \\
\mathrm{GE} \\
\mathrm{GE} \\
\mathrm{GE} \\
\mathrm{GE} \\
\mathrm{GE} \\
\mathrm{GE} \\
\mathrm{GE} \\
\mathrm{GE} \\
\mathrm{GE} \\
\mathrm{GE} \\
\mathrm{GE} \\
\mathrm{GE} \\
\mathrm{GE} \\
\mathrm{GE} \\
\mathrm{GE} \\
\mathrm{GE} \\
\mathrm{GE} \\
\mathrm{GE} \\
\mathrm{GE} \\
\mathrm{GE} \\
\mathrm{GE} \\
\mathrm{GE} \\
\mathrm{GE} \\
\mathrm{GE} \\
\mathrm{GE} \\
\mathrm{GE} \\
\mathrm{GE} \\
\mathrm{GE} \\
\mathrm{GE} \\
\mathrm{GE} \\
\mathrm{GE} \\
\mathrm{GE} \\
\mathrm{GE} \\
\mathrm{GE}\end{array}$ \\
\hline
\end{tabular}

- exceeded holding time. - exceeded primary drinking water standard. 
WELL AMB 7A collected on 05/12/92, laboratory analyses (cont.)

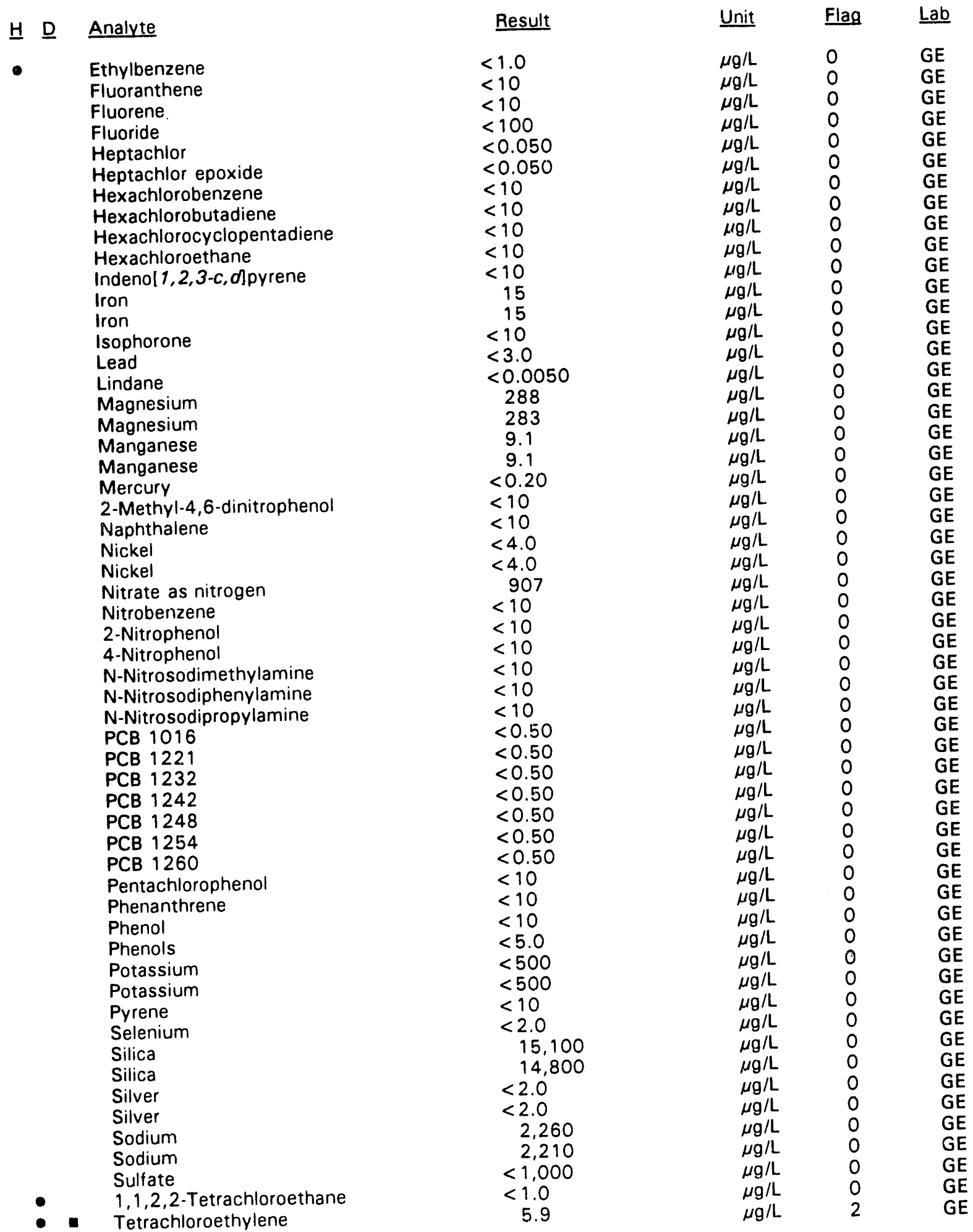

- exceeded holding time. $\mathbf{\square}=$ exceeded primary drinking water standard. 
WELL AMB 7A collected on 05/12/92, laboratory analyses (cont.)

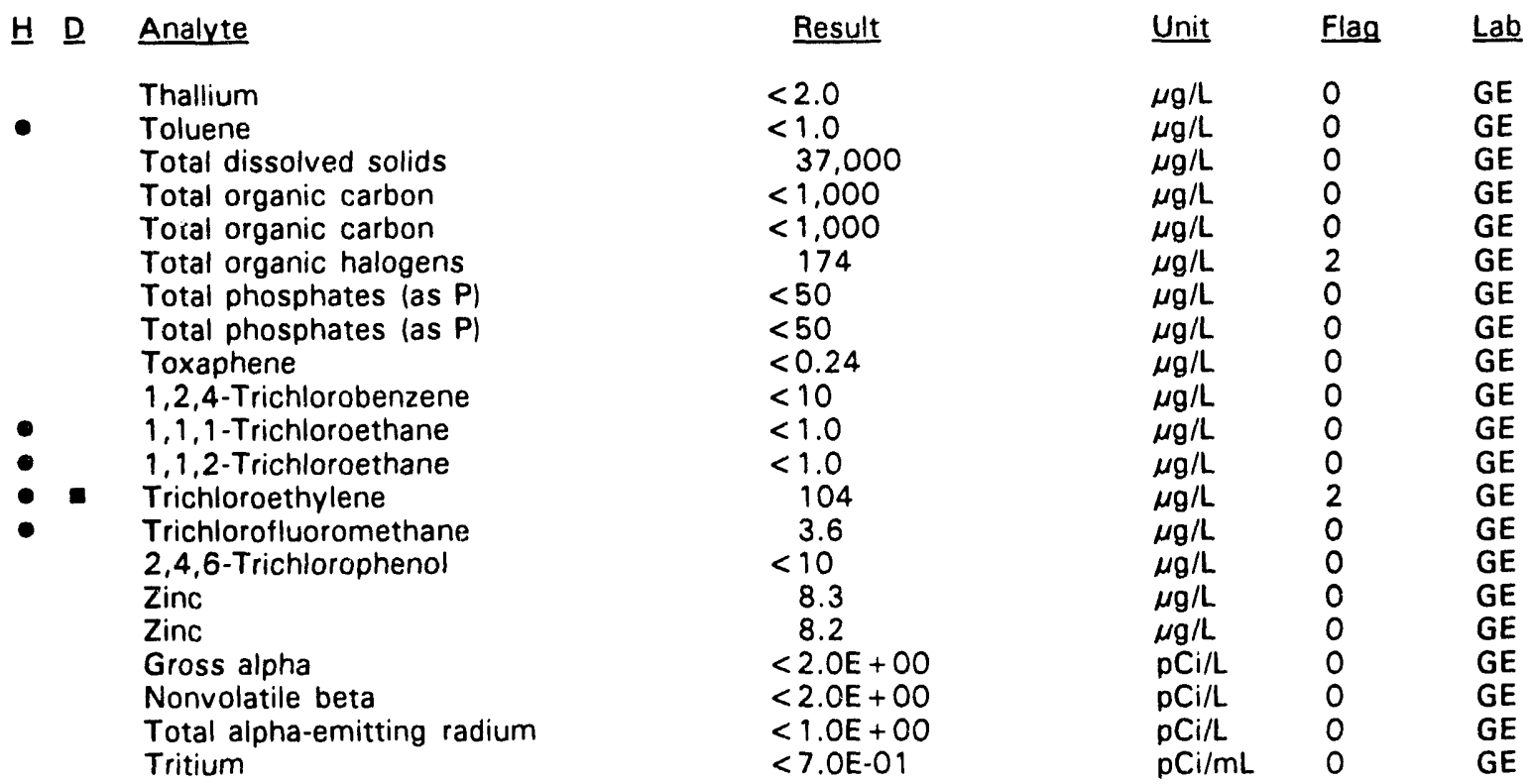

\section{WELL AMB 7B}

\begin{tabular}{|c|c|c|c|c|c|}
\hline SRS Coord. & Lat/Longitude & Screen Zone Elevation & Top of Casing & Casing & Formation \\
\hline 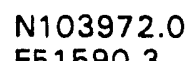 & 5 o & $a \mathrm{ft} \mathrm{mal}$ & 37 & 4" PVC & \\
\hline
\end{tabular}

\section{MEASUREMENTS CONDUCTED IN THE FIELD}

Sample date: $05 / 11 / 92$

Depth to water: $148.33 \mathrm{ft}(45.21 \mathrm{~m})$ below TOC

Water elevation: $224.67 \mathrm{ft}(68.48 \mathrm{~m}) \mathrm{msl}$

Sp. conductance: $34 \mu \mathrm{S} / \mathrm{cm}$

Water evacuated before sampling: $188 \mathrm{gal}$

\section{LABORATORY ANALYSES}

브 Analyte

Asbestos

pH

Priority Polluti : Dioxin Screen

Specific conductance

Acenaphthene

Acenaphthylene

Acrolein

Acrolein

Acrylonitrile

Acrylonitrile

Aldrin

Aldrin

Anthracene
Time: $12: 45$

$\mathrm{pH}: 4.9$

Alkalinity: $3 \mathrm{mg} / \mathrm{L}$

Water temperature: $20.9^{\circ} \mathrm{C}$

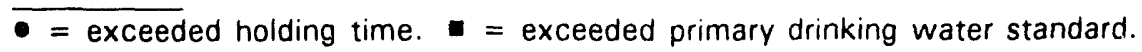

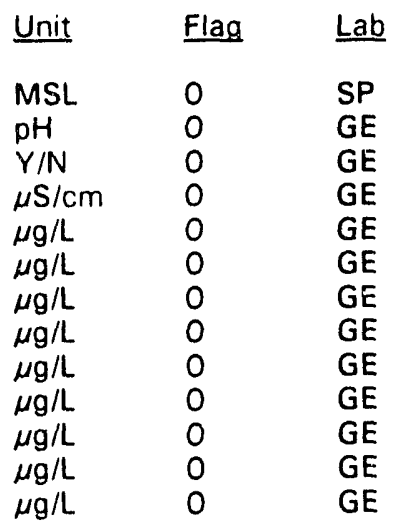

$\begin{array}{rl}< & 0.15 \\ & 5.4 \\ N & N \\ & 30 \\ < & 10 \\ <10 \\ <20 \\ <20 \\ <20 \\ <20 \\ <0.050 \\ <0.050 \\ <10\end{array}$


WELL AMB $7 B$ collected on 05/11/92, laboratory analyses (cont.)

므 Analyte

Antimony

Arsenic

Barium

Barium

Benzene

Benzene

alpha-Benzene hexachloride

alpha-Benzene hexachloride

beta-Benzene hexachloride

beta-Benzene hexachloride

delta-Benzene hexachloride

delta-Benzene hexachloride

Benzidine

Benzolalanthracerie

Benzolb]fluoranthere

Benzolk]fluoranthene

Benzolg,h,i]perylente

Berizola]pyrene

Beryllium

Beryllium

Bis(2-chloroethoxy) methane

Bis (2-chloroethyl) ether

Bis(2-chloroisopropyl) ether

Bis(2-ethyihexyl) phthalate

Bromodichloromethane

Bromodichioromethane

Bromcform

Bromoform

Bromomethane (Methyl bromide)

Bromomethane (Methyl bromide)

4-Bromophenyl phenyl ether

Butylbenzyl phthalate

Cadmium

Cadmium

Calcium

Calcium

Carbon tetrachloride

Carbon tetrachloride

Chlordane

Chlordane

Chloride

Chlorobenzene

Chlorobenzene

para-Chloro-meta-cresol

Chloroethane

Chloroethane

Chloroethene (Vinyl chloride)

Chloroethene (Vinyl chloride)

2-Chloroethyl vinyl ether

2-Chloroethyl vinyl ether

Chloroform

Chloroform

Chloromethane (Methyl chloride)

Chloromethane (Methyl chloride)

2-Chloronaphthalene

2-Chlorophenol
Result

$<2.0$

$<2.0$

$<3.0$

$<3.0$

$<1.0$

$<1.0$

$<0.050$

$<0.050$

$<0.050$

$<0.050$

$<0.050$

$<0.050$

$<10$

$<10$

$<10$

$<10$

$<10$

$<10$

$<3.0$

$<3.0$

$<10$

$<10$

$<10$

$<10$

$<1.0$

$<1.0$

$<1.0$

$<1.0$

$<1.0$

$<1.0$

$<10$

$<10$

$<2.0$

$<2.0$

251

253

$<1.0$

$<1.0$

$<0.50$

$<0.50$

3,670

$<1.0$

$<1.0$

$<10$

$<1.0$

$<1.0$

$<1.0$

$<1.0$

$<1.0$

$<1.0$

$<1.0$

$<1.0$

$<1.0$

$<1.0$

$<10$

$<10$
Unit Flag $\underline{\text { Lab }}$

$\mu g / L \quad 0 \quad G E$

$\mu \mathrm{g} / \mathrm{L} \quad 0 \quad \mathrm{GE}$

$\mu \mathrm{g} / \mathrm{L} \quad 0 \quad \mathrm{GE}$

$\mu g / L \quad 0 \quad \mathrm{GE}$

$\mu \mathrm{g} / \mathrm{L} \quad 0 \quad \mathrm{GE}$

$\mu g / L \quad 0 \quad G E$

$\mu g / L \quad 0 \quad G E$

$\mu g / L \quad 0 \quad G E$

$\mu \mathrm{g} / \mathrm{L} \quad 0 \quad \mathrm{GE}$

$\mu g / L \quad 0 \quad G E$

$\mu g / L \quad 0 \quad G E$

$\mu g / L \quad 0 \quad G E$

$\mu \mathrm{g} / \mathrm{L} \quad \mathrm{O} \quad \mathrm{GE}$

$\mu g / L \quad 0 \quad$ GE

$\mu g / L \quad 0 \quad$ GE

$\mu g / L \quad 0 \quad$ GE

$\mu g i L \quad O \quad$ GE

$\mu \mathrm{g} / \mathrm{L} \quad 0 \quad \mathrm{GE}$

$\mu \mathrm{g} / \mathrm{L} \quad 0 \quad \mathrm{GE}$

$\mu g / L \quad 0 \quad G E$

$\mu g / L \quad 0 \quad G E$

$\mu g / L \quad 0 \quad G E$

$\mu g / L \quad 0 \quad G E$

$\mu \mathrm{g} / \mathrm{L} \quad \mathrm{O} \quad \mathrm{GE}$

$\mu g / L \quad 0 \quad$ GE

$\mu \mathrm{g} / \mathrm{L} \quad \mathrm{O} \quad \mathrm{GE}$

$\mu \mathrm{g} / \mathrm{L} \quad 0 \quad \mathrm{GE}$

$\mu g / L \quad 0 \quad G E$

$\mu \mathrm{g} / \mathrm{L} \quad 0 \quad \mathrm{GE}$

$\mu g / L \quad 0 \quad G E$

$\mu g / L \quad 0 \quad G E$

$\mu g / L \quad 0 \quad$ GE

$\mu g / L \quad 0 \quad G E$

$\mu g / L \quad 0 \quad G E$

$\mu g / L \quad 0 \quad G E$

$\mu \mathrm{g} / \mathrm{L} \quad 0 \quad \mathrm{GE}$

$\mu g / L \quad 0 \quad G E$

$\mu g / L \quad 0 \quad G E$

$\mu \mathrm{g} / \mathrm{L} \quad 0 \quad \mathrm{GE}$

$\mu g / L \quad 0 \quad G E$

$\mu \mathrm{g} / \mathrm{L} \quad 0 \quad \mathrm{GE}$

$\mu \mathrm{g} / \mathrm{L} \quad 0 \quad \mathrm{GE}$

$\mu \mathrm{g} / \mathrm{L} \quad 0 \quad \mathrm{GE}$

GE

$\mu g / L \quad 0 \quad G E$

GE

$\mu \mathrm{g} / \mathrm{L} \quad \mathrm{O} \quad \mathrm{GE}$

$\mu \mathrm{g} / \mathrm{L} \quad 0 \quad \mathrm{GE}$

$\mu g / L \quad 0 \quad G E$

$\mu \mathrm{g} / \mathrm{L} \quad 0 \quad \mathrm{GE}$

$\mu g / L \quad 0 \quad G E$

$\mu \mathrm{g} / \mathrm{L} \quad 0$

$\mu \mathrm{g} / \mathrm{L} \quad 0 \quad \mathrm{GE}$

$\mu g / L \quad 0$

$\mu \mathrm{g} / \mathrm{L} \quad \mathrm{O} \quad \mathrm{GE}$

$\mu \mathrm{g} / \mathrm{L}$

- =xceeded holding time. = exceeded primary drinking water standard. 
WELL AMB 7B collected on 05/11/92, laboratory analyses (cont.)

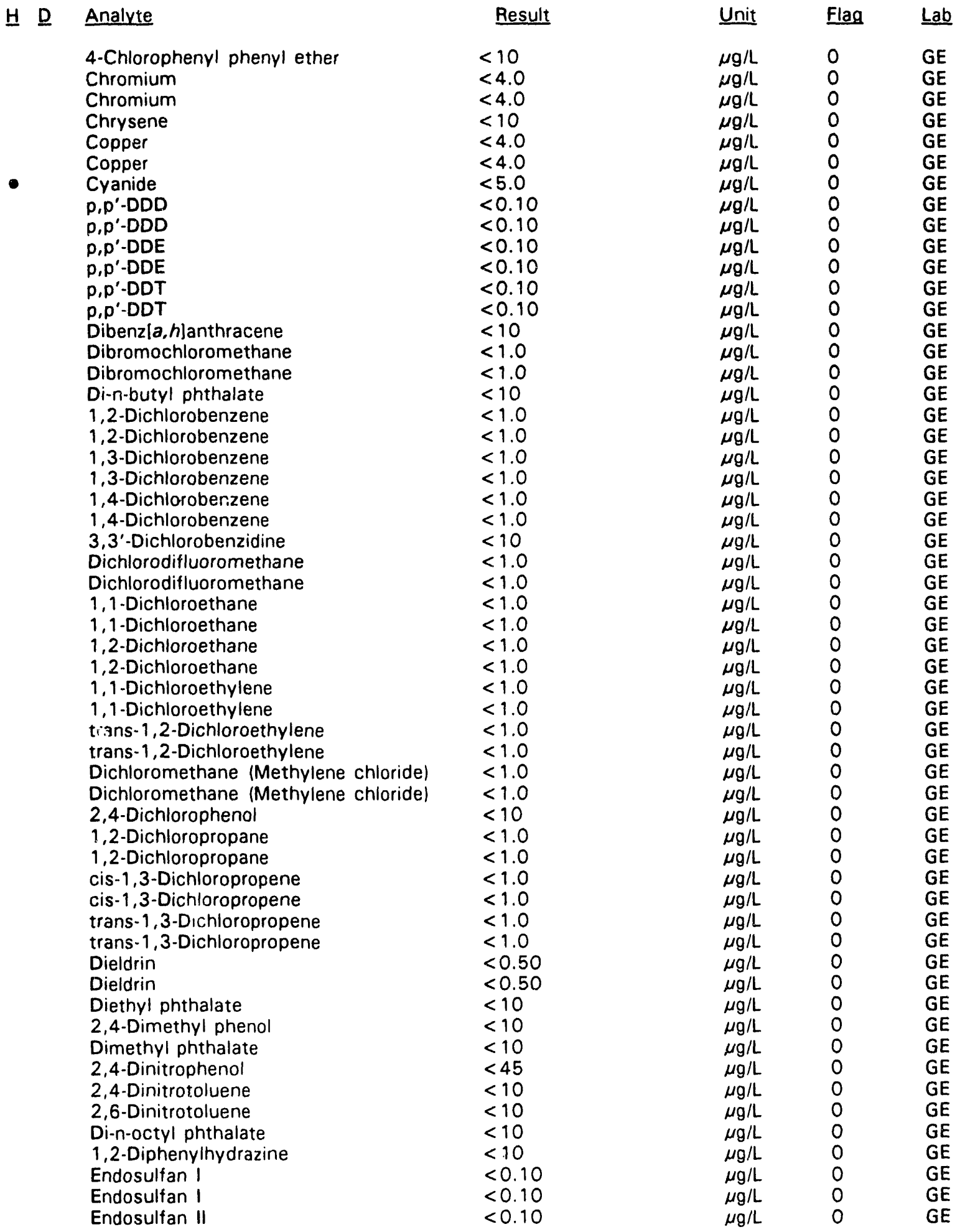

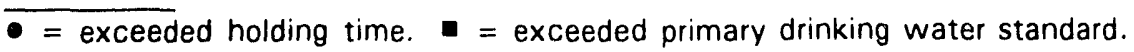


WELL AMB 7B collected on 05/11/92, laboratory analyses (cont.)

H D Analyte

Endosulfan II

Endosulfan sulfate

Endosulfan sulfate

Endrin

Endrin

Endrin aldehyde

Endrin aldehyde

Ethylbenzene

Ethylbenzene

Fluoranthene

Fluorene

Fluoride

Heptachlor

Heptachlor

Heptachlor epoxide

Heptachlor epoxide

Hexachlorobenzene

Hexachlorobutadiene

Hexachlorocyclopentadiene

Hexachloroethane

Indenol 1,2,3-c, d)pyrene

Iron

Iron

Isophorone

Lead

Lindane

Lindane

Magnesium

Magnesium

Manganese

Manganese

Mercury

2-Methyl-4,6-dinitrophenol

Naphthalene

Nickel

Nickel

Nitrate as nitrogen

Nitrobenzene

2-Nitrophenol

4-Nitrophenol

N-Nitrosodimethylamine

$\mathrm{N}$-Nitrosodiphenylamine

N-Nitrosodipropylamine

PCB 1016

PCB 1016

PCB 1221

PCB 1221

PCB 1232

PCB $: 232$

PCB 1242

PCB 1242

PCB 1248

PCB 1248

PCB 1254

PCB 1254

PCB 1260

\section{Result}

$<0.10$

$<0.10$

$<0.10$

$<0.0060$

$<0.0060$

$<0.10$

$<0.10$

$<1.0$

$<1.0$

$<10$

$<10$

$<100$

$<0.050$

$<0.050$

$<0.050$

$<0.050$

$<10$

$<10$

$<10$

$<10$

$<10$

4.9

4.9

$<10$

$<3.0$

$<0.0050$

$<0.0050$

82

82

3.9

4.0

$<0.20$

$<10$

$<10$

$<4.0$

$<4.0$

270

$<10$

$<10$

$<10$

$<10$

$<10$

$<10$

$<0.50$

$<0.50$

$<0.50$

$<0.50$

$<0.50$

$<0.50$

$<0.50$

$<0.50$

$<0.50$

$<0.50$

$<0.50$

$<0.50$

$<0.50$
Unit Flag Lab

$\mu g / L \quad 0 \quad$ GE

$\mu \mathrm{g} / \mathrm{L} \quad \mathrm{O} \quad \mathrm{GE}$

$\mu g / L \quad O \quad G E$

$\mu \mathrm{g} / \mathrm{L} \quad \mathrm{O} \quad \mathrm{GE}$

$\mu g / L \quad O \quad G E$

$\mu g / L \quad 0 \quad$ GE

$\mu g / L \quad O \quad G E$

$\mu g / L \quad 0 \quad$ GE

$\mu \mathrm{g} / \mathrm{L} \quad \mathrm{O} \quad \mathrm{GE}$

$\mu g / L \quad 0 \quad$ GE

$\mu g / L \quad 0 \quad G E$

$\mu g / L \quad 0 \quad$ GE

$\mu g / L \quad 0 \quad$ GE

$\mu \mathrm{g} / \mathrm{L} \quad \mathrm{G} \quad \mathrm{GE}$

$\mu g / L \quad O \quad$ GE

$\mu g / L \quad O \quad G E$

$\mu \mathrm{g} / \mathrm{L} \quad \mathrm{O} \quad \mathrm{GE}$

$\mu g / L \quad O \quad G E$

$\mu \mathrm{g} / \mathrm{L} \quad \mathrm{O} \quad \mathrm{GE}$

$\mu \mathrm{g} / \mathrm{L} \quad \mathrm{O} \quad \mathrm{GE}$

$\mu \mathrm{g} / \mathrm{L} \quad \mathrm{O} \quad \mathrm{GE}$

$\mu \mathrm{g} / \mathrm{L} \quad \mathrm{O} \quad \mathrm{GE}$

$\mu \mathrm{g} / \mathrm{L} \quad \mathrm{O} \quad \mathrm{GE}$

$\mu \mathrm{g} / \mathrm{L} \quad \mathrm{O} \quad \mathrm{GE}$

$\mu g / L \quad O \quad G E$

$\mu g / L \quad 0 \quad$ GE

$\mu g / L \quad O \quad G E$

$\mu \mathrm{g} / \mathrm{L} \quad \mathrm{O} \quad \mathrm{GE}$

$\mu g / L \quad O \quad G E$

$\mu g / L \quad O \quad G E$

$\mu g / L \quad O \quad G E$

$\mu \mathrm{g} / \mathrm{L} \quad \mathrm{O} \quad \mathrm{GE}$

$\mu \mathrm{g} / \mathrm{L} \quad \mathrm{O} \quad \mathrm{GE}$

$\mu \mathrm{g} / \mathrm{L} \quad 0 \quad \mathrm{GE}$

$\mu g / L \quad O \quad G E$

$\mu \mathrm{g} / \mathrm{L} \quad \mathrm{O} \quad \mathrm{GE}$

$\mu \mathrm{g} / \mathrm{L} \quad \mathrm{O} \quad \mathrm{GE}$

$\mu \mathrm{g} / \mathrm{L} \quad \mathrm{O} \quad \mathrm{GE}$

$\mu g / L \quad O \quad$ GE

$\mu g / L \quad O \quad$ GE

$\mu g / L \quad O \quad G E$

$\mu \mathrm{g} / \mathrm{L} \quad \mathrm{O} \quad \mathrm{GE}$

$\mu \mathrm{g} / \mathrm{L} \quad \mathrm{O} \quad \mathrm{GE}$

$\mu \mathrm{g} / \mathrm{L} \quad \mathrm{O} \quad \mathrm{GE}$

$\mu \mathrm{g} / \mathrm{L} \quad \mathrm{O} \quad \mathrm{GE}$

$\mu g / L \quad O \quad G E$

$\mu g / L \quad 0 \quad$ GE

$\mu \mathrm{g} / \mathrm{L} \quad \mathrm{O} \quad \mathrm{GE}$

$\mu \mathrm{g} / \mathrm{L} \quad 0 \quad \mathrm{GE}$

$\mu \mathrm{g} / \mathrm{L} \quad 0 \quad \mathrm{GE}$

$\mu \mathrm{g} / \mathrm{L} \quad \mathrm{G} \quad \mathrm{GE}$

$\mu \mathrm{g} / \mathrm{L} \quad \mathrm{O} \quad \mathrm{GE}$

$\mu \mathrm{g} / \mathrm{L} \quad 0 \quad \mathrm{GE}$

$\mu \mathrm{g} / \mathrm{L} \quad \mathrm{O} \quad \mathrm{GE}$

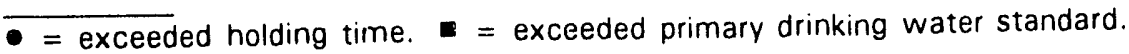


WELL AMB 7B collected on 05/11/92, laboratory analyses (cont.)

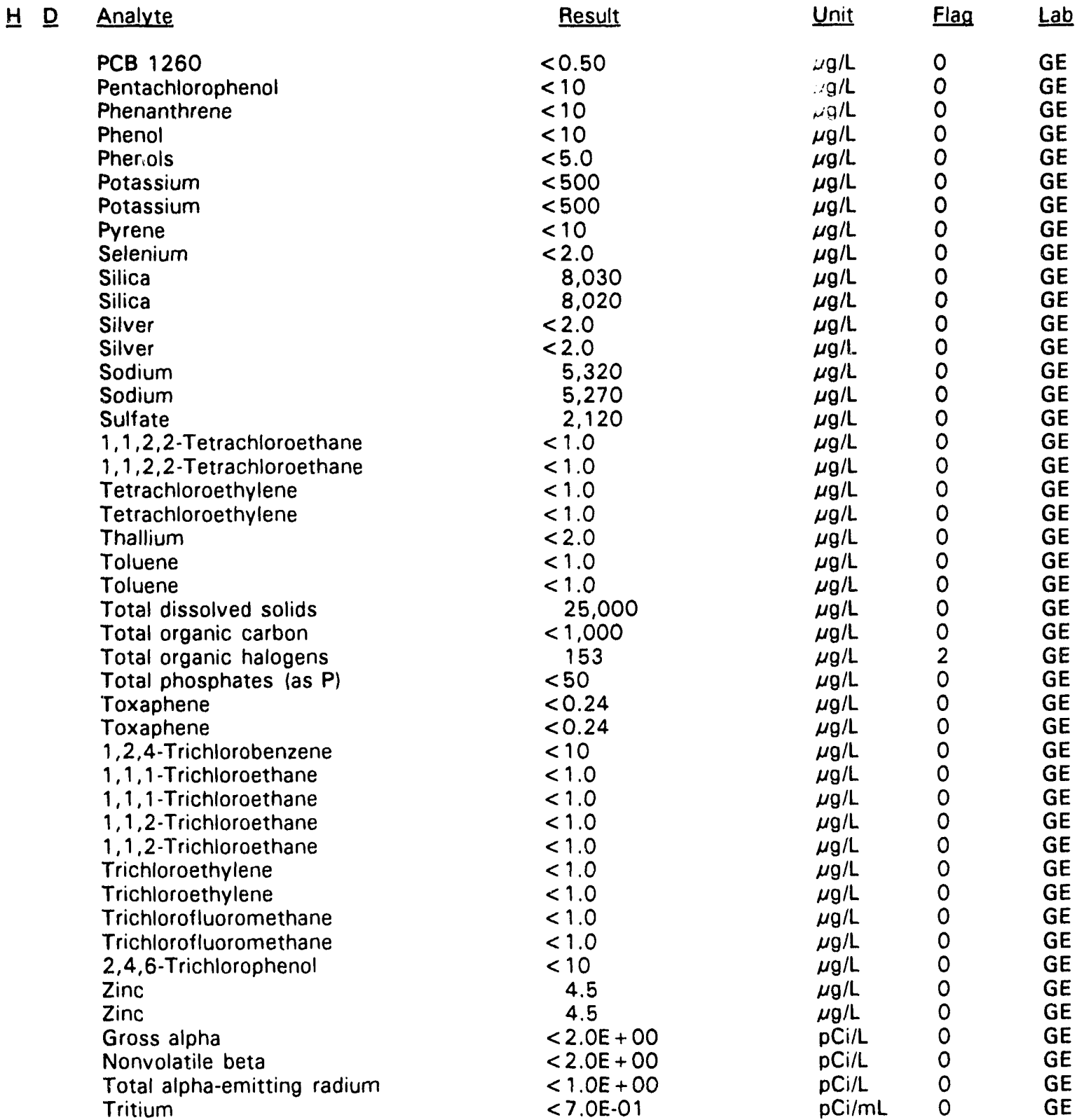

- = exceeded holding time. $=$ exceeded primary drinking water standard. 
WELL AMB 8D

\begin{tabular}{|c|c|c|c|c|c|}
\hline SRS C & Lat/Longitude & Screen Zone Elevation & Top of Casing & Casing & Formation \\
\hline $\begin{array}{l}\text { N103874.7 } \\
\text { E5 1400.5 }\end{array}$ & $\begin{array}{l}33.339360^{\circ} \mathrm{N} \\
81.733272^{\circ} \mathrm{W}\end{array}$ & $240.8-220.8 \mathrm{ft} \mathrm{msl}$ & $369.6 \mathrm{ft} \mathrm{msl}$ & 4" PVC & Water Table $\left(11 \mathrm{~B}_{2}\right)$ \\
\hline
\end{tabular}

\section{MEASUREMENTS CONDUCTED IN THE FIELD}

Sample date: $04 / 28 / 92$

Depth to water: $135.88 \mathrm{ft}(41.42 \mathrm{~m})$ below TOC

Water elevation: $233.72 \mathrm{ft}(71.24 \mathrm{~m}) \mathrm{msl}$

Sp. conductance: $62 \mu \mathrm{S} / \mathrm{cm}$

Water evacuated before sampling: $34 \mathrm{gal}$

Time: 9:50

$\mathrm{pH}: 5.7$

Alkalinity: $16 \mathrm{mg} / \mathrm{L}$

Water temperature: $17.5^{\circ} \mathrm{C}$

\section{LABORATORY ANALYSES}

H D Analyte

\begin{tabular}{|c|c|}
\hline $\begin{array}{l}\text { Carbon tetrachloride } \\
\text { Carbon tetrachloride } \\
\text { Carbon tetrachloride } \\
\text { Carbon tetrachloride } \\
\text { Carbon tetrachloride } \\
\text { Chloroform } \\
\text { Chloroform } \\
\text { Chloroform } \\
\text { Chloroform } \\
\text { Chloroform } \\
\text { Iron } \\
\text { Iron } \\
\text { Iron } \\
\text { Iron } \\
\text { Lead } \\
\text { Lead } \\
\text { Lead } \\
\text { Lead } \\
\text { Magnesium } \\
\text { Magnesium } \\
\text { Magnesium } \\
\text { Magnesium } \\
\text { Nickel } \\
\text { Nickel } \\
\text { Nickel } \\
\text { Nickel } \\
\text { Tetrachloroethylene } \\
\text { Tetrachloroethylene } \\
\text { Tetrachloroethylene } \\
\text { Tetrachloroethylene } \\
\text { Tetrachloroethylene } \\
\text { Total organic carbon } \\
\text { Total organic carbon } \\
\text { Total organic carbon } \\
\text { Total organic carbon } \\
\text { Total organic carbon } \\
\text { Total organic halogens } \\
\text { Total organic halogens } \\
\text { Total organic halogens }\end{array}$ & $\begin{aligned} &<1.0 \\
&<1.0 \\
&<1.0 \\
&<1.0 \\
&<1.0 \\
&<1.0 \\
&<1.0 \\
&<1.0 \\
&<1.0 \\
&<1.0 \\
& \\
& 5.1 \\
& 5.1 \\
& 9.9 \\
& 11 \\
&<3.0 \\
&<3.0 \\
&<2.0 \\
&<2.0 \\
& 134 \\
& 133 \\
& 145 \\
& 139 \\
&<4.0 \\
&<4.0 \\
& 3.3 \\
& 3.5 \\
&<1.0 \\
&<1.0 \\
&<1.0 \\
&<1.0 \\
&<1.0 \\
&<1.000 \\
&<1.000 \\
&<1.000 \\
& 2.090 \\
& 2.200 \\
&< 5.0 \\
&<5.0 \\
&<5.0 \\
&<\end{aligned}$ \\
\hline
\end{tabular}

Unit Flag Lab

$\mu g / L \quad O \quad G E$

$\mu g / L \quad O \quad G E$

$\mu g / L \quad O \quad$ GE

$\mu g / L \quad O \quad W A$

$\mu g / L \quad$ WA

$\mu \mathrm{g} / \mathrm{L} \quad \mathrm{O} \quad \mathrm{GE}$

$\mu g / L \quad O \quad G E$

$\mu \mathrm{g} / \mathrm{L} \quad \mathrm{O} \quad \mathrm{GE}$

$\mu g / L \quad 0 \quad$ WA

$\mu \mathrm{g} / \mathrm{L} \quad \mathrm{O} \quad$ WA

$\mu g / L \quad O \quad$ GE

$\mu g / L \quad O \quad G E$

$\mu \mathrm{g} / \mathrm{L} \quad 0 \quad$ WA

$\begin{array}{lll}\mu \mathrm{g} / \mathrm{L} & 0 & \mathrm{GE} \\ \mu \mathrm{g} / \mathrm{L} & 0 & \mathrm{G}\end{array}$

$\mu g / L \quad 0 \quad$ GE

$\mu g / L \quad O \quad$ WA

$\mu g / L \quad$ WA

$\mu \mathrm{g} / \mathrm{L} \quad \mathrm{O} \quad \mathrm{GE}$

$\mu \mathrm{g} / \mathrm{L} \quad \mathrm{O} \quad \mathrm{GE}$

$\mu \mathrm{g} / \mathrm{L} \quad 0 \quad$ WA

$\mu g / L \quad 0 \quad$ WA

$\mu \mathrm{g} / \mathrm{L} \quad 0 \quad$ GE

$\mu \mathrm{g} / \mathrm{L} \quad \mathrm{O} \quad \mathrm{GE}$

$\mu \mathrm{g} / \mathrm{L} \quad \mathrm{O} \quad$ WA

$\mu g / L \quad O \quad$ WA

$\mu \mathrm{g} / \mathrm{L} \quad 0 \quad \mathrm{GE}$

$\mu \mathrm{g} / \mathrm{L} \quad \mathrm{O} \quad \mathrm{GE}$

$\mu \mathrm{g} / \mathrm{L} \quad 0 \quad \mathrm{GE}$

$\mu \mathrm{g} / \mathrm{L} \quad \mathrm{W} \quad$ WA

$\mu \mathrm{g} / \mathrm{L} \quad 0 \quad$ WA

$\mu \mathrm{g} / \mathrm{L} \quad \mathrm{O} \quad \mathrm{GE}$

$\mu \mathrm{g} / \mathrm{L} \quad \mathrm{O} \quad \mathrm{GE}$

GE

$\mu \mathrm{g} / \mathrm{L} \quad 0 \quad$ WA

WA

$\mu g / L \quad 0 \quad$ GE

$\mu \mathrm{g} / \mathrm{L} \quad \mathrm{O} \quad \mathrm{GE}$

$\mu \mathrm{g} / \mathrm{L} \quad \mathrm{O}$ WA

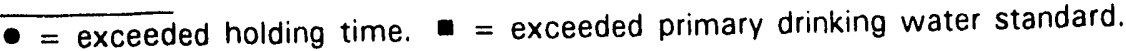


WELL AMB 8D collected on 04/28/92, laboratory analyses (cont.)

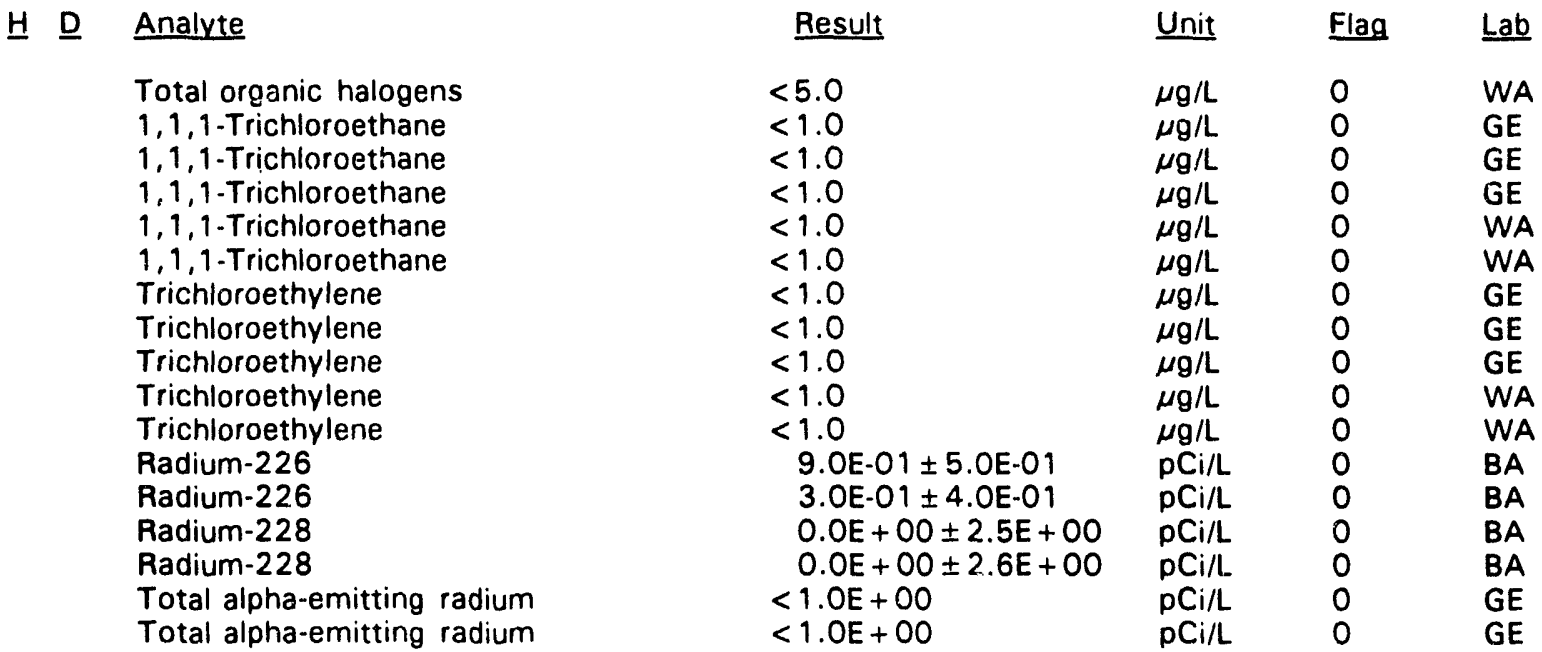

WELL AMB 9D

\begin{tabular}{|c|c|c|c|c|c|}
\hline SRS Coord. & Lat/Longitude & Screen Zone Elevation & Top of Casing & Casing & Formation \\
\hline 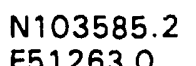 & 496 o $N$ & $9.7 \mathrm{ft} \mathrm{msl}$ & 367 & 4" PVC & Water Table $\left(11 \mathrm{~B}_{2}\right)$ \\
\hline
\end{tabular}

\section{MEASUREMENTS CONDUCTED IN THE FIELD}

Sample date: 05/11/92

Depth to water: $133.97 \mathrm{ft}(40.83 \mathrm{~m})$ below TOC

Water elevation: $233.93 \mathrm{ft}(71.30 \mathrm{~m}) \mathrm{ms}$ ।

Sp. conductance: $38, \mathrm{~N} / \mathrm{cm}$

Water evacuated before sampling: $37 \mathrm{gal}$

\section{LABORATORY ANALYSES}

$\begin{array}{ll}\text { H } & \text { Analyte } \\ \text { - } \quad \text { Carbon tetrachloride } \\ \text { Chloroform } \\ \text { Iron } \\ \text { Lead } \\ \text { Magnesium } \\ \text { Nickel } \\ \text { Tetrachloroethylene } \\ \text { Total organic carbon } \\ \text { Total organic halogens } \\ \text { 1,1,1-Trichloroethane } \\ \text { Trichloroethylene } \\ \text { Total alpha-emitting radium }\end{array}$

Time: $11: 00$

$\mathrm{pH}: 5.5$

Alkalinity: $9 \mathrm{mg} / \mathrm{L}$

Water temperature: $18.8^{\circ} \mathrm{C}$ 
WELL AMB 10A

\begin{tabular}{|c|c|c|c|c|c|}
\hline SRS Coord. & Lat/Longitude & Screen Zone Elevation & Top of Casing & Casing & Formation \\
\hline $\begin{array}{l}\text { N103326.4 } \\
\text { E51410.0 }\end{array}$ & $\begin{array}{l}33.338163^{\circ} \mathrm{N} \\
81.732180^{\circ} \mathrm{W}\end{array}$ & $111.4-106.4 \mathrm{ft} \mathrm{msl}$ & $366.5 \mathrm{ft} \mathrm{msl}$ & 4" PVC & Congaree (IIA) \\
\hline
\end{tabular}

ME^SUREMENTS CONDUCTED IN THE FIELD

Sample date: $04 / 29 / 92$

Depth to water: $148.28 \mathrm{ft}(45.20 \mathrm{~m})$ below TOC

Water elevation: $218.22 \mathrm{ft}(66.51 \mathrm{~m}) \mathrm{msl}$

Sp. conductance: $2290 \mu \mathrm{S} / \mathrm{cm}$

Water evacuated before sampli j: $46 \mathrm{gal}$

The well went dry during purging.

\section{LABORATORY ANALYSES}

H D Analyte

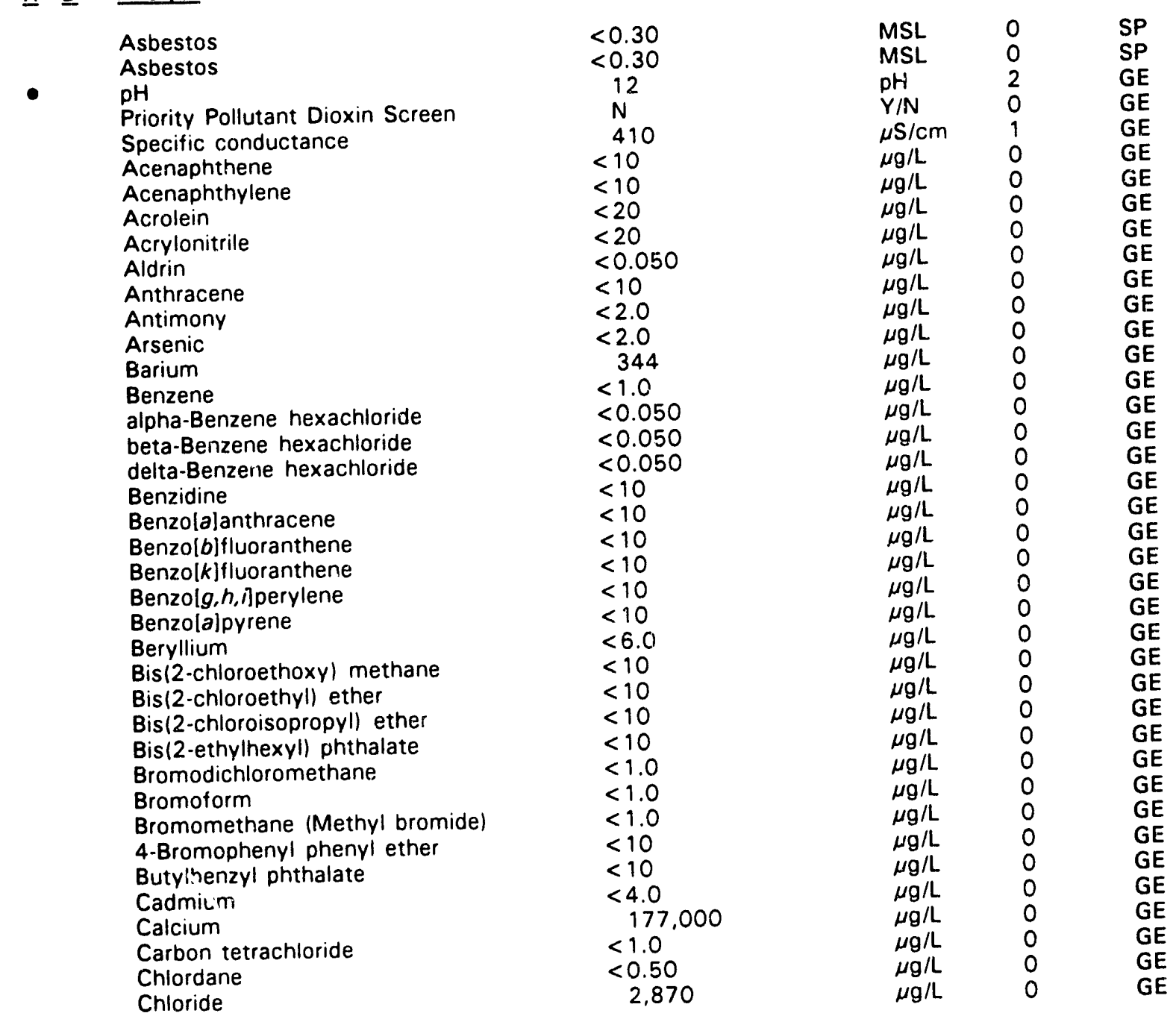

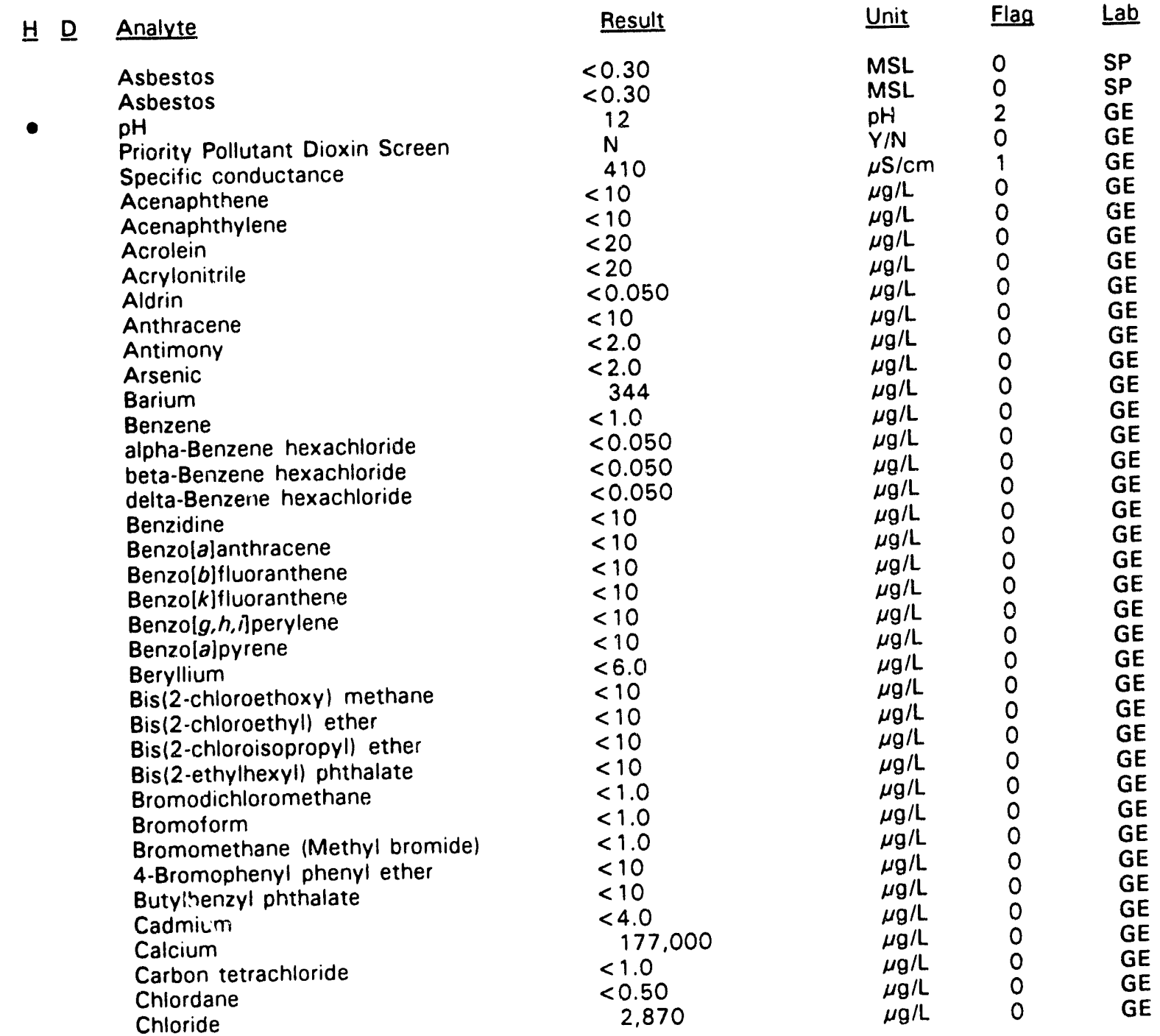

Time: $15: 30$

$\mathrm{pH}: 12.6$

Alkalinity: $519 \mathrm{mg} / \mathrm{L}$

Water temperature: $18.9^{\circ} \mathrm{C}$

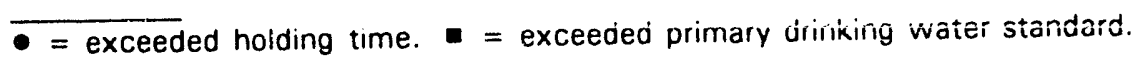


WELL AMB 1OA collected on 04/29/92, laboratory analyses (cont.)

H D Analyte

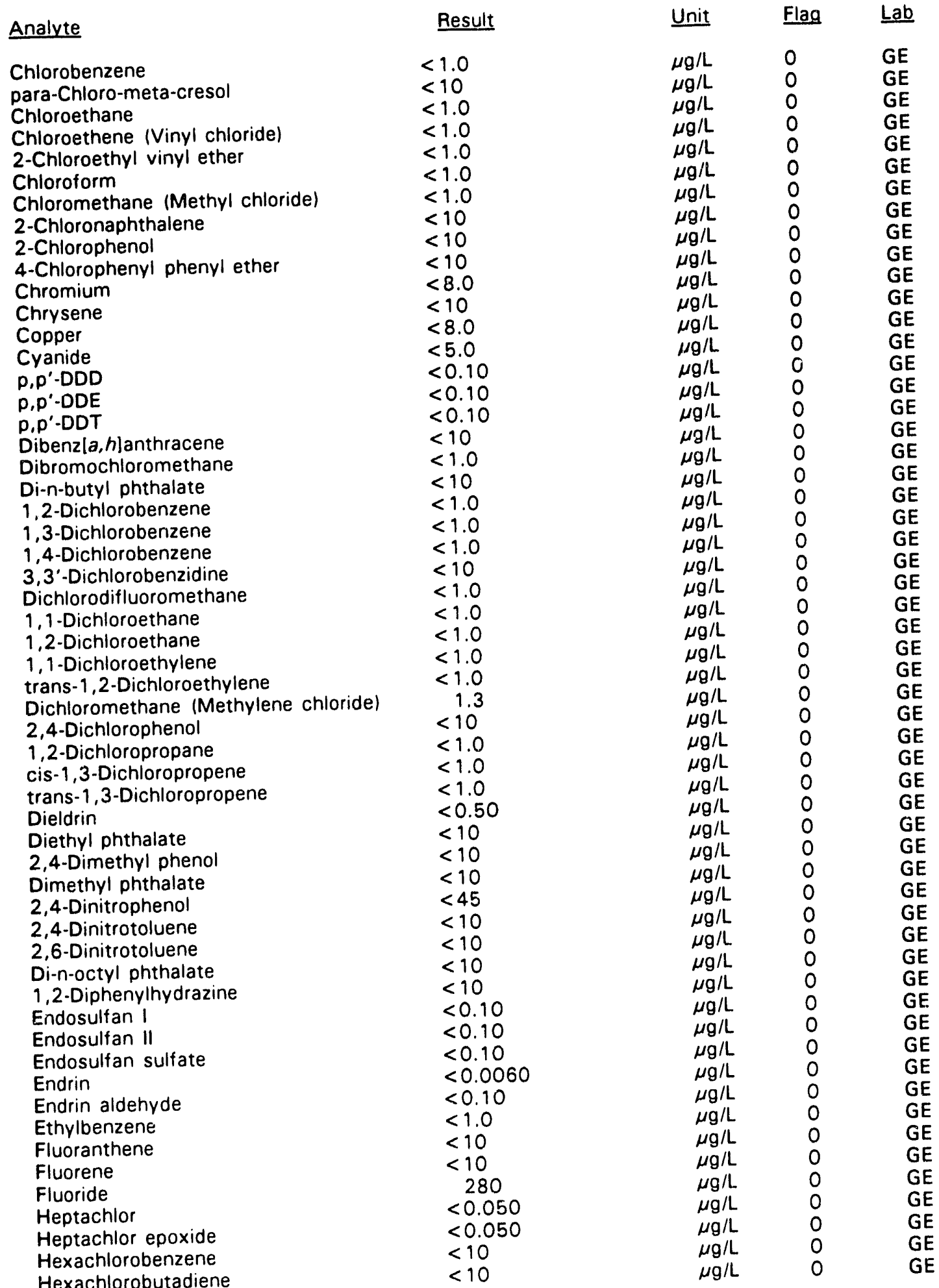

= exceeded holding time. = exceeded primary drinking water standard. 
WELL AMB 1OA collected on 04/29/92, laboratory analyses (cont.)

H D Analyte

Hexachlorocyclopentadiene
Hexachloroethane
Indenol $1,2,3-c$, dpyrene
Iron
Isophorone
Lead
Lindane
Magnesium
Manganese
Mercury
2-Methyl-4,6-dinitrophenol
Naphthalene
Nickel
Nitrate as nitrogen
Nitrobenzene
2-Nitrophenol
4-Nitrophenol
N-Nitrosodimethylamine
N-Nitrosodiphenylamine
N-Nitrosodipropylamine
PCB 1016
PCB 1221
PCB 1232
PCB 1242
PCB 1248
PCB 1254
PCB 1260
Pentachlorophenol
Phenanthrene
Phenol
Phenols
Potassium
Pyrene
Selenium
Silica
Silver
Sodium
Sulfate
$1,1,2,2-$ Tetrachloroethane
Tetrachloroethylene
Thallium
Toluene
Total dissolved solids
Total organic carbon
Total organic halogens
Total phosphates las P)
Toxaphene
1,2,4-Trichlorobenzene
$1,1,1-$ Trichloroethane
1,1,2-Trichloroethane
Trichloroethylene
Trichlorofluoromethane
$2,4,6-$ Trichlorophenol
Zinc
Gross alpha
Nonvolatile beta

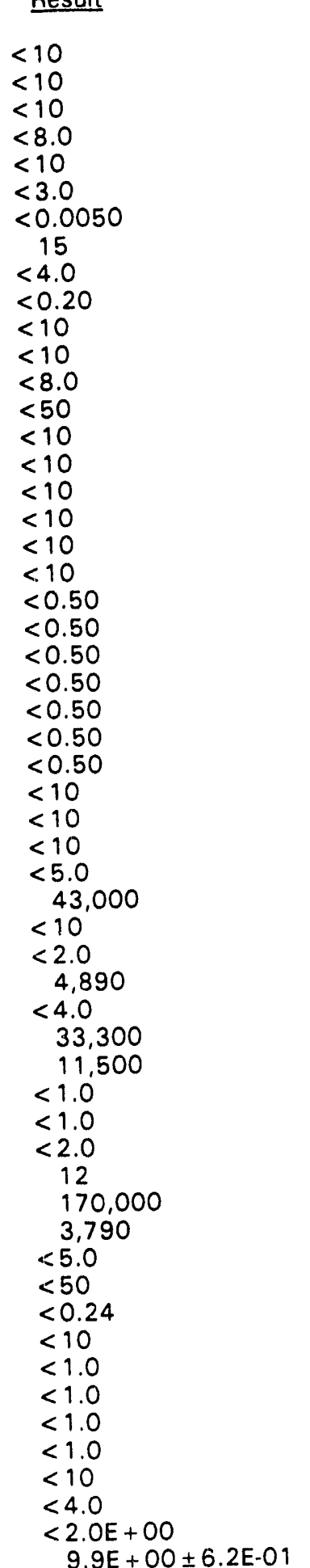

\begin{tabular}{|c|c|c|}
\hline Unit & Flag & $\underline{\text { Lab }}$ \\
\hline$\mu \mathrm{g} / \mathrm{L}$ & 0 & GE \\
\hline$\mu g / L$ & 0 & GE \\
\hline$\mu \mathrm{g} / \mathrm{L}$ & 0 & GE \\
\hline$\mu g / L$ & 0 & GE \\
\hline$\mu \mathrm{g} / \mathrm{L}$ & 0 & GE \\
\hline$\mu g / L$ & 0 & GE \\
\hline $\begin{array}{l}\mu \mathrm{g} / \mathrm{L} \\
\mu \mathrm{g} / \mathrm{L} .\end{array}$ & $\begin{array}{l}0 \\
0\end{array}$ & GE \\
\hline $\begin{array}{l}\mu g / L \\
\mu g / L\end{array}$ & 0 & GE \\
\hline$\mu \mathrm{g} / \mathrm{L}$ & 0 & $\mathrm{GE}$ \\
\hline$\mu g / L$ & 0 & GE \\
\hline$\mu g / L$ & 0 & GE \\
\hline$\mu g / L$ & 0 & GE \\
\hline$\mu g / L$ & 0 & GE \\
\hline$\mu g / L$ & $\begin{array}{l}0 \\
0\end{array}$ & GE \\
\hline $\begin{array}{l}\mu \mathrm{g} / \mathrm{L} \\
\mu \mathrm{g} / \mathrm{L}\end{array}$ & 0 & GE \\
\hline $\begin{array}{l}\mu g / L \\
\mu g / L\end{array}$ & 0 & GE \\
\hline$\mu \mathrm{g} / \mathrm{L}$ & 0 & $\begin{array}{l}\text { GE } \\
\mathrm{GE}\end{array}$ \\
\hline$\mu \mathrm{g} / \mathrm{L}$ & 0 & GE \\
\hline$\mu g / L$ & 0 & GE \\
\hline$\mu g / L$ & 0 & GE \\
\hline$\mu g / L$ & 0 & GE \\
\hline$\mu \mathrm{g} / \mathrm{L}$ & 0 & $\mathrm{GE}$ \\
\hline$\mu g / L$ & 0 & $\mathrm{GE}$ \\
\hline$\mu g / L$ & 0 & $G$ \\
\hline$\mu \mathrm{g} / \mathrm{L}$ & 0 & GE \\
\hline$\mu \mathrm{g} / \mathrm{L}$ & 0 & $\mathrm{GE}$ \\
\hline$\mu g / L$ & 0 & GE \\
\hline$\mu g / L$ & 0 & $\mathbf{G}$ \\
\hline$\mu \mathrm{g} / \mathrm{L}$ & 0 & GE \\
\hline$\mu \mathrm{g} / \mathrm{L}$ & 0 & G \\
\hline $\begin{array}{l}\mu \mathrm{g} / \mathrm{L} \\
\mu \mathrm{g} / \mathrm{L}\end{array}$ & 0 & $\mathrm{G}$ \\
\hline $\begin{array}{l}\mu \mathrm{g} / \mathrm{L} \\
\mu \mathrm{g} / \mathrm{L}\end{array}$ & 0 & 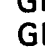 \\
\hline $\begin{array}{l}\mu g / L \\
\mu g / L\end{array}$ & 0 & U \\
\hline$\mu g / L$ & 0 & 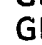 \\
\hline$\mu g / L$ & 0 & $\mathbf{G}$ \\
\hline$\mu \mathrm{g} / \mathrm{L}$ & 0 & $\mathbf{G}$ \\
\hline$\mu g / L$ & 0 & G \\
\hline$\mu \mathrm{g} / \mathrm{L}$ & 0 & G \\
\hline$\mu \mathrm{g} / \mathrm{L}$ & 0 & G \\
\hline$\mu \mathrm{g} / \mathrm{L}$ & 0 & G \\
\hline$\mu g / L$ & 0 & \\
\hline$\mu g / L$ & 0 & \\
\hline$\mu g / L$ & 0 & \\
\hline$\mu \mathrm{g} / \mathrm{L}$ & 0 & \\
\hline$\mu \mathrm{g} / \mathrm{L}$ & 0 & \\
\hline$\mu \mathrm{g} / \mathrm{L}$ & 0 & \\
\hline$\mu \mathrm{g} / \mathrm{L}$ & 0 & \\
\hline$\mu \mathrm{g} / \mathrm{L}$ & 0 & \\
\hline$\mu g / L$ & 0 & \\
\hline$\mu \mathrm{g} / \mathrm{L}$ & 0 & \\
\hline$\mu g / L$ & 0 & \\
\hline pCi/L & 0 & \\
\hline $\mathrm{pCi} / \mathrm{L}$ & 0 & \\
\hline
\end{tabular}

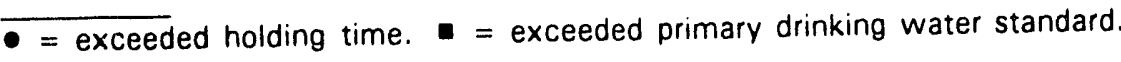


WELL AMB 10A collected on 04/29/92, laboratory analyses (cont.)

$\begin{array}{lllll}\text { H D Analyte } & \underline{\text { Result }} & \underline{\text { Unit }} & \underline{\text { Flag }} & \underline{\text { Lab }} \\ \text { Total alpha-emitting radium } & <1.0 \mathrm{E}+00 & \mathrm{pCi} / \mathrm{L} & 0 & \mathrm{GE} \\ \text { Tritium } & <7.0 \mathrm{E}-01 & \mathrm{pCi} / \mathrm{mL} & 0 & \mathrm{GE} \\ \text { Tritium } & <7.0 \mathrm{E}-01 & \mathrm{pCi} / \mathrm{mL} & 0 & \mathrm{GE}\end{array}$

\section{WELL AMB 10B}

\begin{tabular}{|c|c|c|c|c|c|}
\hline SRS Coord. & Lat/Longitude & Screen Zone Elevation & Top of Casing & Casing & Formation \\
\hline $\begin{array}{l}\text { N103337.3 } \\
\text { E51418.3 }\end{array}$ & $\begin{array}{l}33.3382011^{\circ} \mathrm{N} \\
81.732180^{\circ} \mathrm{W}\end{array}$ & $154.3-149.3 \mathrm{ft} \mathrm{msl}$ & $366.4 \mathrm{ft} \mathrm{msl}$ & 4" PVC & Congaree (IIA) \\
\hline
\end{tabular}

MEASUREMENTS CONDUCTED IN THE FIELD

Sample date: 05/11/92

Depth to water: $143.66 \mathrm{ft}(43.79 \mathrm{~m})$ below TOC

Water elevation: $222.74 \mathrm{ft}(67.89 \mathrm{~m}) \mathrm{msl}$

Sp. conductance: $90 \mu \mathrm{S} / \mathrm{cm}$

Water evacuated before sampling: $288 \mathrm{gal}$

\section{LABORATORY ANALYSES}

H D Analyte

Asbestos

$\mathrm{pH}$

Priority Pollutant Dioxin Screen

Specific conductance

Acenaphthene

Acenaphthylene

Acrolein

Acrylonitrile

Aldrin

Anthracene

Antimony

Antimony

Arsenic

Arsenic

Barium

Benzene

alpha-Benzene hexachloride

beta-Benzene hexachloride

delta-Benzene hexachloride

Benzidine

Benzolajanthracene

Benzolblfluoranthene

Benzolk]fluoranthene

Benzolg, $h, i]$ perylene

Benzolalpyrene

Beryllium

Bis(2-chloroethoxy) methane

Bis(2-chloroethyl) ether

Bis(2-chloroisopropyl) ether

Bis(2-ethyihexyl) phthalate

Bromodichloromethane
Time: $10: 00$

$\mathrm{pH}: 6.4$

Alkalinity: $37 \mathrm{mg} / \mathrm{L}$

Water temperature: $18.9^{\circ} \mathrm{C}$

\begin{tabular}{llll} 
Result & Unit & Flag & Lab \\
\hline 0.15 & $\mathrm{MSL}$ & 0 & $\mathrm{SP}$ \\
7.1 & $\mathrm{pH}$ & 0 & $\mathrm{GE}$ \\
$\mathrm{N}$ & $\mathrm{Y} / \mathrm{N}$ & 0 & $\mathrm{GE}$ \\
90 & $\mu \mathrm{S} / \mathrm{cm}$ & 0 & $\mathrm{GE}$ \\
$<10$ & $\mu \mathrm{g} / \mathrm{L}$ & 0 & $\mathrm{GE}$ \\
$<10$ & $\mu \mathrm{g} / \mathrm{L}$ & 0 & $\mathrm{GE}$ \\
$<20$ & $\mu \mathrm{g} / \mathrm{L}$ & 0 & $\mathrm{GE}$ \\
$<20$ & $\mu \mathrm{g} / \mathrm{L}$ & 0 & $\mathrm{GE}$ \\
$<0.050$ & $\mu \mathrm{g} / \mathrm{L}$ & 0 & $\mathrm{GE}$ \\
$<10$ & $\mu \mathrm{g} / \mathrm{L}$ & 0 & $\mathrm{GE}$ \\
$<2.0$ & $\mu \mathrm{g} / \mathrm{L}$ & 0 & $\mathrm{GE}$ \\
$<2.0$ & $\mu \mathrm{g} / \mathrm{L}$ & 0 & $\mathrm{GE}$ \\
$<2.0$ & $\mu \mathrm{g} / \mathrm{L}$ & 0 & $\mathrm{GE}$ \\
$<2.0$ & $\mu \mathrm{g} / \mathrm{L}$ & 0 & $\mathrm{GE}$ \\
26 & $\mu \mathrm{g} / \mathrm{L}$ & 0 & $\mathrm{GE}$ \\
$<1.0$ & $\mu \mathrm{g} / \mathrm{L}$ & 0 & $\mathrm{GE}$ \\
$<0.050$ & $\mu \mathrm{g} / \mathrm{L}$ & 0 & $\mathrm{GE}$ \\
$<0.050$ & $\mu \mathrm{g} / \mathrm{L}$ & 0 & $\mathrm{GE}$ \\
$<0.050$ & $\mu \mathrm{g} / \mathrm{L}$ & 0 & $\mathrm{GE}$ \\
$<10$ & $\mu \mathrm{g} / \mathrm{L}$ & 0 & $\mathrm{GE}$ \\
$<10$ & $\mu \mathrm{g} / \mathrm{L}$ & 0 & $\mathrm{GE}$ \\
$<10$ & $\mu \mathrm{g} / \mathrm{L}$ & 0 & $\mathrm{GE}$ \\
$<10$ & $\mu \mathrm{g} / \mathrm{L}$ & 0 & $\mathrm{GE}$ \\
$<10$ & $\mu \mathrm{g} / \mathrm{L}$ & 0 & $\mathrm{GE}$ \\
$<10$ & $\mu \mathrm{g} / \mathrm{L}$ & 0 & $\mathrm{GE}$ \\
$<3.0$ & $\mu \mathrm{g} / \mathrm{L}$ & 0 & $\mathrm{GE}$ \\
$<10$ & $\mu \mathrm{g} / \mathrm{L}$ & 0 & $\mathrm{GE}$ \\
$<10$ & $\mu \mathrm{g} / \mathrm{L}$ & 0 & $\mathrm{GE}$ \\
$<10$ & $\mu \mathrm{g} / \mathrm{L}$ & 0 & $\mathrm{GE}$ \\
$<10$ & $\mu \mathrm{g} / \mathrm{L}$ & 0 & $\mathrm{GE}$ \\
$<1.0$ & $\mu \mathrm{g} / \mathrm{L}$ & 0 & $\mathrm{GE}$ \\
$<10$ & & &
\end{tabular}

$\overline{-}=$ exceeded holding time.

- = exceeded primary drinking water standard. 
WELL AMB 10B collected on 05/11/92, laboratory analyses (cont.)

H D Analyte

Bromoform

Bromomethane (Methyl bromide)

4-Bromophenyl phenyl ether

Butylbenzyl phthalate

Cadmium

Calcium

Carbon tetrachloride

Chlordane

Chloride

Chloride

Chlorobenzene

para-Chloro-meta-cresol

Chloroethane

Chloroethene (Vinyl chloride)

2-Chloroethyl vinyl ether

Chloroform

Chloromethane (Methyl chloride)

2-Chloronaphthalene

2-Chlorophenol

4-Chlorophenyl phenyl ether

Chromium

Chrysene

Copper

Cyanide

$p, p^{\prime}-D D D$

$p, p^{\prime}-D D E$

p.p'-DDT

Dibenzla, $h$ lanthracene

Dibromochloromethane

Di-n-butyl phthalate

1,2-Dichlorobenzene

1,3-Dichlorobenzene

1.4-Dichlorobenzene

3,3'-Dichlorobenzidine

Dichlorodifluoromethane

1,1-Dichloroethane

1,2-Dichloroethane

1,9-Dichloroethylene

trans-1,2-Dichloroethylene

Dichloromethane (Methylene chloride)

2,4-Dichlorophenol

1,2-Dichloropropane

cis-1,3-Dichloropropene

trans-1,3-Dichloropropene

Dieldrin

Diethyl phthalate

2,4-Dimethyl phenol

Dimethyl phthalate

2,4-Dinitrophenol

2,4-Dinitrotoluene

2,6-Dinitrotoluene

Di-n-octyl phthalate

1,2-Diphenylhydrazine

Endosulfan I

Endosulfan II

Endosulfan sulfate
Result

$<1.0$

$<1.0$

$<10$

$<10$

$<2.0$

13,200

$<1.0$

$<0.50$

4,060

4,120

$<1.0$

$<10$

$<1.0$

$<1.0$

$<1.0$

$<1.0$

$<1.0$

$<10$

$<10$

$<10$

$<4.0$

$<10$

$<4.0$

$<5.0$

$<0.10$

$<0.10$

$<0.10$

$<10$

$<1.0$

$<10$

$<1.0$

$<1.0$

$<1.0$

$<10$

$<1.0$

$<1.0$

$<1.0$

$<1.0$

$<1.0$

$<1.0$

$<10$

$<1.0$

$<1.0$

$<1.0$

$<0.50$

$<10$

$<10$

$<10$

$<45$

$<10$

$<10$

$=10$

$<10$

$<0.10$

$<0.10$

$<0.10$
Unit Flag Lab

$\mu \mathrm{g} / \mathrm{L}$

$\mu g / L$

$\mu \mathrm{g} / \mathrm{L}$

$\mu g / L$

$\mu g / L$

$\mu g / L$.

$\mu g / L$

$\mu \mathrm{g} / \mathrm{L}$

$\mu g / L$

$\mu \mathrm{g} / \mathrm{L}$

$\mu \mathrm{g} / \mathrm{L}$

$\mu \mathrm{g} / \mathrm{L}$

$\mu \mathrm{g} / \mathrm{L}$

$\mu \mathrm{g} / \mathrm{L}$

$\mu g / L$

$\mu \mathrm{g} / \mathrm{L}$

$\mu g / L$

$\mu g / L$

$\mu \mathrm{g} / \mathrm{L}$

$\mu g / L$

$\mu \mathrm{g} / \mathrm{L}$

$\mu \mathrm{g} / \mathrm{L}$

$\mu g / L$

$\mu \mathrm{g} / \mathrm{L}$

$\mu \mathrm{g} / \mathrm{L}$

$\mu \mathrm{g} / \mathrm{L}$

$\mu \mathrm{g} / \mathrm{L}$

$\mu g / L$

$\mu g / L$

$\mu g /$.

$\mu g / L$

$\mu \mathrm{g} / \mathrm{L}$

$\mu \mathrm{g} / \mathrm{L}$

$\mu \mathrm{g} / \mathrm{L}$

$\mu g / L$

$\mu g / L$

$\mu g / L$

$\mu g / L$

$\mu g / L$

$\mu g / L$

$\mu g / L$

$\mu \mathrm{g} / \mathrm{L}$

$\mu g / L$

$\mu g / L$

$\mu g / L$

$\mu g / L$

$\mu g / L$

$\mu \mathrm{g} / \mathrm{L}$

$\mu g / L$

$\mu g / L$

$\mu g / L$

$\mu g / L$

$\mu g / L$

$\mu g / L$

$\mu g / L$

$\mu g / L$
0 GE

O GE

O GE

O GE

O GE

O GE

O GE

O GE

O GE

O GE

O GE

O GE

O GE

O GE

O GE

O GE

0 GE

O GE

O GE

O GE

O GE

O GE

O GE

O GE

GE

0 GE

O GE

O GE

O GE

O GE

O GE

O GE

O GE

O GE

O GE

O GE

O GE

0 GE

O GE

0 GE

0 GE

O GE

O GE

O GE

0 GE

O GE

0 GE

0 GE

O GE

O GE

O GE

- = exceeded holding time.

- = exceeded primary drinking water standard. 
WELL AMB $10 B$ collected on 05/11/92, laboratory analyses (cont.)

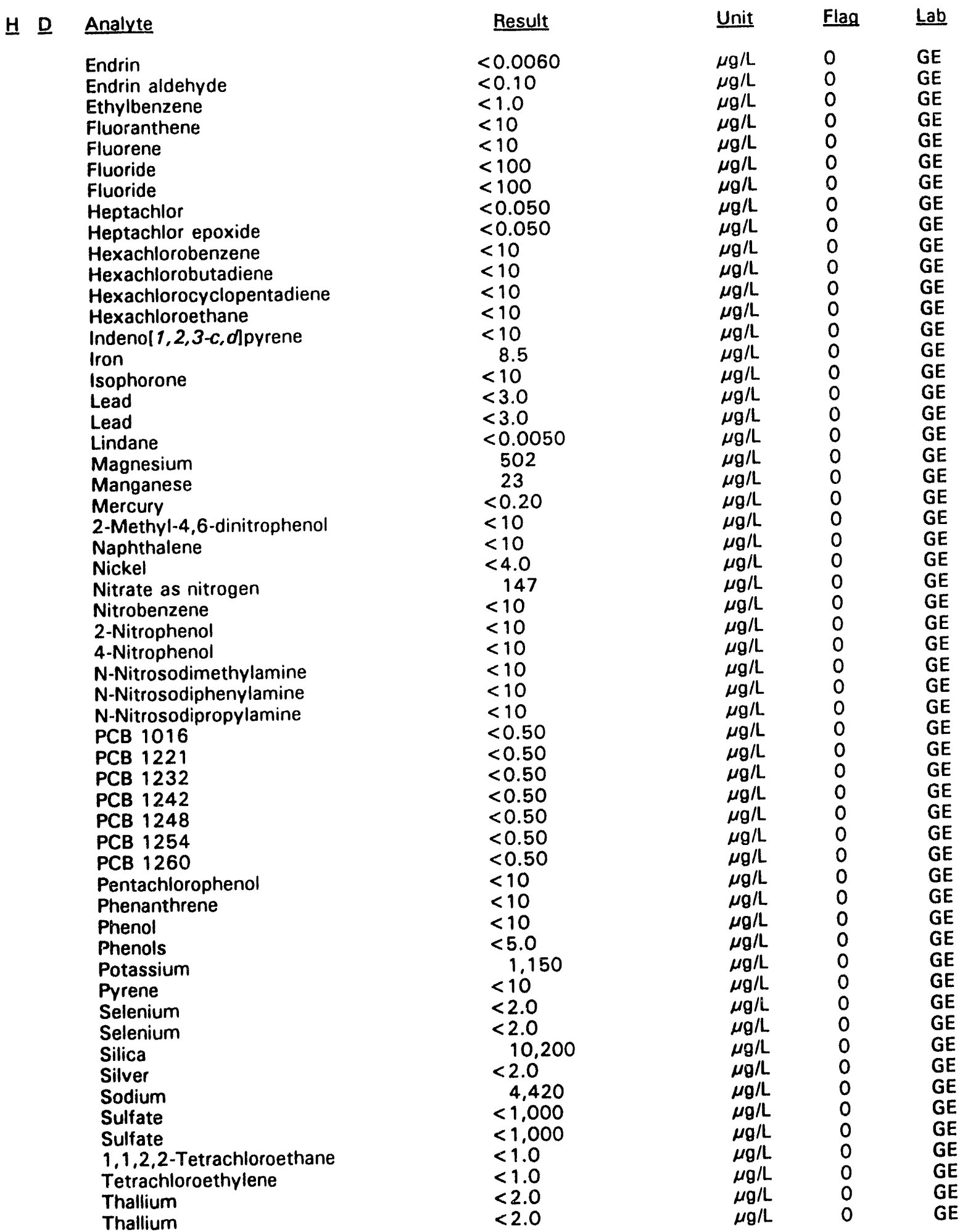

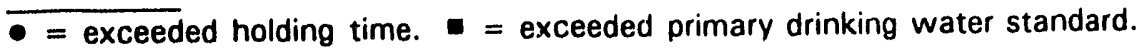


WELL AMB 10B collected on 05/11/92, laboratory analyses (cont.)

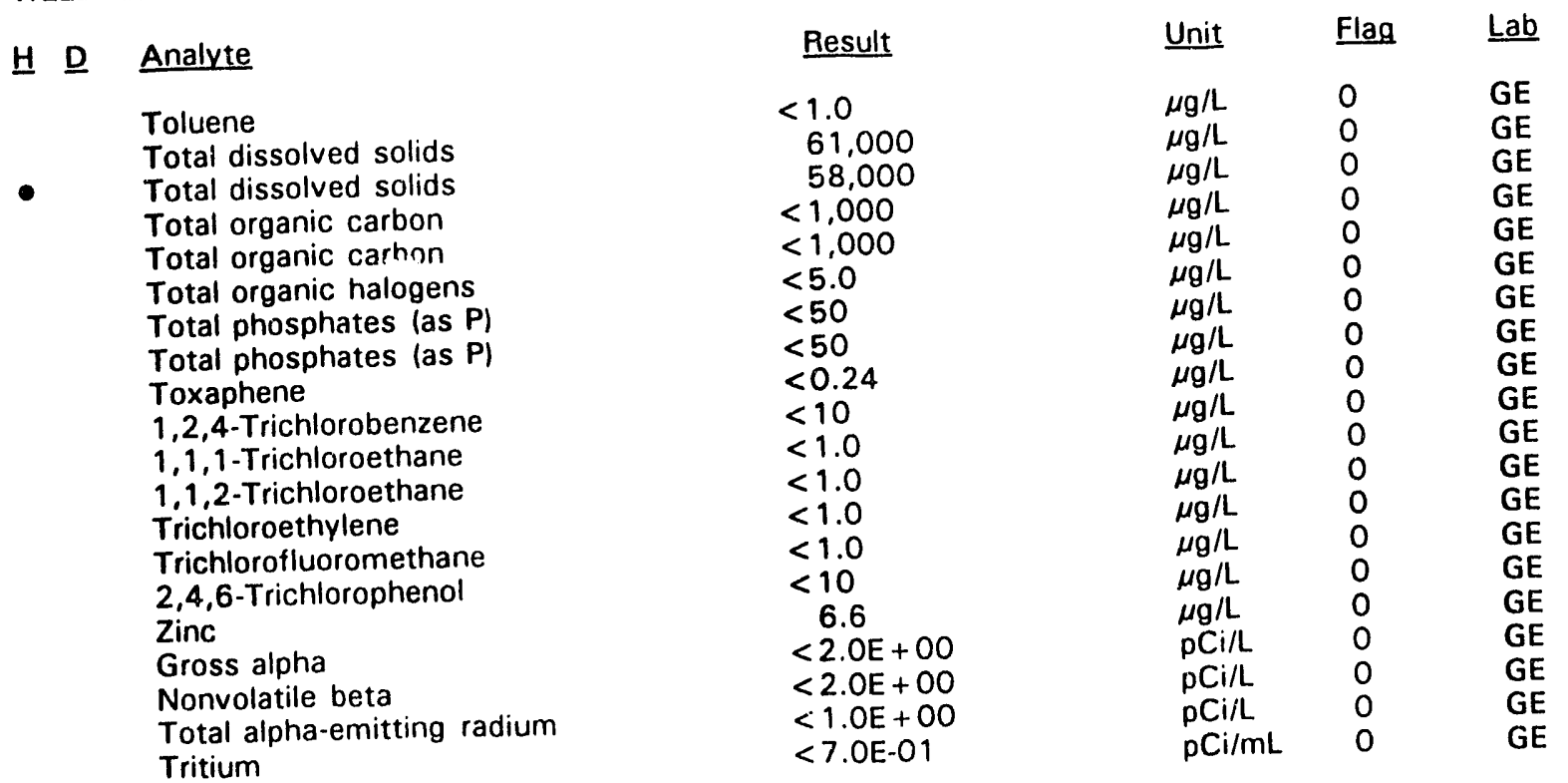

\section{WELL AMB 10D}

\begin{tabular}{|c|c|c|c|c|c|}
\hline SRS Coord. & Lat/Longitude & Screen Zone Elevation & Top of Casing & Casing & Formation \\
\hline 10 & $\begin{array}{l}33.338166^{\circ} \mathrm{N} \\
81.731995^{\circ} \mathrm{W}\end{array}$ & $239.4-219.4 \mathrm{ft} \mathrm{msl}$ & $365.5 \mathrm{ft} \mathrm{msl}$ & 4" PVC & Water Table $\left(1 \mathrm{~B}_{2}\right)$ \\
\hline
\end{tabular}

\section{MEASUREMENTS CONDUCTED IN THE FIELD}

Sample date: 05/11/92

Depth to water: $130.18 \mathrm{ft}(39.68 \mathrm{~m})$ below TOC

Water elevation: $235.32 \mathrm{ft}(71.73 \mathrm{~m}) \mathrm{msl}$

Sp. conductance: $48 \mu \mathrm{S} / \mathrm{cm}$

Water evacuated before sampling: $42 \mathrm{gal}$

\section{LABORATORY ANALYSES}

H D Analyte

- Carbon tetrachloride

- Chloroform

Iron

Lead

Magnesium

Nickel

- Tetrachloroethylene

Total organic carbon

Total organic halogens

Total organic halogens

- 1,1,1-Trichloroethane

- Trichloroethylene

Total alpha-emitting radium
Time: 9:25

$\mathrm{pH}: 5.4$

Alkalinity: $10 \mathrm{mg} / \mathrm{L}$

Water temperature: $17.8^{\circ} \mathrm{C}$

\begin{tabular}{llll} 
Result & $\underline{\text { Unit }}$ & Flag & $\underline{\text { Lab }}$ \\
\cline { 2 - 4 }$<1.0$ & $\mu \mathrm{g} / \mathrm{L}$ & 0 & $\mathrm{GE}$ \\
$<1.0$ & $\mu \mathrm{g} / \mathrm{L}$ & 0 & $\mathrm{GE}$ \\
6.1 & $\mu \mathrm{g} / \mathrm{L}$ & 0 & $\mathrm{GE}$ \\
$<3.0$ & $\mu \mathrm{g} / \mathrm{L}$ & 0 & $\mathrm{GE}$ \\
174 & $\mu \mathrm{g} / \mathrm{L}$ & 0 & $\mathrm{GE}$ \\
$<4.0$ & $\mu \mathrm{g} / \mathrm{L}$ & 0 & $\mathrm{GE}$ \\
$<1.0$ & $\mu \mathrm{g} / \mathrm{L}$ & 0 & $\mathrm{GE}$ \\
$<1.000$ & $\mu \mathrm{g} / \mathrm{L}$ & 0 & $\mathrm{GE}$ \\
7.4 & $\mu \mathrm{g} / \mathrm{L}$ & 0 & $\mathrm{GE}$ \\
5.9 & $\mu \mathrm{g} / \mathrm{L}$ & 0 & $\mathrm{GE}$ \\
$<1.0$ & $\mu \mathrm{g} / \mathrm{L}$ & 0 & $\mathrm{GE}$ \\
$<1.0$ & $\mu \mathrm{g} / \mathrm{L}$ & 0 & $\mathrm{GE}$ \\
$1.4 \mathrm{E}+00 \pm 1.1 \mathrm{E}+00$ & $\mathrm{pCi} / \mathrm{L}$ & 0 & $\mathrm{GE}$
\end{tabular}

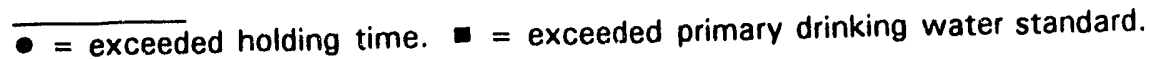


WELL AMB 10DD

$\begin{array}{llllll}\text { SRS Coord. Lat/Longitude } & \text { Screen Zone Elevation } & \text { Top of Casing } & \text { Casing } & \text { Formation } \\ \text { N103278.7 } & \begin{array}{l}33.338133^{\circ} \mathrm{N} \\ \text { E5 } 1456.0\end{array} & 358.6-338.6 \mathrm{ft} \mathrm{msl} & 365.4 \mathrm{ft} \mathrm{msl} & 4^{\text {" PVC }} & \text { Water Table (IIB }{ }_{2} \text { ) }\end{array}$

MEASUREMENTS CONDUCTED IN THE FIELD

Sample date: 05/08/92

Depth to water: $7.93 \mathrm{ft}(2.42 \mathrm{~m})$ below TOC

Water elevation: $357.47 \mathrm{ft}(108.96 \mathrm{~m}) \mathrm{msl}$

Sp. conductance: $135 \mu \mathrm{S} / \mathrm{cm}$

Water evacuated before sampling: $50 \mathrm{gal}$

LABORATORY ANALYSES

H D Analyte

Carbon tetrachloride

Chloroform

Iron

Lead

Magnesium

Nickel

Tetrachloroethylene

Total organic carbon

Total organic halogens

Total organic halogens

1,1,1-Trichloroethane

Trichloroethylene

Total alpha-emitting radium

Total alpha-emitting radium
Time: 16:00

$\mathrm{pH}: 6.4$

Alkalinity: $64 \mathrm{mg} / \mathrm{L}$

Water temperature: $16.6^{\circ} \mathrm{C}$

\section{WELL AMB 11B}

\begin{tabular}{|c|c|c|c|c|c|}
\hline SRS Coord. & Lat/Longitude & Screen Zone Elevation & Top of Casing & Casing & Formation \\
\hline $\begin{array}{l}N 103154.2 \\
\text { E51919.5 }\end{array}$ & $\begin{array}{l}33.338615^{\circ} \mathrm{N} \\
81.730503^{\circ} \mathrm{W}\end{array}$ & $184.5-174.5$ & $364.6 \mathrm{ft} \mathrm{msl}$ & 4" PVC & $e$ (IIA) \\
\hline
\end{tabular}

Result

$<1.0$

$<1.0$

5,840

$<3.0$

443

$<4.0$

$<1.0$

1,140

$<5.0$

$<5.0$

$<1.0$

$<1.0$

$<1.0 E+00$

$<1.0 E+00$

\begin{tabular}{|c|c|}
\hline$\underline{\text { Unit }}$ & Flag \\
\hline$\mu g / L$ & 0 \\
\hline$\mu g / L$ & 0 \\
\hline$\mu g / L$ & 2 \\
\hline$\mu \mathrm{g} / \mathrm{L}$ & 0 \\
\hline$\mu g / L$ & 0 \\
\hline$\mu \mathrm{g} / \mathrm{L}$ & 0 \\
\hline$\mu \mathrm{g} / \mathrm{L}$ & 0 \\
\hline$\mu \mathrm{g} / \mathrm{L}$ & 0 \\
\hline$\mu g / L$ & 0 \\
\hline$\mu g / L$ & 0 \\
\hline$\mu g / L$ & 0 \\
\hline$\mu g / L$ & 0 \\
\hline pCi/L & 0 \\
\hline $\mathrm{pCi} / \mathrm{L}$ & 0 \\
\hline
\end{tabular}

MEASUREMENTS CONDUCTED IN THE FIELD

Sample date: 05/20/92

Depth to water: $141.33 \mathrm{ft}(43.08 \mathrm{~m})$ below TOC

Water elevation: $223.27 \mathrm{ft}(68.05 \mathrm{~m}) \mathrm{msl}$

Sp. conductance: $90 \mu \mathrm{S} / \mathrm{cm}$

Water evacuated before sampling: $160 \mathrm{gal}$

\section{LABORATORY ANALYSES}
H D Analyte
Asbestos
Asbestos
- $\mathrm{pH}$

Result

$$
\begin{array}{r}
<5.0 \\
<5.0 \\
6.4
\end{array}
$$

Time: $10: 30$

pH: 6.1

Alkalinity: $30 \mathrm{mg} / \mathrm{L}$

Water temperature: $18.6^{\circ} \mathrm{C}$

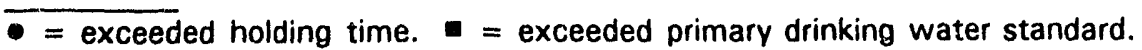

\begin{tabular}{|c|c|}
\hline Unit & Flag \\
\hline MSL & 0 \\
\hline MSL & $\begin{array}{l}0 \\
0\end{array}$ \\
\hline
\end{tabular}


WELL AMB 11 B collected on 05/20/92, laboratory analyses (cont.)

H D Analyte

Priority Pollutant Dioxin Screen

Specific conductance

Acenaphthene

Acenaphthylene

Acrolein

Acrylonitrile

Aldrin

Anthracene

Antimony

Arsenic

Barium

Benzene

alpha-Benzene hexachloride

beta-Benzene hexachloride

delta-Benzene hexachloride

Benzidine

Benzola]anthracene

Benzolblfluoranthene

Benzo[k]fluoranthene

Benzolg,h,ilperylene

Benzola]pyrene

Beryllium

Bis (2-chloroethoxy' methane

Bis (2-chloroethyl) ether

Bis (2-chloroisopropyl) ether

Bis 2-ethylhexyl) phthalate

Bromodichloromethane

Bromoform

Bromomethane (Methyl bromide)

4-Bromophenyl phenyl ether

Butylbenzyl phthalate

Cadmium

Calcium

Carbon tetrachloride

Chlordane

Chloride

Chlorobenzene

para-Chloro-meta-cresol

Chloroethane

Chloroethene (Vinyl chloide)

2-Chloroethyl vinyl ether

Chloroform

Chloromethane (Methyl chioride)

2-Chloronaphthalene

2-Chlorophenol

4-Chlorophenyl phenyl ether

Chromium

Chrysene

Copper

Cyanide

$p, p^{\prime}-D D D$

$p, p^{\prime}-D D E$

$p, p^{\prime} \cdot D D T$

Dibenzla, hlanthracene

Dibromochloromethane

Di-n-butyl phthalate

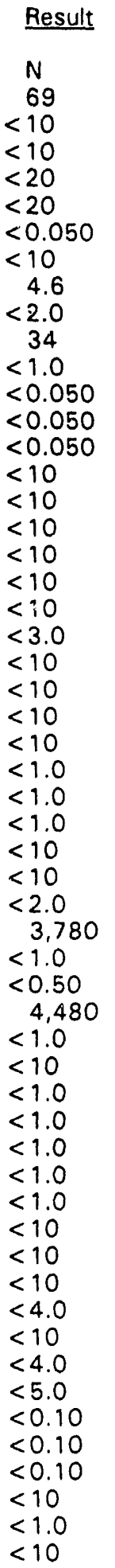

\begin{tabular}{|c|c|c|}
\hline Unit & Flag & $\underline{L a b}$ \\
\hline$Y / N$ & 0 & GE \\
\hline$\mu \mathrm{S} / \mathrm{cm}$ & 0 & GE \\
\hline$\mu \mathrm{g} / \mathrm{L}$ & 0 & GE \\
\hline$\mu g / L$ & 0 & GE \\
\hline$\mu g / L$ & 0 & GE \\
\hline$\mu \mathrm{g} / \mathrm{L}$ & 0 & GE \\
\hline$\mu g / L$ & 0 & $\mathrm{GE}$ \\
\hline$\mu g / L$ & 0 & GE \\
\hline$\mu g / L$ & 1 & $\mathrm{GE}$ \\
\hline$\mu g / L$ & 0 & GE \\
\hline$\mu g / L$ & 0 & GE \\
\hline$\mu g / L$ & 0 & GE \\
\hline$\mu g / L$ & 0 & GE \\
\hline$\mu g / L$ & 0 & GE \\
\hline$\mu g / L$ & 0 & $\mathrm{GE}$ \\
\hline$\mu \mathrm{g} / \mathrm{L}$ & 0 & GE \\
\hline$\mu \mathrm{g} / \mathrm{L}$ & 0 & GE \\
\hline$\mu \mathrm{g} / \mathrm{L}$ & 0 & GE \\
\hline$\mu \mathrm{g} / \mathrm{L}$ & 0 & GE \\
\hline$\mu \mathrm{g} / \mathrm{L}$ & 0 & GE \\
\hline$\mu \mathrm{g} / \mathrm{L}$ & 0 & GE \\
\hline$\mu \mathrm{g} / \mathrm{L}$ & 0 & GE \\
\hline$\mu \mathrm{g} / \mathrm{L}$ & 0 & GE \\
\hline$\mu \mathrm{g} / \mathrm{L}$ & 0 & $\mathrm{GE}$ \\
\hline$\mu \mathrm{g} / \mathrm{L}$ & 0 & GE \\
\hline$\mu \mathrm{g} / \mathrm{L}$ & 0 & GE \\
\hline$\mu \mathrm{g} / \mathrm{L}$ & 0 & GE \\
\hline$\mu g / L$ & 0 & GE \\
\hline$\mu \mathrm{g} / \mathrm{L}$ & 0 & $\mathrm{GE}$ \\
\hline$\mu \mathrm{g} / \mathrm{L}$ & 0 & GE \\
\hline$\mu \mathrm{g} / \mathrm{L}$ & 0 & GE \\
\hline$\mu \mathrm{g} / \mathrm{L}$ & 0 & GE \\
\hline$\mu g / L$ & 0 & GE \\
\hline$\mu \mathrm{g} / \mathrm{L}$ & 0 & $\mathrm{GE}$ \\
\hline$\mu \mathrm{g} / \mathrm{L}$ & 0 & GE \\
\hline$\mu \mathrm{g} / \mathrm{L}$ & 0 & GE \\
\hline$\mu g / L$ & 0 & GE \\
\hline$\mu \mathrm{g} / \mathrm{L}$ & 0 & GE \\
\hline$\mu \mathrm{g} / \mathrm{L}$ & 0 & GE \\
\hline$\mu \mathrm{g} / \mathrm{L}$ & 0 & GE \\
\hline$\mu \mathrm{g} / \mathrm{L}$ & 0 & GE \\
\hline$\mu \mathrm{g} / \mathrm{L}$ & 0 & GE \\
\hline$\mu g / L$ & 0 & GE \\
\hline$\mu g / L$ & 0 & GE \\
\hline$\mu g / L$ & 0 & $\mathrm{GE}$ \\
\hline$\mu g / L$ & 0 & iE \\
\hline$\mu g / L$ & 0 & GE \\
\hline$\mu \mathrm{g} / \mathrm{L}$ & 0 & GE \\
\hline$\mu \mathrm{g} / \mathrm{L}$ & 0 & GE \\
\hline$\mu \mathrm{g} / \mathrm{L}$ & 0 & GE \\
\hline$\mu \mathrm{g} / \mathrm{L}$ & 0 & \\
\hline$\mu \mathrm{g} / \mathrm{L}$ & 0 & E \\
\hline$\mu \mathrm{g} / \mathrm{L}$ & 0 & GE \\
\hline$\mu \mathrm{g} / \mathrm{L}$ & 0 & G \\
\hline$\mu g / L$ & 0 & $G$ \\
\hline$\mu g / L$ & 0 & \\
\hline
\end{tabular}

$\overline{-} \overline{\text { exceeded holding time. }} \mathbf{E}=$ exceeded primary drinking water standard. 
WELL AMB 11 B collected on 05/20/92, laboratory analyses (cont.)

H D Analyte

1,2-Dichlorobenzene

1,3-Dichlorobenzene

1,4 -Dichlorobenzene

3,3'-Dichlorobenzidine

Dichlorodifluoromethane

1,1-Dichloroethane

1,2-Dichloroethane

1,1-Dichloroethylene

trans-1,2-Dichloroethylene

Dichloromethane (Methylene chloride)

2,4-Dichlorophenol

1,2-Dichloropropane

cis-1,3-Dichloropropene

trans-1,3-Dichloropropene

Dieldrin

Diethyl phthalate

2,4-Dimethyl phenol

Dimethyl phthalate

2,4-Dinitrophenol

2,4-Dinitrotoluene

2,6-Dinitrotoluene

Di-n-octyl phthalate

1,2-Diphenylhydrazine

Endosulfan I

Endosulfan II

Endosulfan sulfate

Endrin

Endrin aldehyde

Ethylbenzene

Fluoranthene

Fluorene

Fluoride

Heptachlor

Heptachlor epoxide

Hexachlorobenzene

Hexachluroisutadiene

Hexachlororvclopentadiene

Hexachlorounane

Indenol 1,2,3-c. cipyrene

Iron

Isophorone

Lead

Lindane

Magnesium

Manganese

Mercury

Mercury

2-Methyl-4,6-dinitrophenol

Naphthalene

Nickel

Nitrate as nitrogen

Nitrobenzene

2-Nitrophenol

4-Nitrophenol

$\mathrm{N}$-Nitrosodimethylamine

N-Nitrosodiphenylamine
Result

$<1.0$

$<1.0$

$<1.0$

$<10$

$<1.0$

$<1.0$

$<1.0$

$<1.0$

$<1.0$

1.5

$<10$

$<1.0$

$<1.0$

$<1.0$

$<0.50$

$<10$

$<10$

$<10$

$<45$

$<10$

$<10$

$<10$

$<10$

$<0.10$

$<0.10$

$<0.10$

$<0.0060$

$<0.10$

$<1.0$

$<10$

$<10$

$<100$

$<0.050$

$<0.050$

$<10$

$<10$

$<10$

$<10$

$<10$

$<4.0$

$<10$

$<3.0$

$<0.0050$

354

18

$<0.20$

$<0.20$

$<10$

$<10$

$<4.0$

630

$<10$

$<10$

$<10$

$<10$

$<10$ $\underline{\text { Unit }} \quad \underline{\text { Flag }} \quad \underline{\text { Lab }}$

$\mu \mathrm{g} / \mathrm{L} \quad 0 \quad \mathrm{GE}$

$\mu \mathrm{g} / \mathrm{L} \quad \mathrm{O} \quad \mathrm{GE}$

$\mu \mathrm{g} / \mathrm{L} \quad \mathrm{O} \quad \mathrm{GE}$

$\mu g / L \quad O \quad G E$

$\mu g / L \quad 0 \quad$ GE

$\mu g / L \quad O \quad$ GE

$\mu \mathrm{g} / \mathrm{L} \quad \mathrm{O} \quad \mathrm{GE}$

$\mu \mathrm{g} / \mathrm{L} \quad 0 \quad \mathrm{GE}$

$\mu g / L \quad 0 \quad G E$

$\mu g / L \quad O \quad G E$

$\mu g / L \quad 0 \quad G E$

$\mu \mathrm{g} / \mathrm{L} \quad \mathrm{O} \quad \mathrm{GE}$

$\mu \mathrm{g} / \mathrm{L} \quad \mathrm{O} \quad \mathrm{GE}$

$\mu \mathrm{g} / \mathrm{L} \quad \mathrm{O} \quad \mathrm{GE}$

$\mu g / L \quad 0 \quad G E$

$\mu \mathrm{g} / \mathrm{L} \quad \mathrm{O} \quad \mathrm{GE}$

$\mu g / L \quad 0 \quad G E$

$\mu \mathrm{g} / \mathrm{L} \quad \mathrm{O} \quad \mathrm{GE}$

$\mu g / L \quad 0 \quad G E$

$\mu \mathrm{g} / \mathrm{L} \quad \mathrm{O} \quad \mathrm{GE}$

$\mu \mathrm{g} / \mathrm{L} \quad \mathrm{O} \quad \mathrm{GE}$

$\mu \mathrm{g} / \mathrm{L} \quad \mathrm{O} \quad \mathrm{GE}$

$\mu \mathrm{g} / \mathrm{L} \quad 0 \quad \mathrm{GE}$

$\mu \mathrm{g} / \mathrm{L} \quad 0 \quad \mathrm{GE}$

$\mu g / L \quad 0 \quad G E$

$\mu \mathrm{g} / \mathrm{L} \quad 0 \quad \mathrm{GE}$

$\mu g / L \quad 0 \quad G E$

$\mu \mathrm{g} / \mathrm{L} \quad \mathrm{O} \quad \mathrm{GE}$

$\mu g / L \quad 0 \quad G E$

$\mu \mathrm{g} / \mathrm{L} \quad \mathrm{O} \quad \mathrm{GE}$

$\mu g / L \quad 0 \quad$ GE

$\mu \mathrm{g} / \mathrm{L} \quad \mathrm{O} \quad \mathrm{GE}$

$\mu g / L \quad 0 \quad$ GE

$\mu \mathrm{g} / \mathrm{L} \quad \mathrm{O} \quad \mathrm{GE}$

$\mu \mathrm{g} / \mathrm{L} \quad \mathrm{O} \quad \mathrm{GE}$

$\mu \mathrm{g} / \mathrm{L} \quad \mathrm{O} \quad \mathrm{GE}$

$\mu \mathrm{g} / \mathrm{L} \quad 0 \quad \mathrm{GE}$

$\mu \mathrm{g} / \mathrm{L} \quad 0 \quad \mathrm{GE}$

$\mu g / L \quad 0 \quad G E$

$\mu \mathrm{g} / \mathrm{L} \quad \mathrm{O} \quad \mathrm{GE}$

$\mu \mathrm{g} / \mathrm{L} \quad 0 \quad \mathrm{GE}$

$\mu g / L \quad O \quad G E$

$\mu \mathrm{g} / \mathrm{L} \quad 0 \quad \mathrm{GE}$

$\mu \mathrm{g} / \mathrm{L} \quad \mathrm{O} \quad \mathrm{GE}$

$\mu \mathrm{g} / \mathrm{L} \quad 0 \quad \mathrm{GE}$

$\mu \mathrm{g} / \mathrm{L} \quad 0 \quad \mathrm{GE}$

$\mu \mathrm{g} / \mathrm{L} \quad \mathrm{O} \quad \mathrm{GE}$

$\mu \mathrm{g} / \mathrm{L} \quad 0 \quad \mathrm{GE}$

$\mu \mathrm{g} / \mathrm{L} \quad \mathrm{O} \quad \mathrm{GE}$

$\mu \mathrm{g} / \mathrm{L} . \quad \mathrm{G} \quad \mathrm{GE}$

$\mu \mathrm{g} / \mathrm{L} \quad \mathrm{O} \quad \mathrm{GE}$

$\mu \mathrm{g} / \mathrm{L} \quad 0 \quad \mathrm{GE}$

$\mu \mathrm{g} / \mathrm{L} \quad \mathrm{O} \quad \mathrm{GE}$

$\mu \mathrm{g} / \mathrm{L} \quad 0 \quad \mathrm{GE}$

$\mu \mathrm{g} / \mathrm{L} \quad 0 \quad \mathrm{GE}$

$\mu \mathrm{g} / \mathrm{L} \quad \mathrm{O} \quad \mathrm{GE}$

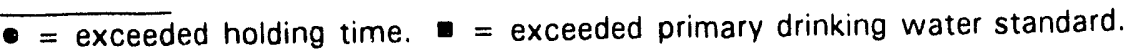


WELL AMB 11 B collected on 05/20/92, laboratory analyses (cont.)

H $\underline{\text { Analyte }}$

N-Nitrosodipropylamine

PCB 1016

PCB 1221

PCB 1232

РCB 1242

PCB 1248

PCB 1254

PCB 1260

Pentachlorophenol

Phenanthrene

Phenol

Phenols

Potassium

Pyrene

Selenium

Silica

Silver

Sodium

Sulfate

1,1,2,2-Tetrachloroethane

Tetrachloroethylene

Thallium

Toluene

- Total dissolved solids

Total organic carbon

Total organic halogens

Total phosphates (as P)

Toxaphene

1,2,4-Trichlorobenzene

1,1,1-Trichloroethane

1,1,2-Trichloroethane

Trichloroethylene

Trichlorofluoromethane

2,4,6-Trichlorophenol

Zinc

Gross alpha

Nonvolatile beta

Total alpha-emitting radium

Tritium
Result

$<10$

$<0.50$

$<0.50$

$<0.50$

$<0.50$

$<0.50$

$<0.50$

$<0.50$

$<10$

$<10$

$<10$

$<5.0$

1,370

$<10$

$<2.0$

9,870

$<2.0$

10,400

1,680

$<1.0$

$<1.0$

$<2.0$

$<1.0$

66,000

$<1,000$

14

80

$<0.24$

$<10$

$<1.0$

$<1.0$

$<1.0$

8.4

$<10$

5.7

$3.0 E+00 \pm 5.1 E-01$

$3.9 \mathrm{E}+00 \pm 5.5 \mathrm{E}-01$

$3.4 \mathrm{E}+00 \pm 1.4 \mathrm{E}+00$

$<7.0$-01

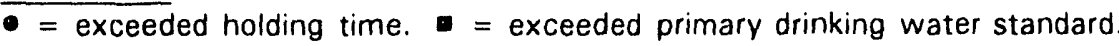


WELL AMB 11D

\begin{tabular}{|c|c|c|c|c|c|}
\hline SRS Coord. & Lat/Lonqitude & Screen Zone Elevation & Top of Casing & Casing & Formation \\
\hline & $\begin{array}{l}33.338588^{\circ} \mathrm{N} \\
81.730426 \circ \mathrm{W}\end{array}$ & 1 & 36 & 4" PVC & \\
\hline
\end{tabular}

MEASUREMENTS CONDUCTED IN THE FIELD

Sample date: 05/20/92

Depth to water: $127.62 \mathrm{ft}(38.90 \mathrm{~m})$ below TOC

Water elevation: $236.38 \mathrm{ft}(72.05 \mathrm{~m}) \mathrm{msl}$

Sp. conductance: $104 \mu \mathrm{S} / \mathrm{cm}$

Water evacuated before sampling: 62 gal

LABORATORY ANALYSES

H D Analyte

Carbon tetrachloride
Chloroform
Iron
Lead
Magnesium
Nickel
Tetrachloroethylene
Total organic carbon
Total organic halogens
Total organic halogens
$1,1,1$-Trichloroethane
Trichloroethylene
Toial alpha-emitting radium

Result

$<1.0$

$<1.0$

$<4.0$

$<3.0$

59

$<4.0$

$<1.0$

$<1,000$

$<5.0$

$<5.0$

$<1.0$

$<1.0$

$<1.0 E+00$
Time: $9: 30$

$\mathrm{pH}: 6.7$

Alkalinity: $33 \mathrm{mg} / \mathrm{L}$

Water temperature: $17.3^{\circ} \mathrm{C}$

WELL AMB 12D

\begin{tabular}{|c|c|c|c|c|c|}
\hline SRS Coord. & Lat/Longitude & Screen Zone Elevation & Top of Casing & Casing & Formation \\
\hline & 7 & $239.4-219.4 \mathrm{ft} \mathrm{msl}$ & $36 s$ & 4" PVC & \\
\hline
\end{tabular}

\begin{tabular}{|c|c|}
\hline Unit & Flag \\
\hline$\mu \mathrm{g} / \mathrm{L}$ & 0 \\
\hline$\mu \mathrm{g} / \mathrm{L}$ & 0 \\
\hline$\mu g / L$ & 0 \\
\hline$\mu g / L$ & 0 \\
\hline$\mu \mathrm{g} / \mathrm{L}$ & 0 \\
\hline$\mu g / L$ & 0 \\
\hline$\mu g / L$ & 0 \\
\hline$\mu g / L$ & 0 \\
\hline$\mu g / L$ & 0 \\
\hline$\mu \mathrm{g} / \mathrm{L}$ & 0 \\
\hline$\mu g / L$ & 0 \\
\hline$\mu \mathrm{g} / \mathrm{L}$ & 0 \\
\hline
\end{tabular}

Lab
GE
GE
GE
GE
GE
GE
GE
GE
GE
GE
GE
GE
GE $\begin{array}{ll}\mathrm{E} 51901.6 & 81.731422^{\circ} \mathrm{W}\end{array}$

Time: $12: 30$

$\mathrm{pH}: 5.5$

Alkalinity: $9 \mathrm{mg} / \mathrm{L}$

Water temperature: $20.9^{\circ} \mathrm{C}$

Depth to water: $134.73 \mathrm{ft}(41.07 \mathrm{~m})$ below TOC

Water elevation: $235.07 \mathrm{ft}\left(7^{\circ} .65 \mathrm{~m}\right) \mathrm{msl}$

Sp. conductance: $34 \mu \mathrm{S} / \mathrm{cm}$

Water evacuated before sampling: $61 \mathrm{gal}$

\section{LABORATORY ANALYSES}

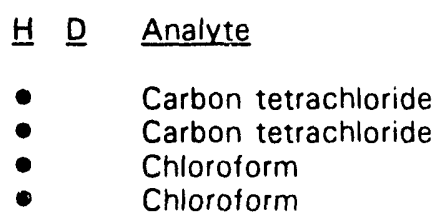

H D Analyte

Carbon tetrachioride

Chloroform

Chloroform

$$
\begin{aligned}
& <1.0 \\
& <1.0 \\
& <1.0 \\
& <1.0
\end{aligned}
$$

$\underline{\text { Result }}$
Unit

$\mu \mathrm{g} / \mathrm{L}$

$\mu \mathrm{g} / \mathrm{L}$

$\mu g / L$

$\mu g / L$
Flag Lab

$\begin{array}{ll}0 & \text { GE } \\ 0 & \text { GE } \\ 0 & \text { GE } \\ 0 & \text { GE }\end{array}$

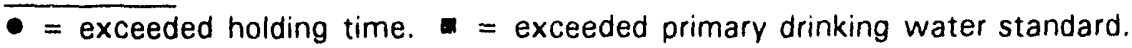


WELL AMB 12D collected on 05/12/92, laboratory analyses (cont.)

H D Analyte

Iron

Lead

Magnesium

Nickel

- Tetrachloroethylene

Tetrachloroethylene

Total organic carbon

Total organic halogens

Total organic halogens

- 1,1,1-Trichloroethane

$1,1,1$-Trichloroethane

Trichloroethylene

Trichloroethylene

Total alpha-emitting radium
Result

18

$<3.0$

306

$<4.0$

$<1.0$

$<1.0$

$<1,000$

13

12

$<1.0$

$<1.0$

$<1.0$

$<1.0$

$<1.0 \mathrm{OE}+00$ $\underline{\text { Unit }} \quad \underline{\text { Flag }} \quad \underline{\text { Lab }}$

$\mu g / L \quad 0 \quad$ GE

$\mu g / L \quad 0 \quad G E$

$\mu \mathrm{g} / \mathrm{L} \quad \mathrm{O} \quad \mathrm{GE}$

$\mu g / L \quad 0 \quad$ GE

$\mu g / L \quad O \quad G E$

$\mu \mathrm{g} / \mathrm{L} \quad \mathrm{O}$

$\mu g / L \quad O \quad G E$

$\mu g / L \quad 0$

$\mu g / L \quad 0 \quad$ GE

$\mu \mathrm{g} / \mathrm{L} \quad \mathrm{O} \quad \mathrm{GE}$

$\mu g / L \quad O \quad G E$

$\mu g / L \quad O \quad G E$

$\mu g / L \quad 0$

$\mathrm{pCi} / \mathrm{L} \quad \mathrm{G} \quad \mathrm{GE}$

\section{WELL AMB 13AR}

\begin{tabular}{|c|c|c|c|c|c|}
\hline SRS Coord. & Lat/Longitude & Screen Zone Elevation & Top of Casing & Casing & Formation \\
\hline $\begin{array}{l}\text { N103082.0 } \\
\text { E51396.0 }\end{array}$ & $\begin{array}{l}33.337600^{\circ} \mathrm{N} \\
81.731742^{\circ} \mathrm{W}\end{array}$ & 110 & 3 & PVC & d) \\
\hline
\end{tabular}

\section{MEASUREMENTS CONDUCTED IN THE FIELD}

Sample date: 05/08/92

Depth to water: $146.47 \mathrm{ft}(44.64 \mathrm{~m})$ below TOC

Water elevation: $218.63 \mathrm{ft}(66.64 \mathrm{~m}) \mathrm{msl}$

Sp. conductance: $106 \mu \mathrm{S} / \mathrm{cm}$

Vifater evacuated before sampling: $386 \mathrm{gal}$

\section{LABORATORY ANALYSES}

H D Analyte

Asbestos

$\mathrm{pH}$

Priority Pollutant Dioxin Screen

Specific conductance

Acenaphthene

Acenaphthylene

Acrolein

Acrylonitrile

Aldrin

Anthracene

Antimony

Arsenic

Barium

Barium

Benzene

alpha-Benzene hexachloride

beta-Benzene hexachloride

delta-Benzene hexachloride

Benzidine

Benzola]anthracene
Time: 15:15

$\mathrm{pH}: 7.3$

Alkalinity: $40 \mathrm{mg} / \mathrm{L}$

Water temperature: $18.7^{\circ} \mathrm{C}$

\begin{tabular}{llll} 
Result & Unit & Flag & Lab \\
\hline$<5.0$ & $\mathrm{MSL}$ & 0 & $\mathrm{SP}$ \\
7.7 & $\mathrm{pH}$ & 0 & $\mathrm{GE}$ \\
$N$ & $\mathrm{Y} / \mathrm{N}$ & 0 & $\mathrm{GE}$ \\
105 & $\mu \mathrm{S} / \mathrm{cm}$ & 0 & $\mathrm{GE}$ \\
$<10$ & $\mu \mathrm{g} / \mathrm{L}$ & 0 & $\mathrm{GE}$ \\
$<10$ & $\mu \mathrm{g} / \mathrm{L}$ & 0 & $\mathrm{GE}$ \\
$<20$ & $\mu \mathrm{g} / \mathrm{L}$ & 0 & $\mathrm{GE}$ \\
$<20$ & $\mu \mathrm{g} / \mathrm{L}$ & 0 & $\mathrm{GE}$ \\
$<0.050$ & $\mu \mathrm{g} / \mathrm{L}$ & 0 & $\mathrm{GE}$ \\
$<10$ & $\mu \mathrm{g} / \mathrm{L}$ & 0 & $\mathrm{GE}$ \\
$<2.0$ & $\mu \mathrm{g} / \mathrm{L}$ & 0 & $\mathrm{GE}$ \\
$<2.0$ & $\mu \mathrm{g} / \mathrm{L}$ & 0 & $\mathrm{GE}$ \\
41 & $\mu \mathrm{g} / \mathrm{L}$ & 0 & $\mathrm{GE}$ \\
42 & $\mu \mathrm{g} / \mathrm{L}$ & 0 & $\mathrm{GE}$ \\
$<1.0$ & $\mu \mathrm{g} / \mathrm{L}$ & 0 & $\mathrm{GE}$ \\
$<0.050$ & $\mu \mathrm{g} / \mathrm{L}$ & 0 & $\mathrm{GE}$ \\
$<0.050$ & $\mu \mathrm{g} / \mathrm{L}$ & 0 & $\mathrm{GE}$ \\
$<0.050$ & $\mu \mathrm{g} / \mathrm{L}$ & 0 & $\mathrm{GE}$ \\
$<10$ & $\mu \mathrm{g} / \mathrm{L}$ & 0 & $\mathrm{GE}$ \\
$<10$ & $\mu \mathrm{g} / \mathrm{L}$ & 0 & $\mathrm{GE}$
\end{tabular}

- exceeded holding time. = exceeded primary drinking water standard. 
WELL AMB 13AR collected on 05/08/92, laboratory analyses (cont.)

H D Analyte

Benzo[b]fluoranthene

Benzolk]fluoranthene

Benzolg,h,i]perylene

Benzolalpyrene

Beryllium

Beryllium

Bis (2-chloroethoxy) methane

Bis(2-chloroethyl) ether

Bis(2-chloroisopropyl) ether

Bis(2-ethyihexyl) phthalate

Bromodichloromethane

Bromotorm

Bromomethane (Methyl bromide)

4-Bromophenyl phenyl ether

Butylbenzyl phthalate

Cadmium

Cadmium

Calcium

Calcium

Carbon tetrachloride

Chlordane

Chloride

Chlorobenzene

para-Chloro-meta-cresol

Chloroethane

Chloroethene (Vinyl chloride)

2-Chloroethyl vinyl ether

Chloroform

Chloromethane (Methyl chloride)

2-Chloronaphthalene

2-Chlorophenol

4-Chlorophenyl phenyl ether

Chromium

Chromium

Chrysene

Copper

Copper

- Cyanide

$p, p^{\prime}-D D D$

$p, p^{\prime}-D D E$

$p, p^{\prime}-$ DDT

Dibenz $[a, h]$ anthracene

Dibromochloromethane

Di-n-butyl phthalate

1,2-Dichlorobenzene

1,3-Dichlorobenzene

1,4-Dichlorobenzene

3, 3'-Dichlorobenzidine

Dichlorodifluoromethane

1.1-Dichloroethane

1,2-Dichloroethane

1,1-Dichloroethylene

trans-1,2-Dichloroethylene

Dichloromethane (Methylene chloride)

2,4-Dichlorophenol

1,2-Dichloropropane
Result

$<10$

$<10$

$<10$

$<10$

$<3.0$

$<3.0$

$<10$

$<10$

$<10$

$<10$

$<1.0$

$<1.0$

$<1.0$

$<10$

$<10$

$<2.0$

$<2.0$

12,000

12,100

$<1.0$

$<0.50$

2.260

$<1.0$

$<10$

$<1.0$

$<1.0$

$<1.0$

$<1.0$

$<1.0$

$<10$

$<10$

$<10$

$<4.0$

$<4.0$

$<10$

5.3

5.3

$<5.0$

$<0.10$

$<0.10$

$<0.10$

$<10$

$<1.0$

$<10$

$<1.0$

$<1.0$

$<1.0$

$<10$

$<1.0$

$<1.0$

$<1.0$

$<1.0$

$<1.0$

1.3

$<10$

$<1.0$

\begin{tabular}{|c|c|c|}
\hline Unit & Flag & $\underline{L a b}$ \\
\hline$\mu g / L$ & 0 & GE \\
\hline$\mu \mathrm{g} / \mathrm{L}$ & 0 & GE \\
\hline$\mu \mathrm{g} / \mathrm{L}$ & 0 & GE \\
\hline$\mu g / L$ & 0 & GE \\
\hline$\mu g / L$ & 0 & GE \\
\hline$\mu \mathrm{g} / \mathrm{L}$ & 0 & $\mathrm{GE}$ \\
\hline$\mu g / L$ & 0 & GE \\
\hline$\mu g / L$ & 0 & GE \\
\hline$\mu g / L$ & 0 & GE \\
\hline$\mu \mathrm{g} / \mathrm{L}$ & 0 & $\mathrm{GE}$ \\
\hline$\mu g / L$ & 0 & GE \\
\hline$\mu \mathrm{g} / \mathrm{L}$ & 0 & GE \\
\hline$\mu g / L$ & 0 & GE \\
\hline$\mu g / L$ & 0 & GE \\
\hline$\mu g / L$ & 0 & GE \\
\hline$\mu \mathrm{g} / \mathrm{L}$ & 0 & $\mathrm{GE}$ \\
\hline$\mu \mathrm{g} / \mathrm{L}$ & 0 & GE \\
\hline$\mu \mathrm{g} / \mathrm{L}$ & 0 & GE \\
\hline$\mu \mathrm{g} / \mathrm{L}$ & 0 & GE \\
\hline$\mu \mathrm{g} / \mathrm{L}$ & 0 & $\mathrm{GE}$ \\
\hline$\mu g / L$ & 0 & GE \\
\hline$\mu \mathrm{g} / \mathrm{L}$ & 0 & $\mathrm{GE}$ \\
\hline$\mu \mathrm{g} / \mathrm{L}$ & 0 & GE \\
\hline$\mu \mathrm{g} / \mathrm{L}$ & 0 & $\mathrm{GE}$ \\
\hline$\mu \mathrm{g} / \mathrm{L}$ & 0 & $\mathrm{GE}$ \\
\hline$\mu \mathrm{g} / \mathrm{L}$ & 0 & $\mathrm{GE}$ \\
\hline$\mu \mathrm{g} / \mathrm{L}$ & 0 & $\mathrm{GE}$ \\
\hline$\mu \mathrm{g} / \mathrm{L}$ & 0 & GE \\
\hline$\mu \mathrm{g} / \mathrm{L}$ & 0 & GE \\
\hline$\mu \mathrm{g} / \mathrm{L}$ & 0 & GE \\
\hline$\mu \mathrm{g} / \mathrm{L}$ & 0 & $\mathrm{GE}$ \\
\hline$\mu g / L$ & 0 & GE \\
\hline$\mu g / L$ & 0 & GE \\
\hline$\mu \mathrm{g} / \mathrm{L}$ & 0 & $\mathrm{GE}$ \\
\hline$\mu \mathrm{g} / \mathrm{L}$ & 0 & $\mathrm{GE}$ \\
\hline$\mu \mathrm{g} / \mathrm{L}$ & 0 & $\mathrm{GE}$ \\
\hline$\mu g / L$ & 0 & GE \\
\hline$\mu g / L$ & $?$ & GE \\
\hline$\mu \mathrm{g} / \mathrm{L}$ & 0 & GE \\
\hline$\mu \mathrm{g} / \mathrm{L}$ & 0 & $\mathrm{GE}$ \\
\hline$\mu \mathrm{g} / \mathrm{L}$ & 0 & GE \\
\hline$\mu \mathrm{g} / \mathrm{L}$ & 0 & GE \\
\hline$\mu \mathrm{g} / \mathrm{L}$ & 0 & $\mathrm{GE}$ \\
\hline$\mu g / L$ & 0 & $\mathrm{GE}$ \\
\hline$\mu \mathrm{g} / \mathrm{L}$ & 0 & GE \\
\hline$\mu \mathrm{g} / \mathrm{L}$ & 0 & $\mathrm{GE}$ \\
\hline$\mu g / L$ & 0 & $\mathrm{GE}$ \\
\hline$\mu \mathrm{g} / \mathrm{L}$ & 0 & GE \\
\hline$\mu \mathrm{g} / \mathrm{L}$ & 0 & $\mathrm{GE}$ \\
\hline$\mu \mathrm{g} / \mathrm{L}$ & 0 & $\mathrm{GE}$ \\
\hline$\mu g / L$ & 0 & $\mathrm{GE}$ \\
\hline$\mu \mathrm{g} / \mathrm{L}$ & 0 & $\mathrm{GE}$ \\
\hline$\mu \mathrm{g} / \mathrm{L}$ & 0 & GE \\
\hline$\mu g / \mathrm{L}$ & 0 & $\mathrm{GE}$ \\
\hline$\mu \mathrm{g} / \mathrm{L}$ & 0 & 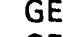 \\
\hline$\mu \mathrm{g} / \mathrm{L}$ & 0 & \\
\hline
\end{tabular}

= exceeded holding time.

= exceeded primary drinking water standard. 
WELL AMB 13AR collected on 05/08/92, laboratory anal, mes (cont.)

H D Analyte

Result

Unit $\quad$ Flag $\quad \underline{\text { Lab }}$

cis-1,3-Dichloropropene

trans-1,3-Dichloropropene

$<1.0$

Dieldrin

Diethyl phthalate

$<1.0$

$<0.50$

2,4-Dimeihyl phenol

$<10$

$<10$

Dimethyl phthalate

$<10$

$<45$

4-Dinitrophenol

$<10$

$<10$

2,6-Dinitrotoluene

$<10$

Ji-n-octyl phthalate

$<10$

2-Diphenylhydrazine

$<0.10$

Endosulfan I

$<0.10$

Endosulfan silfate

$<0.10$

Endrin

Endrin aldehyde

$<0.0060$

Ethylbenzene

$<0.10$

Fluoranthene

Fluorene

Fluoride

Fluoride

Heptachlor

Heptachlor epoxide

Hexachlorobenzene

Hexachlorobutadiene

$<1.0$

$<10$

$<10$

$<100$

$<100$

$<0.050$

$<0.050$

$<10$

Hexachlorocyclopentadiene

$<10$

$<10$

Hexachloroethane

$<10$

Indenol 1,2,3-c, olpyrene

Iron

Iron

Isophorone

Lead

Lindane

Magnesium

Magnesium

Manganese

Manganese

Mercury

2-Methyl-4,6-dinitrophenol

$<10$

54

54

$<10$

$<3.0$

$<0.0050$

430

435

14

14

$<0.20$

Naphthalene

$<10$

$<10$

Nickel

Nickel

ivitrate as nitrogen

$<4.0$

$<4.0$

680

$<10$

2-Nitrophenol

4-Nitrophenol

$<10$

$<10$

$<10$

$<10$

$<10$

$<0.50$

$\mathrm{N}$-Nitrosodipropylamine

$<0.50$

PCB 1221

$<0.50$

$<0.50$

$<0.50$

PCB 1248

PB 1254

$<0.50$

$\mu g / L \quad O \quad$ GE

$\mu g / L \quad 0 \quad G E$

$\mu g / L \quad 0 \quad G E$

$\mu g / L \quad 0 \quad$ GE

$\mu g / L \quad 0 \quad G E$

$\mu g / L \quad 0 \quad$ GE

$\mu g / L \quad 0 \quad G E$

$\mu g / L \quad O \quad G E$

$\mu \mathrm{g} / \mathrm{L} \quad \mathrm{O} \quad \mathrm{GE}$

$\mu g / L \quad 0 \quad$ GE

$\mu g / L \quad 0 \quad$ GE

$\mu g / L \quad 0 \quad G E$

$\mu g / L \quad 0 \quad$ GE

$\mu g / L \quad 0 \quad G E$

$\mu g / L \quad 0 \quad G E$

$\mu g / L \quad 0 \quad$ GE

$\mu g / L \quad O \quad$ GE

$\mu g / L \quad 0 \quad G E$

$\mu g / L \quad O \quad$ GE

$\mu g / L \quad 0 \quad$ GE

$\mu g / L \quad O \quad G E$

$\mu g / L \quad 0 \quad$ GE

$\mu \mathrm{g} / \mathrm{L} \quad \mathrm{O} \quad \mathrm{GE}$

$\mu g / L \quad 0 \quad G E$

$\mu g / L \quad 0 \quad G E$

$\mu g / L \quad 0 \quad$ GE

$\mu g / L \quad 0 \quad G E$

$\mu g / L \quad O \quad G E$

$\mu \mathrm{g} / \mathrm{L} \quad \mathrm{O} \quad \mathrm{GE}$

$\mu g / L \quad O \quad G E$

$\mu g / L \quad 0 \quad G E$

$\mu g / L \quad 0 \quad G E$

$\mu g / L \quad 0 \quad$ GE

$\mu g_{i} . \quad 0 \quad$ GE

$\mu \mathrm{g} / \mathrm{L} \quad \mathrm{O} \quad \mathrm{GE}$

$\mu g / L \quad 0 \quad$ GE

$\mu g / L \quad 0 \quad$ GE

$\mu g / L \quad 0 \quad$ GE

$\mu \mathrm{g} / \mathrm{L} \quad \mathrm{O} \quad \mathrm{GE}$

$\mu g / L \quad 0 \quad G E$

$\mu \mathrm{g} / \mathrm{L} \quad \mathrm{O} \quad \mathrm{GE}$

$\mu g / L \quad 0 \quad$ GE

$\mu g / L \quad 0 \quad G E$

$\mu \mathrm{g} / \mathrm{L} \quad 0 \quad \mathrm{GE}$

$\mu g / L \quad 0 \quad$ GE

$\mu g / L \quad 0 \quad$ GE

$\mu g / L \quad 0 \quad G E$

$\mu \mathrm{g} / \mathrm{L} \quad \mathrm{O} \quad \mathrm{GE}$

$\mu g / L \quad 0 \quad G E$

$\mu g / L \quad 0 \quad$ GE

$\mu g / L \quad 0 \quad G E$

$\mu \mathrm{g} / \mathrm{L} \quad \mathrm{O} \quad \mathrm{GE}$

$\mu \mathrm{g} / \mathrm{h} \quad \mathrm{O} \quad \mathrm{GE}$

$\mu \mathrm{g} / \mathrm{L} \quad \mathrm{O} \quad \mathrm{GE}$

$\mu g / L \quad O \quad G E$

PCB 1260

$\mu \mathrm{g} / \mathrm{L}$

GE

- exceeded holding time. = exceeded primary drinking water stanciard. 
WELL AMB 13AR collected on 05/08/92, laboratory analyses (cont.)

H D Analvte

Pentachlorophenol

Phenanthrene

Phenol

Phenols

Potassium

Potassium

Pyrene

Selenium

Silica

Silica

Silver

Silver

Sodium

Sodium

Sulfate

1,1,2,2-Tetrachloroethane

Tetrachloroethylene

Thallium

Toluene

Total dissolved solids

Total organic carbon

Total organic halogens

Total phosphates (as P)

Toxaphene

1,2,4-Trichlorobenzene

1,1,1-Trichloroethane

1,1,2-Trichloroethane

Trichloroethylene

Trichlorofluoromethane

2,4,6-Trichlorophenol

Zinc

Zinc

Gross alpha

Nonvolatile beta

Total alpha-emitting radium

Total alpha-emitting radium

Tritium

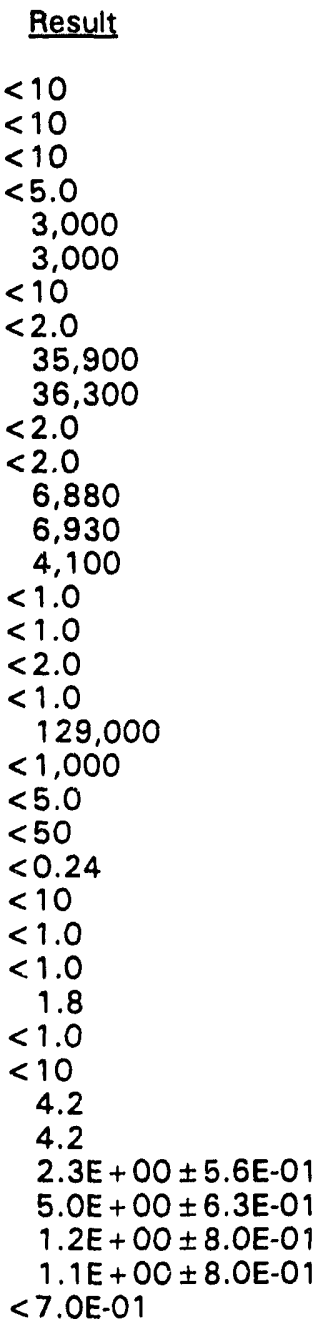

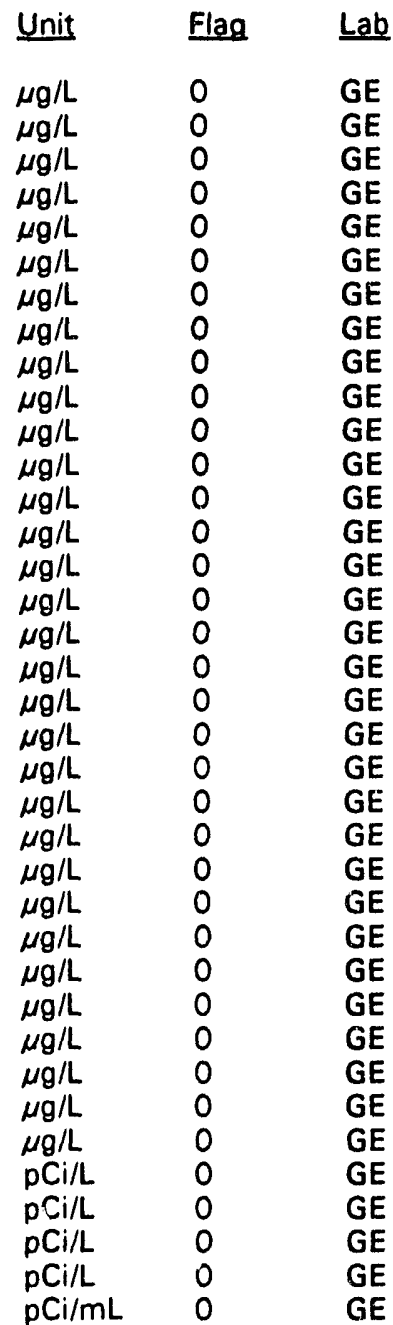

C

C

- =xceeded holding timc. $=$ - exceeded primary drinking water standard. 


\section{Appendix E - Time Series Plots}




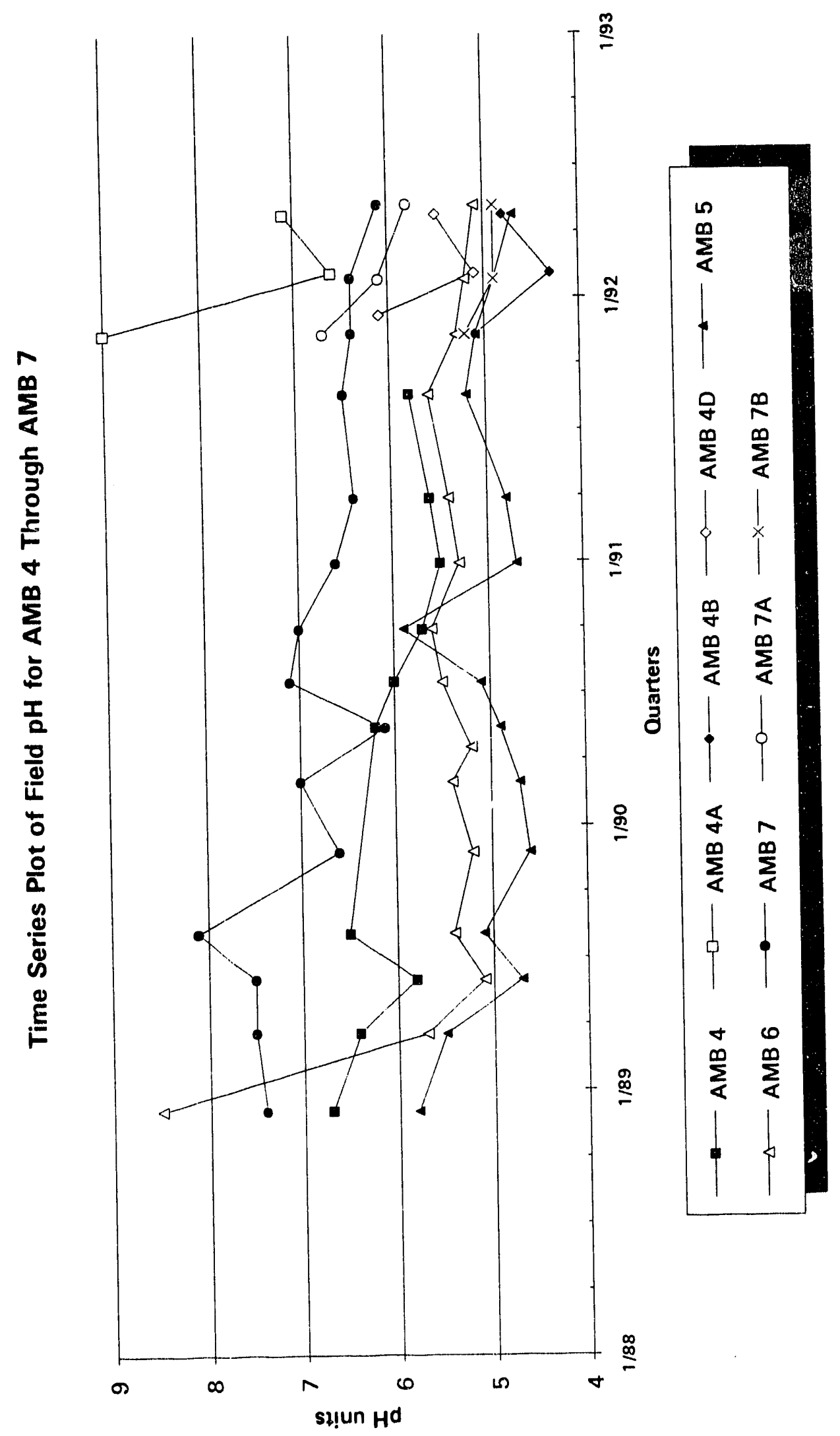

c

e

$c$

C

? 

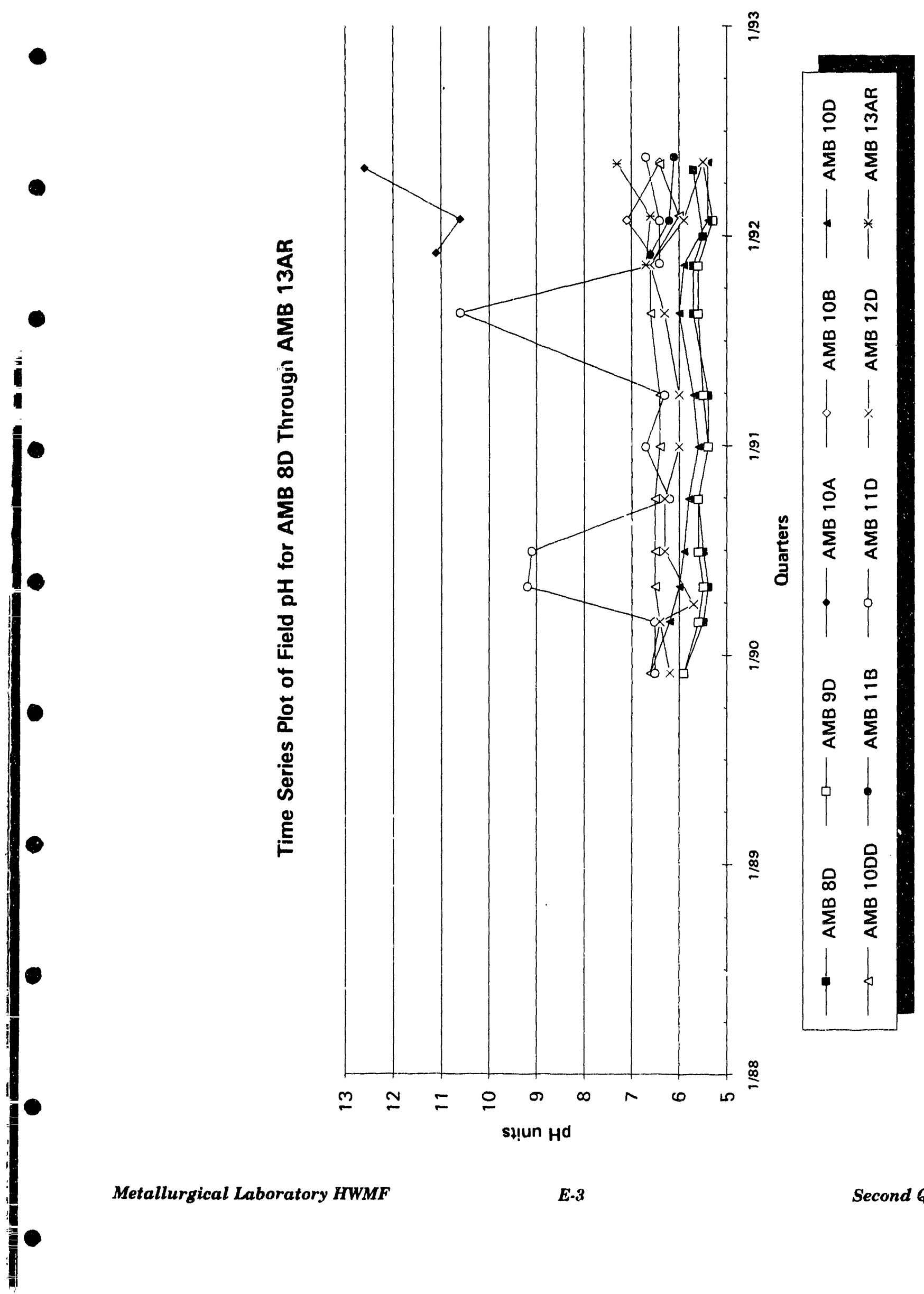


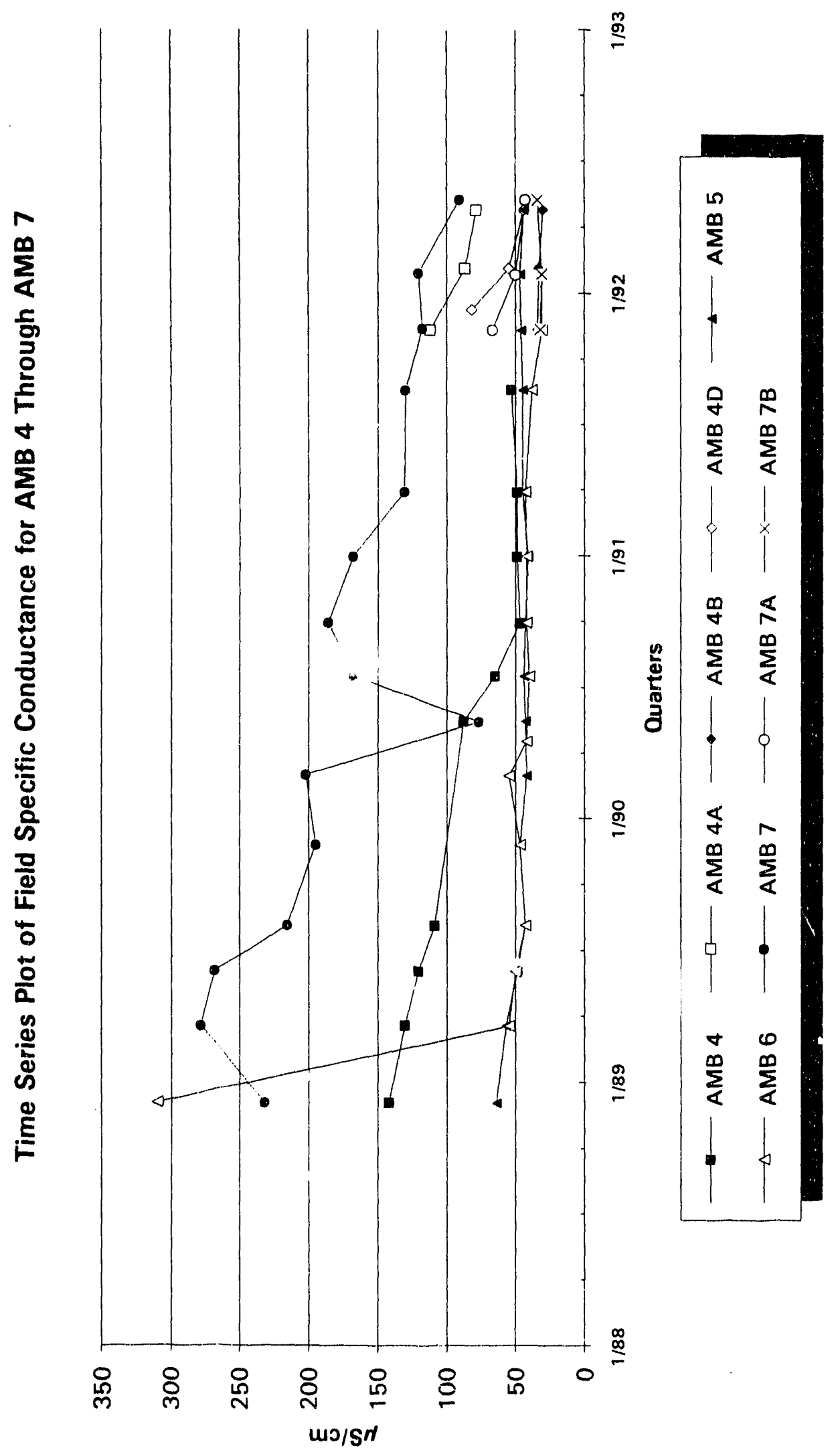

8

c 


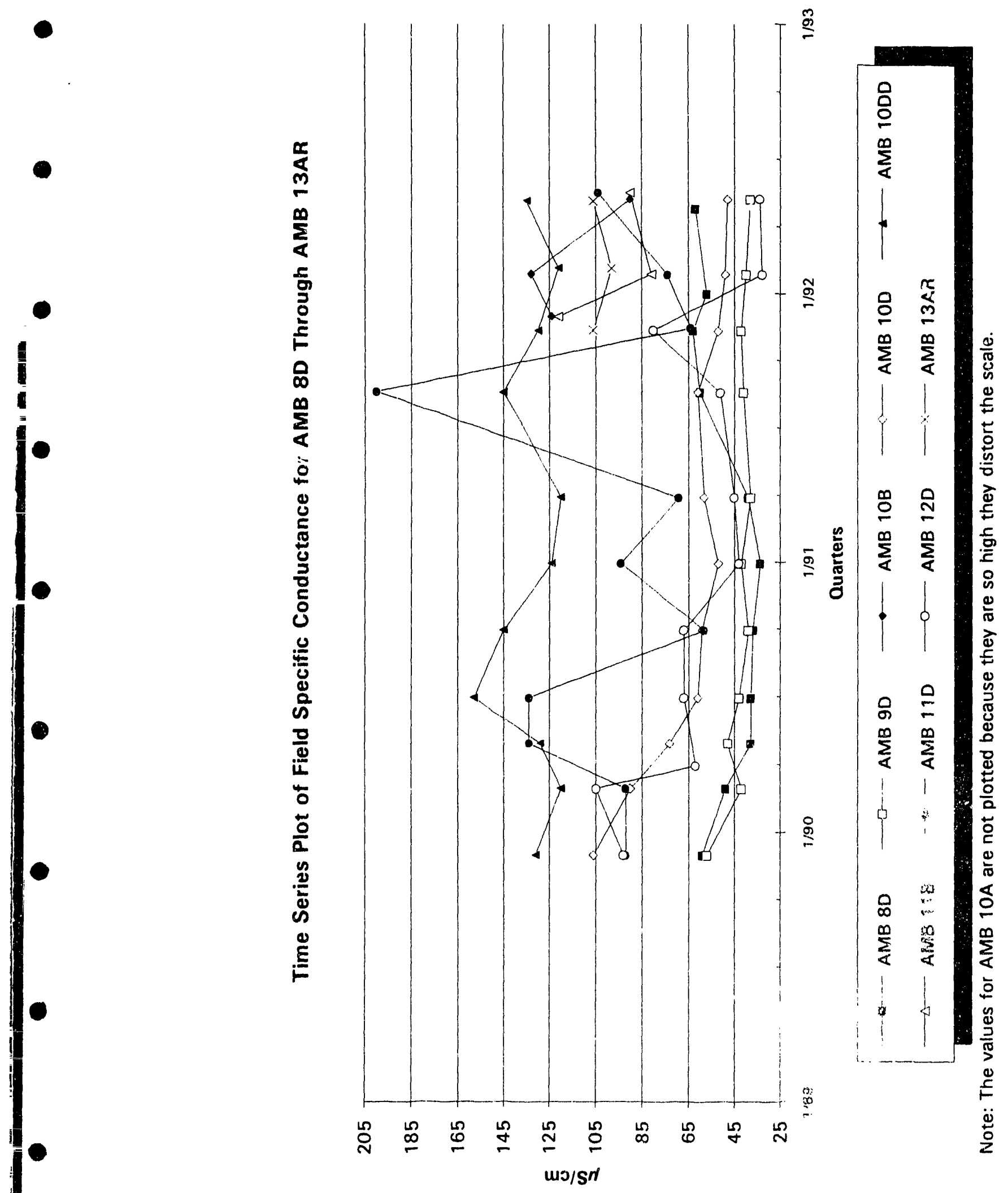




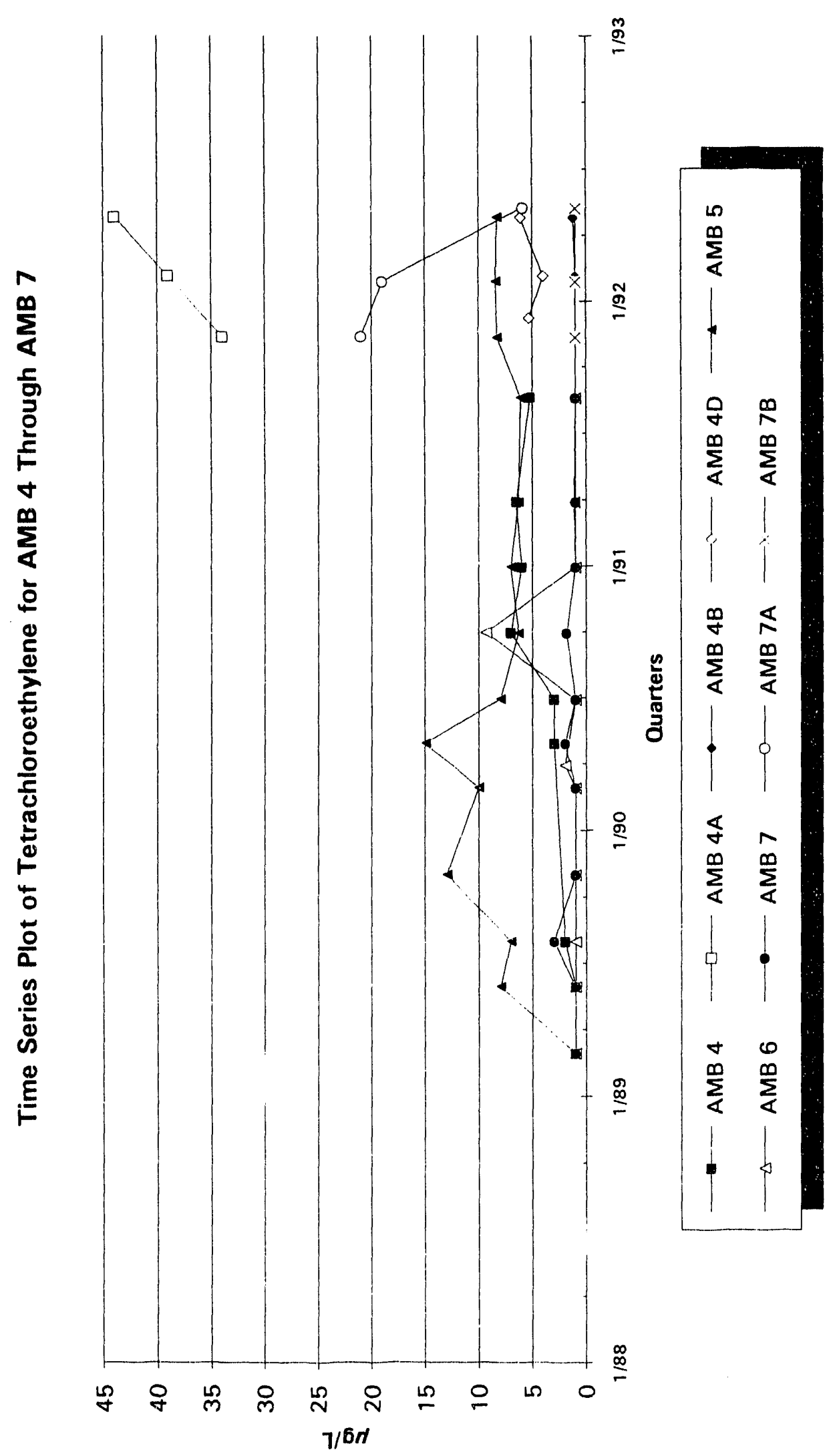

c

1

Second Quarter 1992 


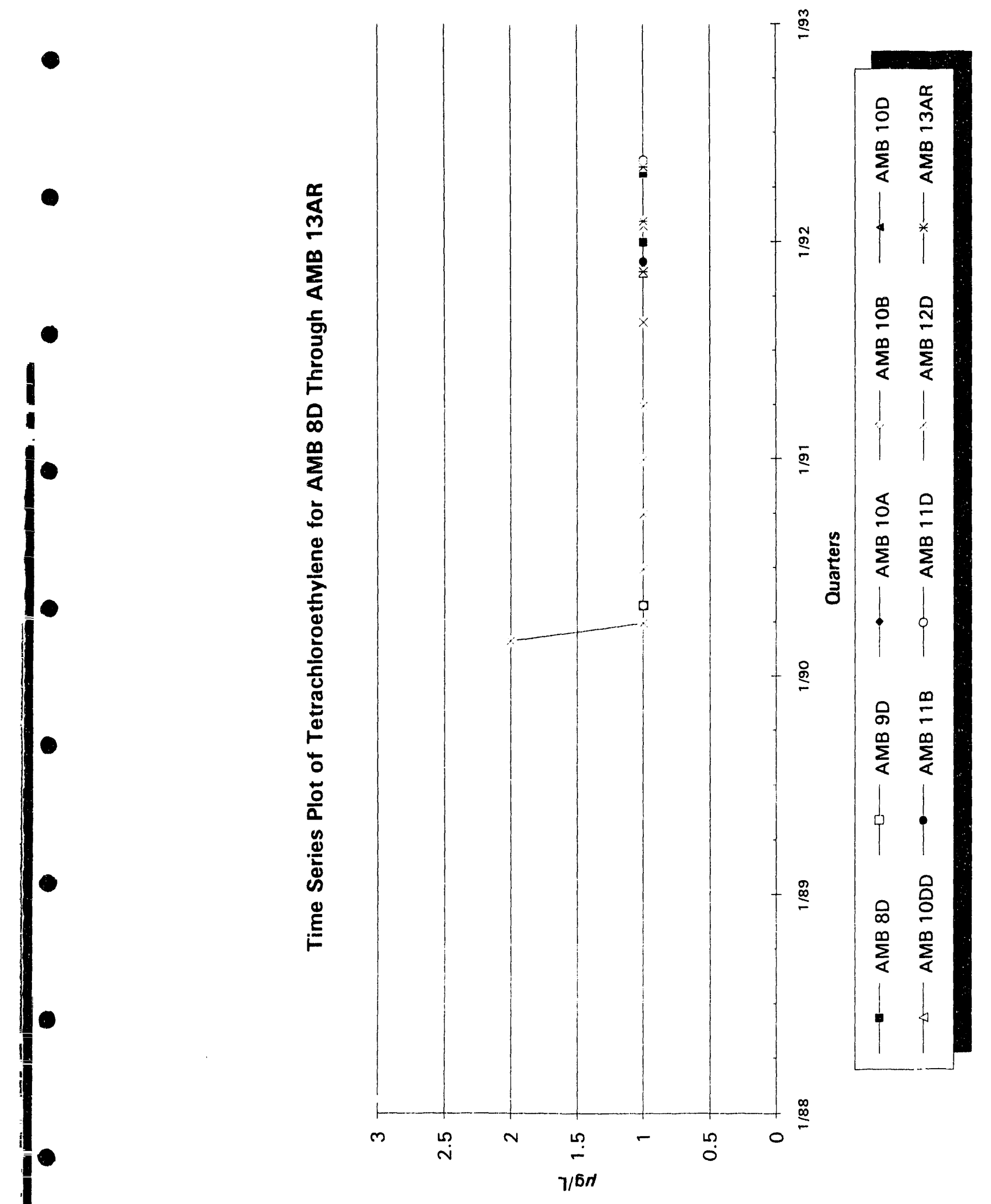




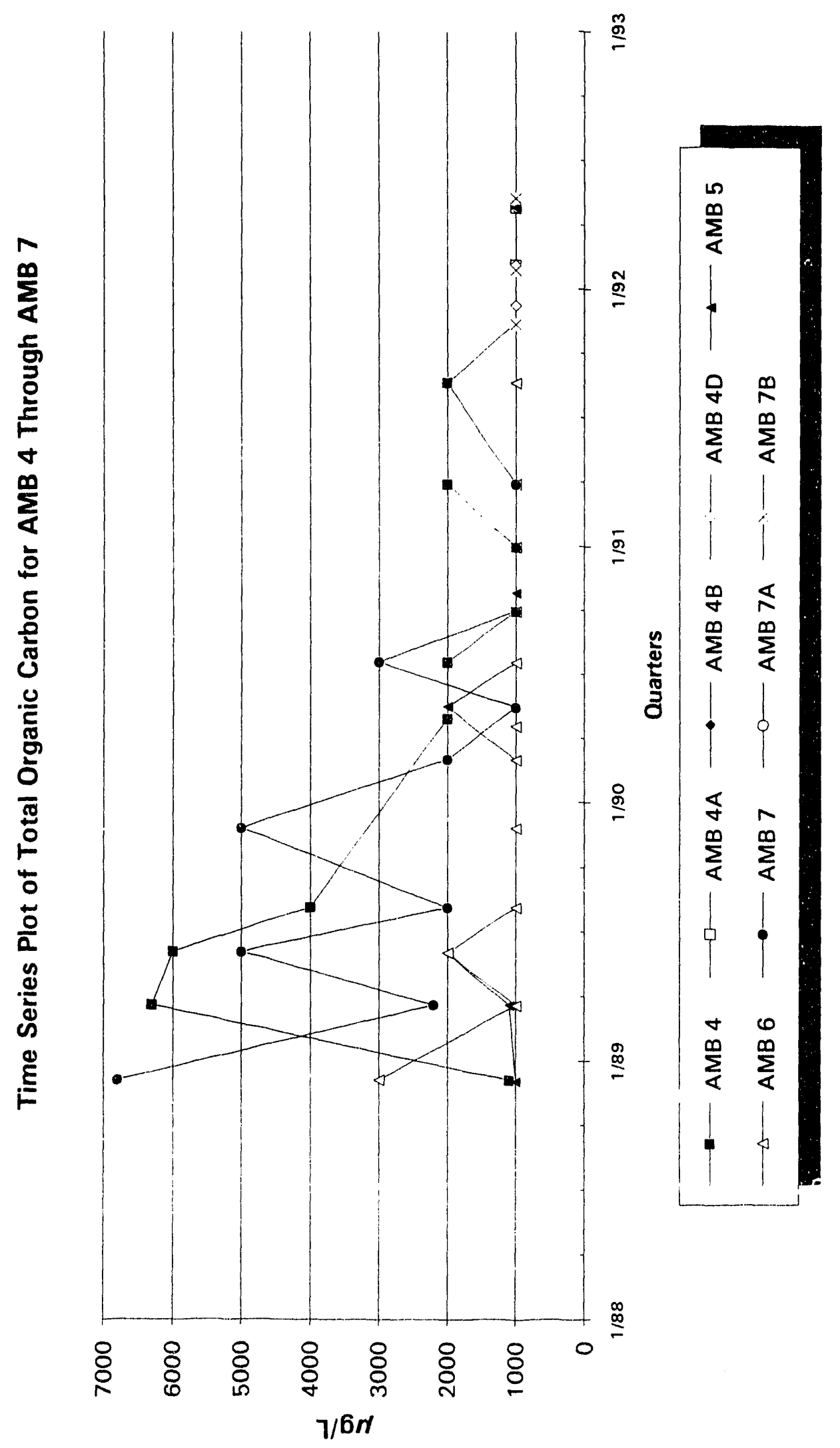

$c$

E

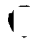

Second Quarter 1992 


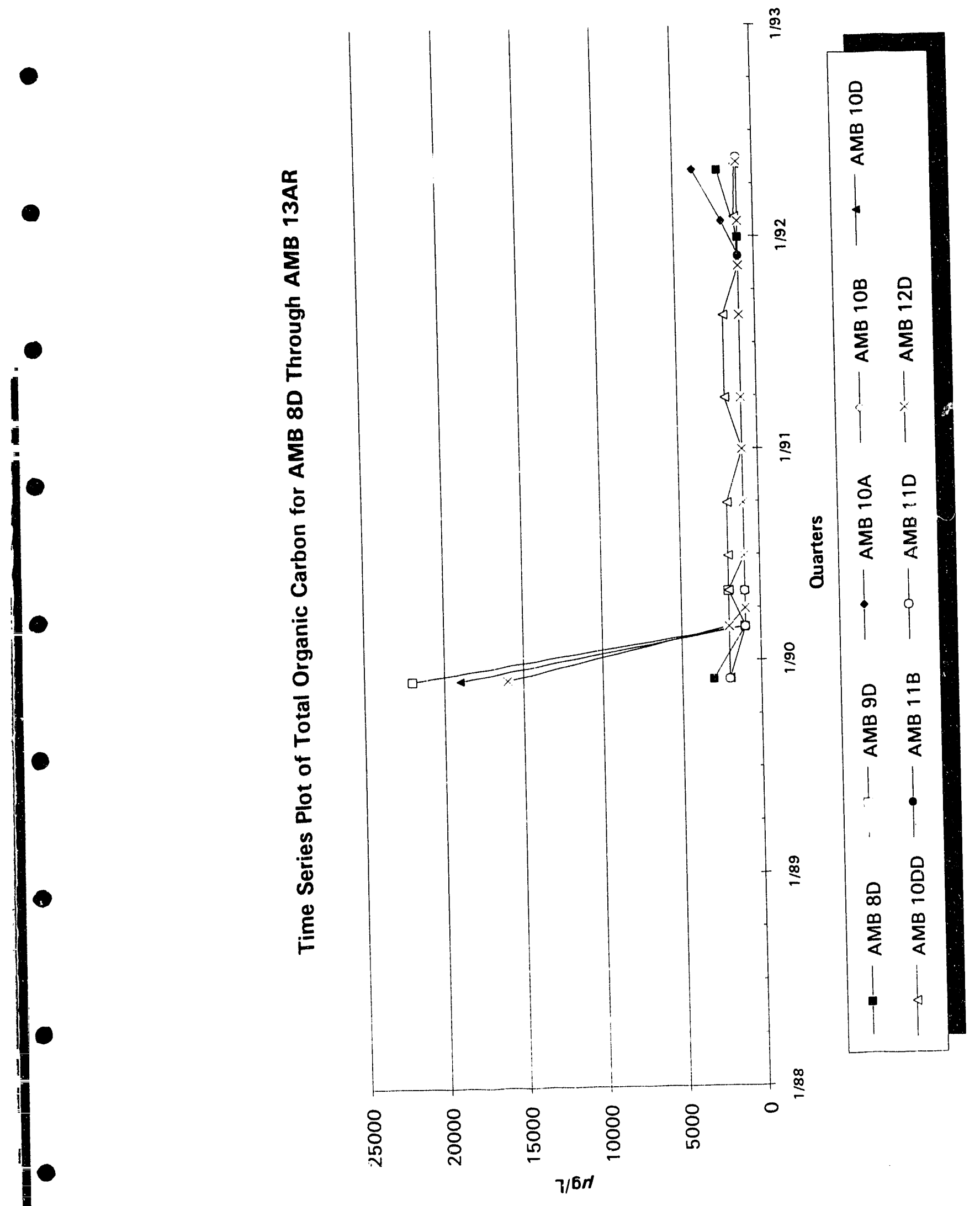




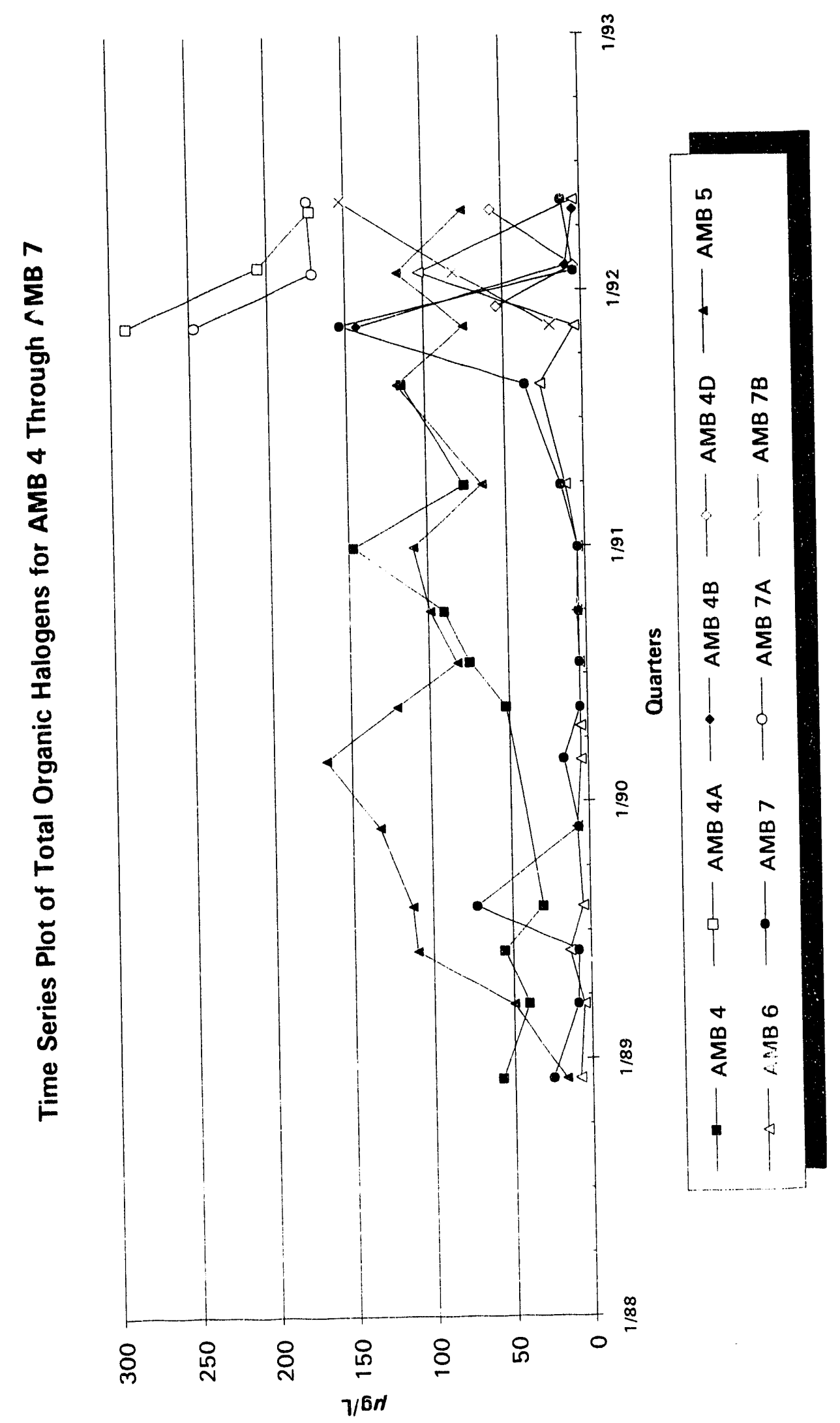

-

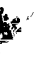



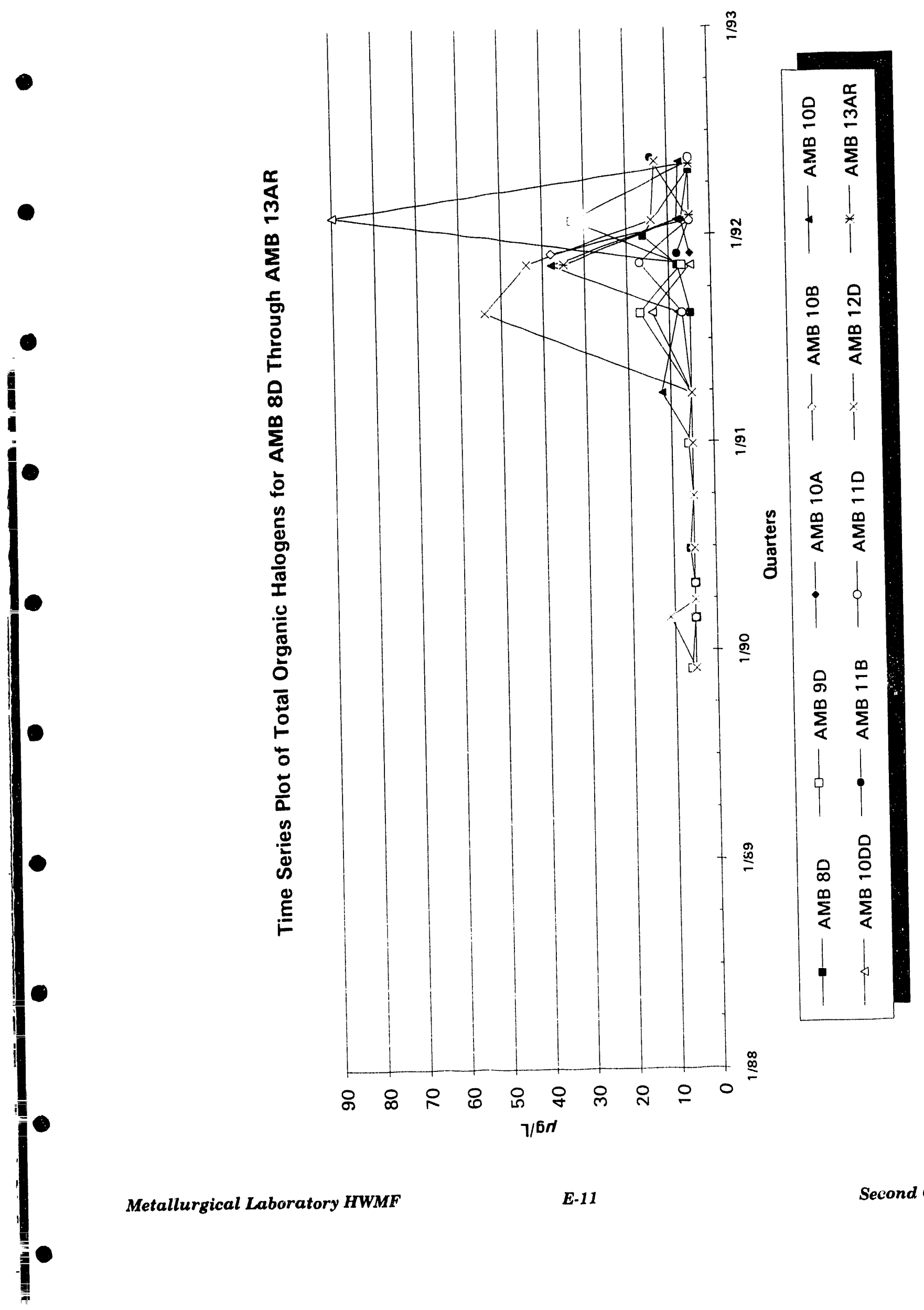


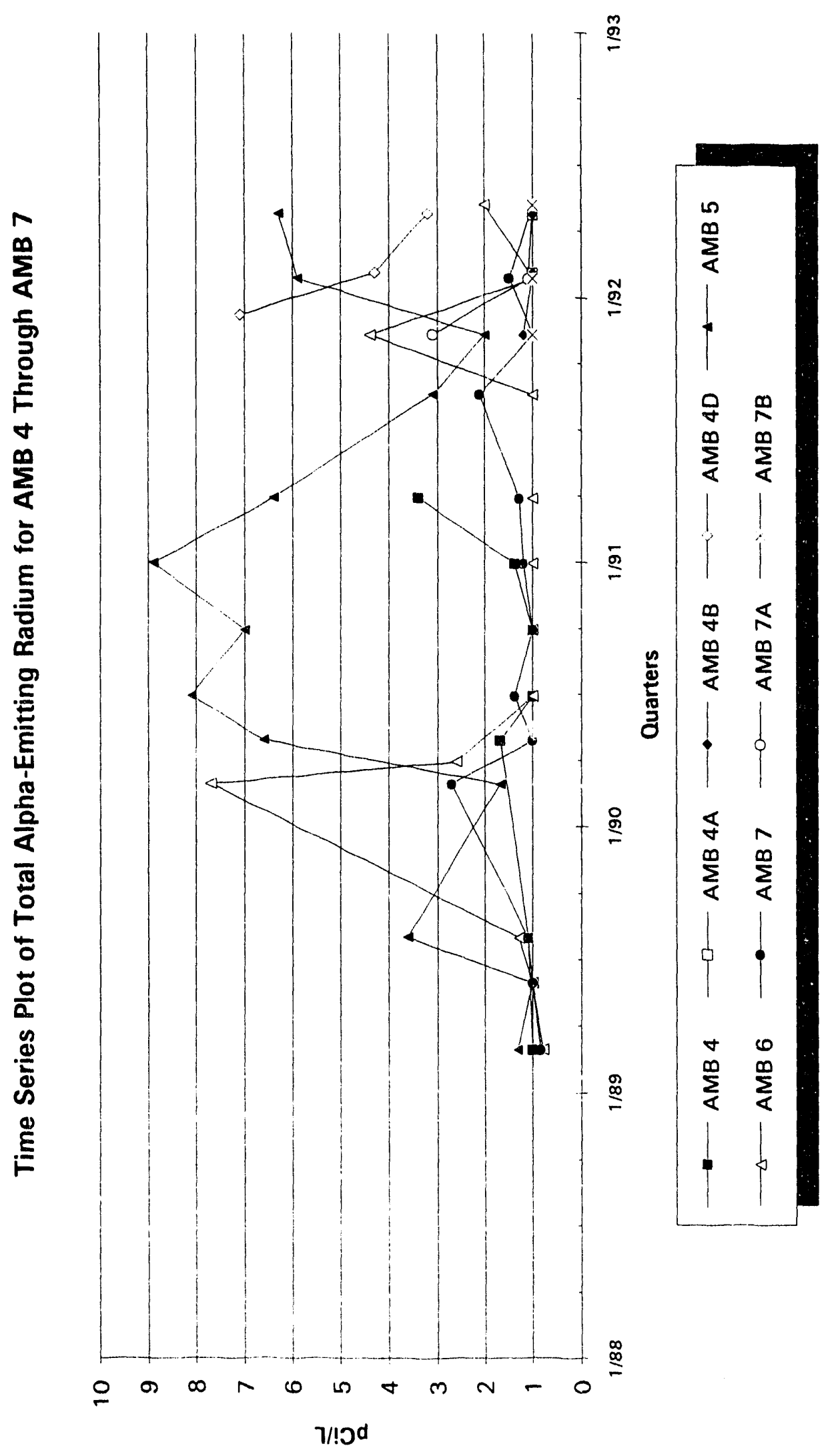

e

$c$

C

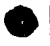

- 


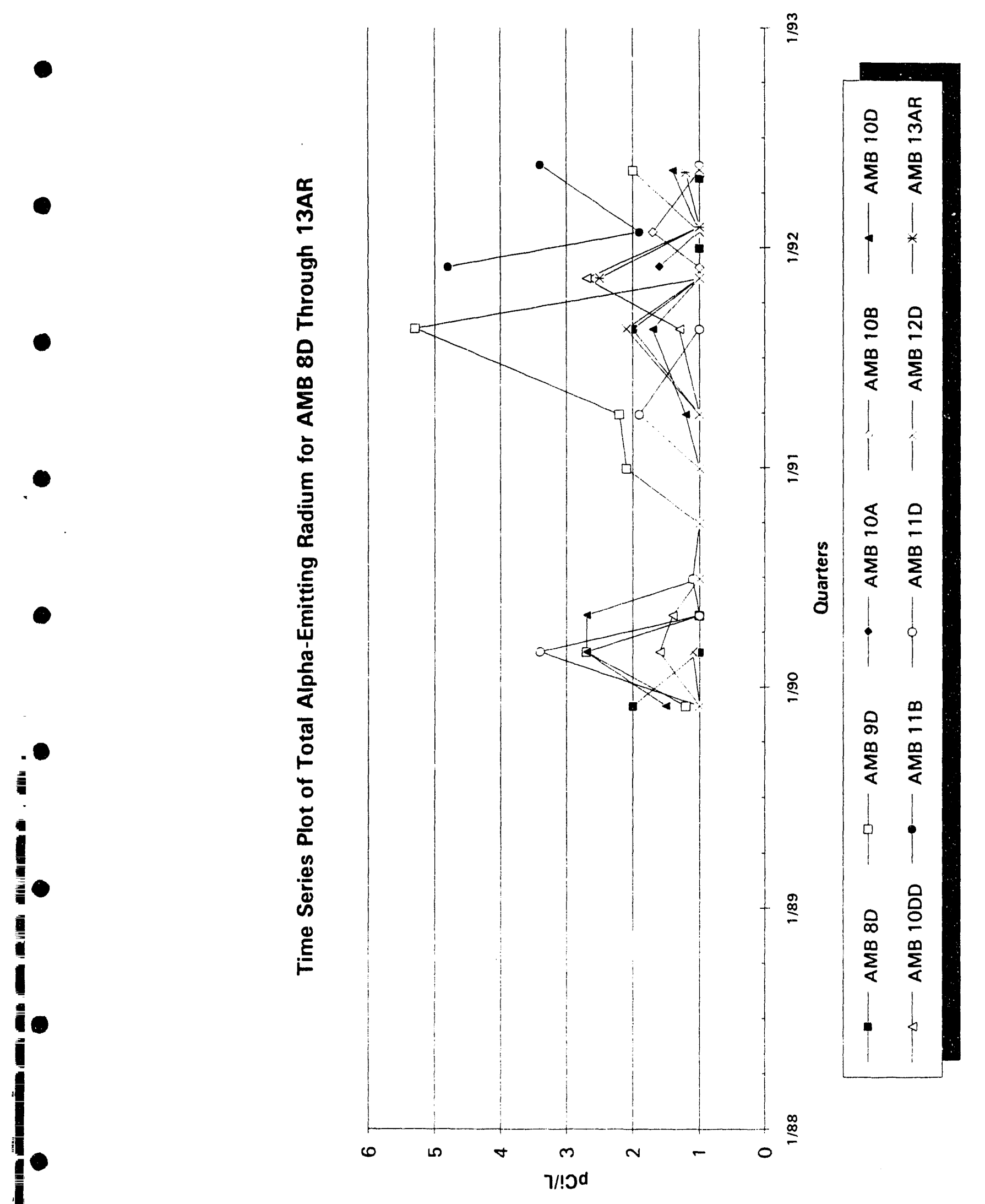




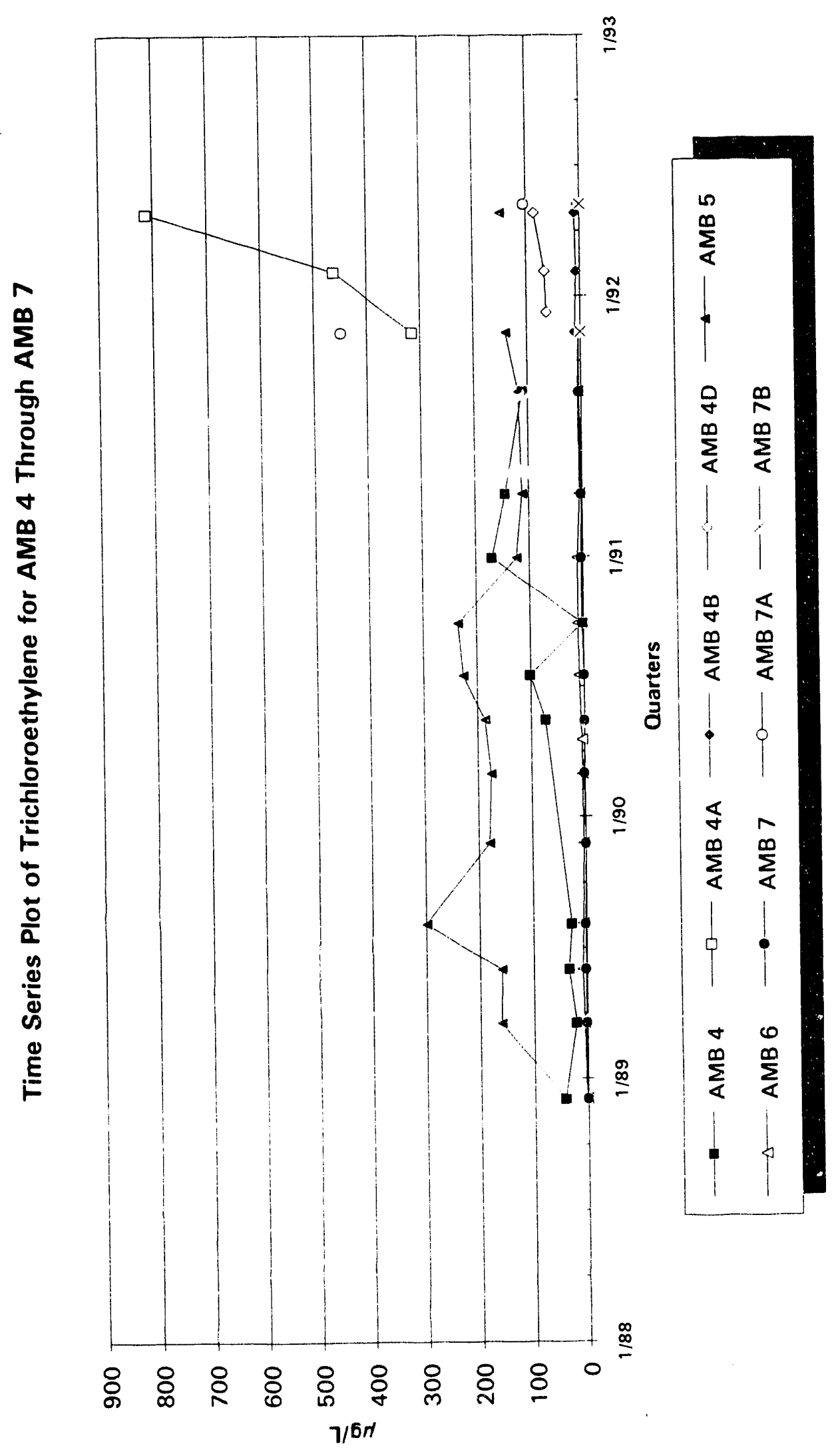

○ 


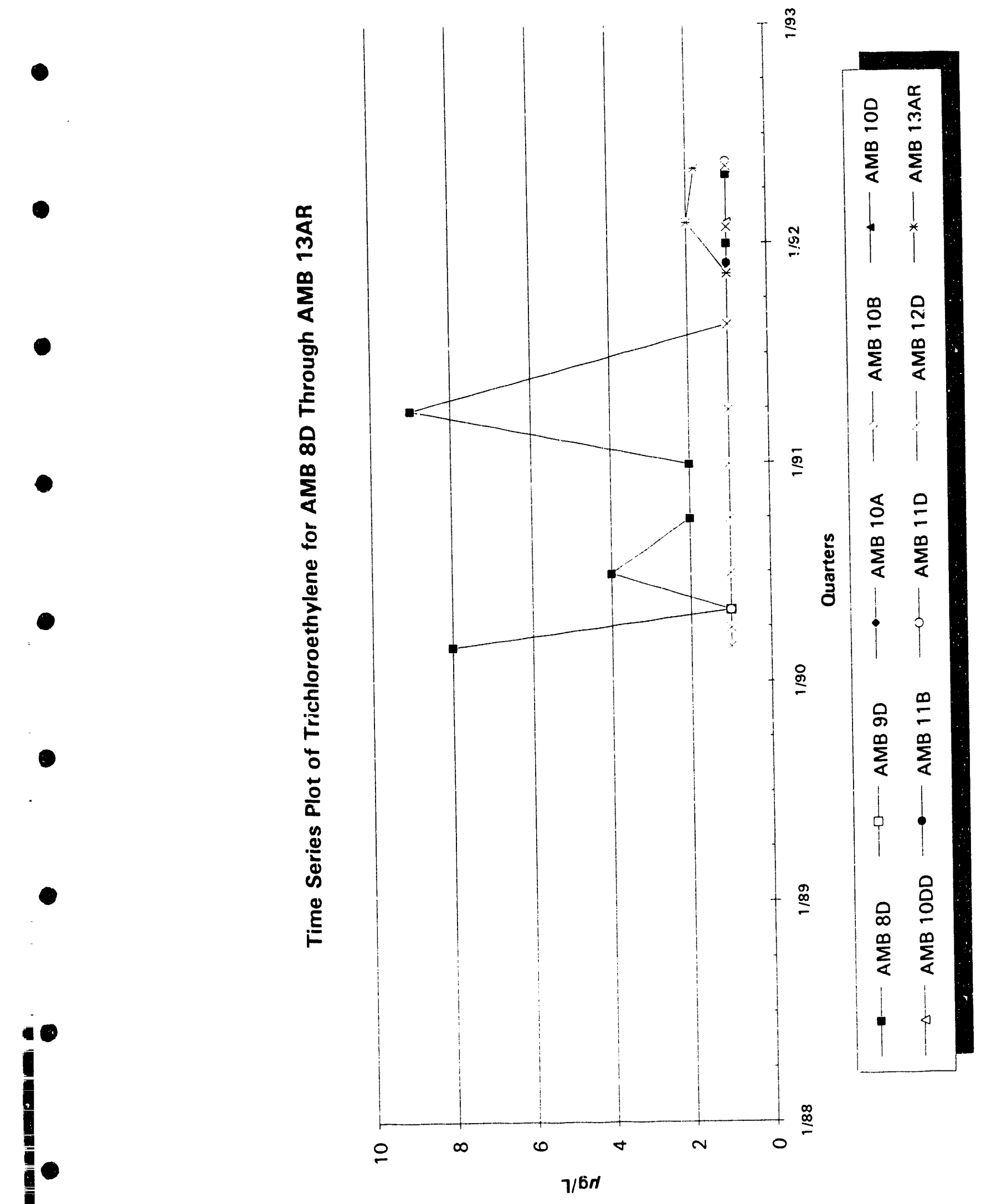




\section{Appendix F - Hydrographs}

0

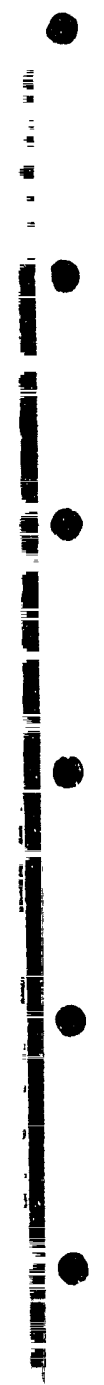



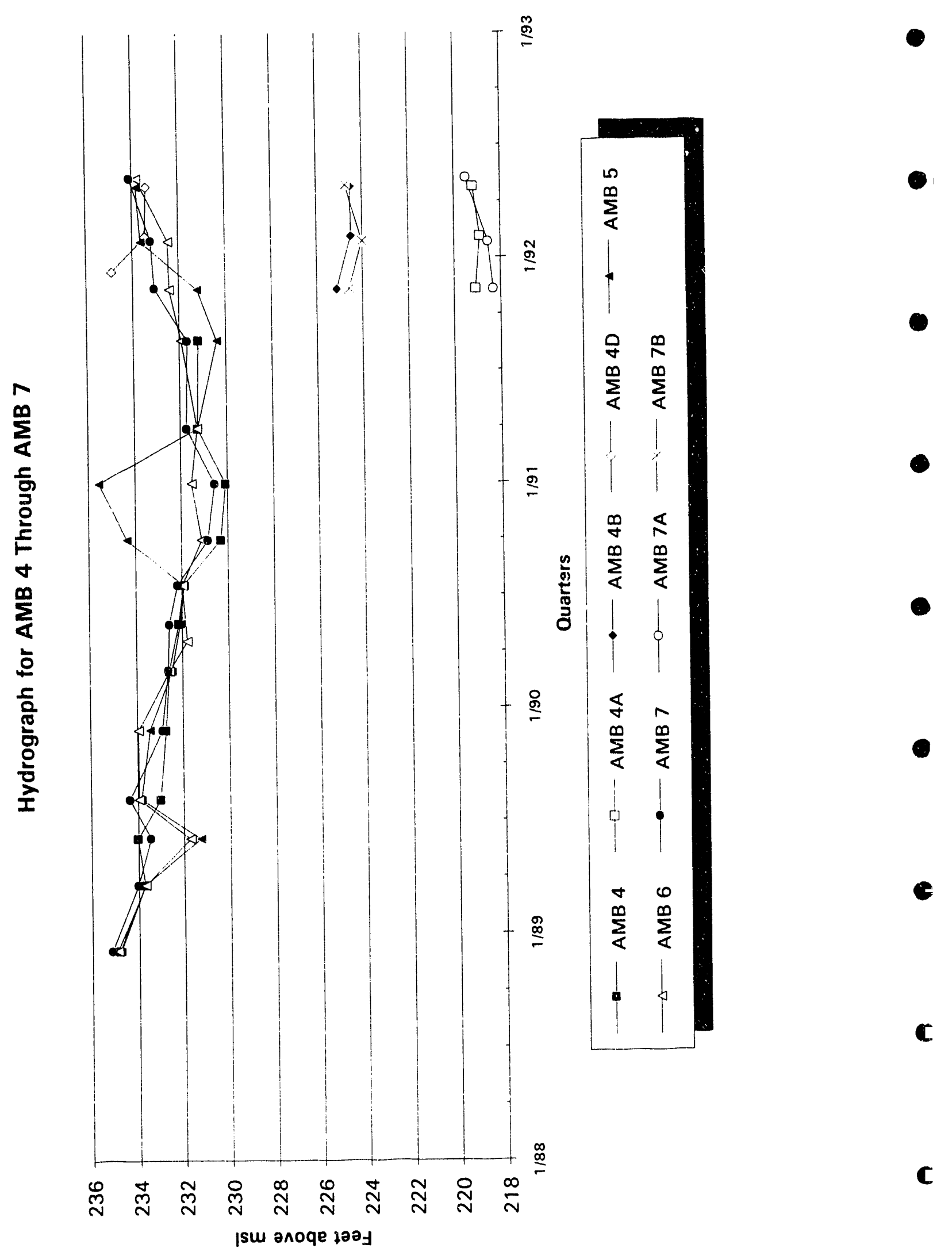


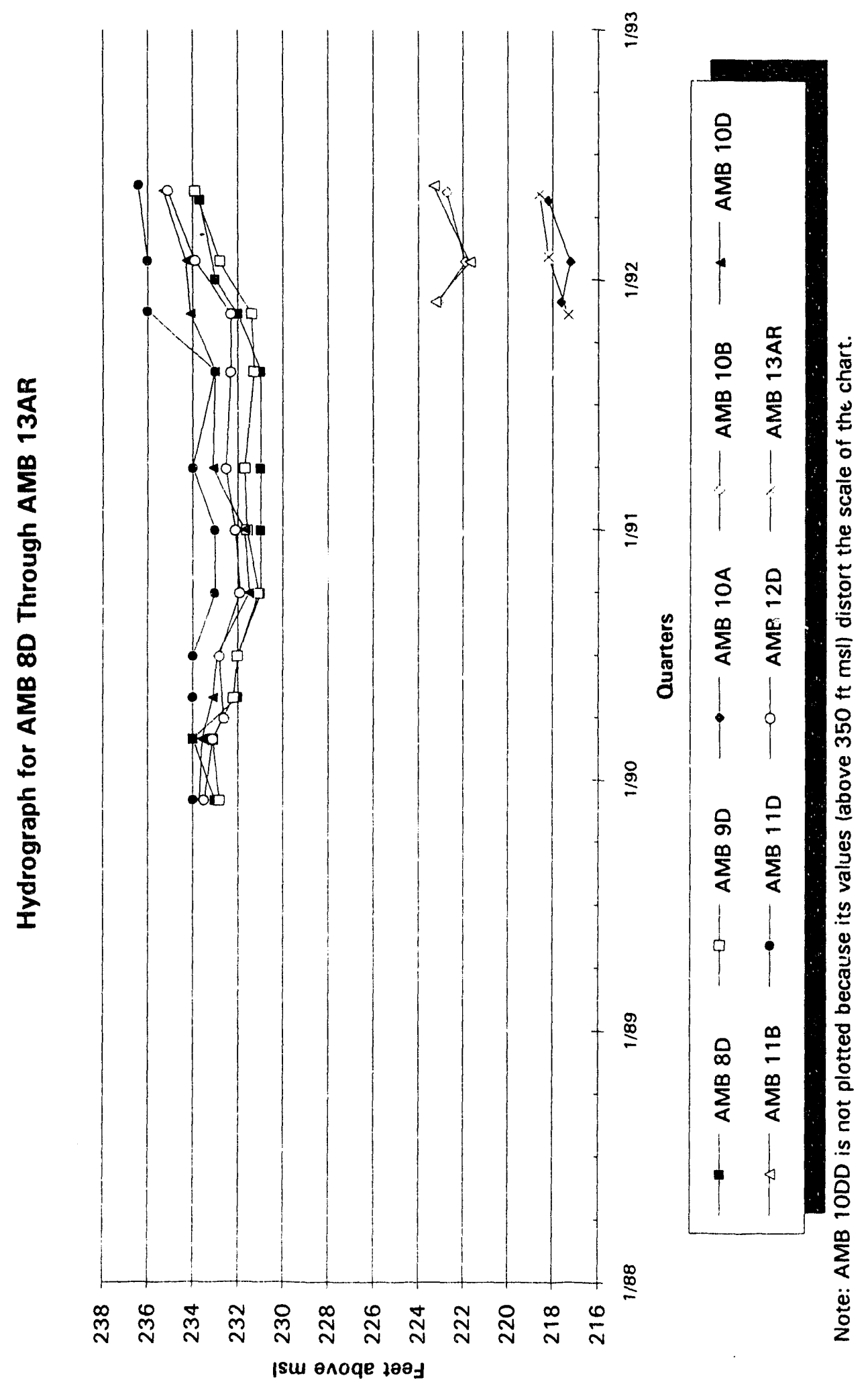


E

E

\author{
.
}


Map 1. Water-Hlevation Contour Map of the Water-Iable Unit at the M-Area Hazardoun Waste Management Facility, Second Quarter l\$y! 
10

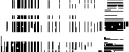

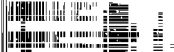

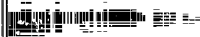

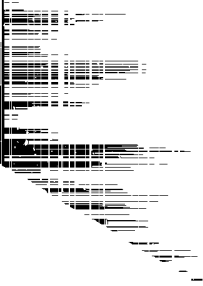

Map 2. Potentiometric Surface Map of the L'pper Seceion of the congaree L'nit at the M.Area Hazardous Waste Management Facility. Siecond Quarter 1992 


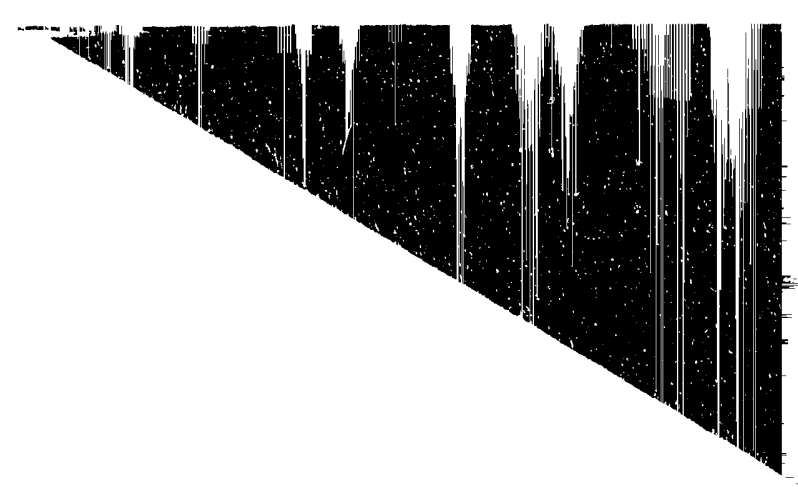

$1 M$

'

4

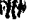

称

Map 3. Potentiometric Surface Map of the Lower Section of the Congaree Unit at the M-Area Hazardous Waste Management Facility. Second Quarter 1992 

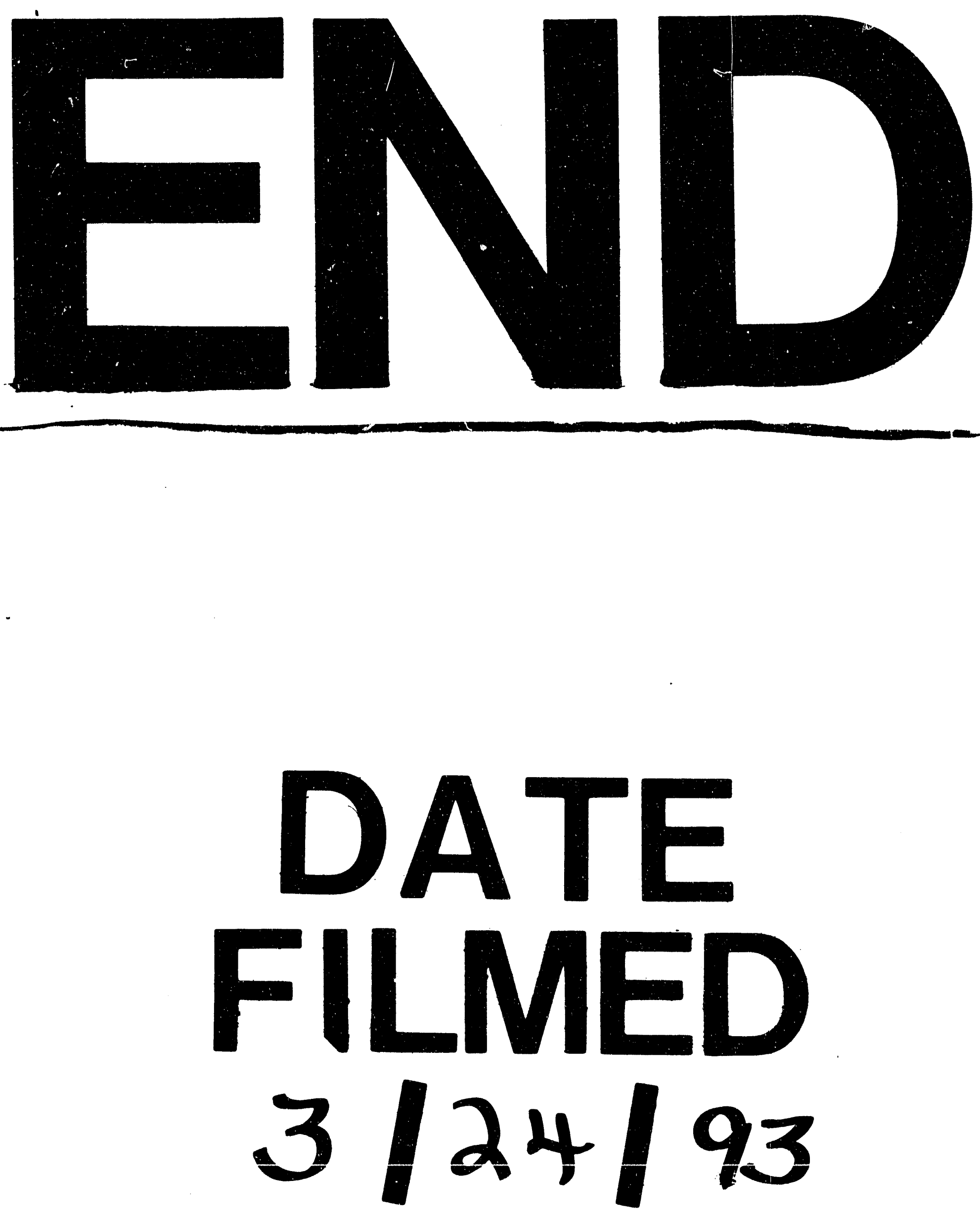
